\title{
FIRST-PRINCIPLES INVESTIGATION OF TRANSPORT AND MAGNETISM
}

\author{
A Thesis presented to \\ the Faculty of the Graduate School \\ at the University of Missouri
}

In Partial Fulfillment

of the Requirements for the Degree

Doctor of Philosophy

\author{
by \\ Aditya Putatunda
}

Dr. David J Singh, Thesis Supervisor

MAY 2021 
The undersigned, appointed by the Dean of the Graduate School, have examined the dissertation entitled:

\section{FIRST-PRINCIPLES INVESTIGATION OF TRANSPORT AND MAGNETISM}

presented by Aditya Putatunda, a candidate for the degree of Doctor of Philosophy and hereby certify that, in their opinion, it is worthy of acceptance.

\begin{tabular}{c}
\hline Dr. David J Singh \\
\hline Dr. Guang Bian \\
\hline Dr. Wouter Montfrooij \\
Dr. Jian Lin
\end{tabular}




\section{ACKNOWLEDGMENTS}

I wish to acknowledge my deep gratitude towards my thesis advisor Prof. David Singh. It was his kindness that I was given the opportunity to work with him and his patience and support that lead me to finish this dissertation. He has always been available to help me with my academic challenges while also motivating me during my personal issues. I also received his financial support which enabled me to concentrate on my research. I am utmost grateful for his wise but simple advices which I am sure has shaped my personality for better. I am hopeful his continuous attention towards improving my scientific communication skills be it publishing my research or to develop my presentation skills, has helped me grow into a stronger researcher. He suggested me research topics that were deliberately chosen to suit my abilities while slowly pushing me up the learning curve. His patience and sincere care towards my progress lead me to continue my journey, all of which finally resulted into this thesis.

While working with Prof. Singh, I was also fortunate to be among my co-workers. I specially wish to thank Jifeng Sun, whose help with my early introduction to research and computational techniques I deeply appreciate. I am also grateful towards Guangzong Xing, Yuwei Li for both of their professional and personal help. Their work with the electronic fitness function and useful discussions on this topic greatly benefited me. I am also thankful to Zhenzhen Feng whose dedication and intelligent approach towards her research motivated me. I am grateful to have assisted her with her study of half-Heusler thermoelectric materials. I wish to convey my thanks to Guanhua Qin with whom I worked on the strontium ruthenate material and BingHua Lei for his useful discussions. I also acknowledge the contributions of Matt and 
Cory which lead to the project on selenites.

I also very much admire my friendship with Tuhin, Tonmoy, Raoul and Pranshu whose companionship made my stay at Columbia enjoyable and helped me cope with personal challenges. I thank the staff of Department of Physics and Astronomy and the University of Missouri whose timely assistance made me feel welcome and pleasant and helped me manage my official matters promptly. I deeply thankful towards my thesis committee members for their valuable time and for considering to examine my thesis.

Lastly, I thank my parents and my siblings who supported me throughout my journey as a graduate student and motivated me to push through all the challenges I faced during these last five years. This dissertation wouldn't have been possible without their love. 


\section{TABLE OF CONTENTS}

ACKNOWLEDGMENTS ................ ii

LIST OF TABLES $\ldots \ldots \ldots \ldots \ldots \ldots$ vii

LIST OF FIGURES $\ldots \ldots \ldots \ldots \ldots \ldots \ldots \ldots \ldots \ldots \ldots \ldots \ldots$

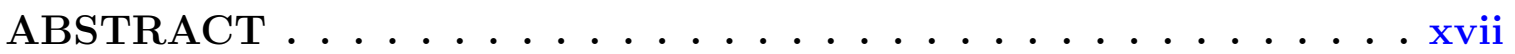

\section{CHAPTER}

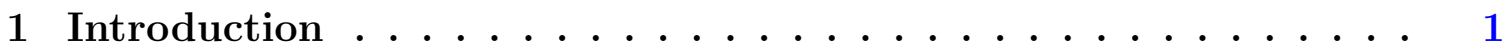

2 Methods and theoretical background $\ldots \ldots \ldots \ldots$

2.1 Addressing the many body challenge $\ldots \ldots \ldots \ldots \ldots$

2.1.1 Antisymmetric nature of the electron wavefunction: . . . . . 9

2.2 Density functional theory . . . . . . . . . . . . . . . . 10

2.2.1 The Hohenberg-Kohn theorems: . . . . . . . . . . . . . . . 11

2.3 The Kohn-Sham formalism: . . . . . . . . . . . . . . . . . . . . 14

2.3.1 The exchange-correlation term: . . . . . . . . . . . 17

2.3 .2 The choice of basis . . . . . . . . . . . . . . . 21

2.4 Brief exposition of the explored phenomena $\ldots \ldots \ldots \ldots$

2.4 .1 Thermoelectricity . . . . . . . . . . . . . . . 24

2.4 .2 Magnetism. . . . . . . . . . . . . . . . . . . . 28

3 Demonstration of first-principles tools: Case studies of two different materials . . . . . . . . . . . . . . 32

3.1 Ground state energy ordering of $\mathrm{TiO}_{2}$ polymorphs $\ldots \ldots \ldots \ldots$ 
3.1 .1 Introduction . . . . . . . . . . . . . . . 34

3.1 .2 Computational details . . . . . . . . . . . . . 37

3.1 .3 Results and discussion $\ldots \ldots \ldots \ldots$

3.1 .4 Conclusions . . . . . . . . . . . . . . . . . . . . 39

$3.2 \mathrm{NaSbSe}_{2}$ : A study of its thermoelectric properties . . . . . . . . 42

3.2 .1 Introduction . . . . . . . . . . . . . . . . 42

3.2 .2 Computational details . . . . . . . . . . . . . . . . 43

3.2 .3 Results and discussion . . . . . . . . . . . . . . . 44

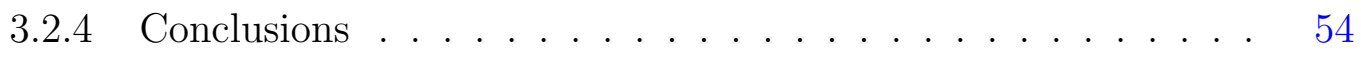

4 Thermoelectrics: An insight into the Lorenz number . . . . . . 56

4.1 Introduction $\ldots \ldots \ldots \ldots \ldots \ldots \ldots \ldots \ldots \ldots \ldots$

4.2 Computational methods . . . . . . . . . . . . . . 62

4.3 Results and discussion . . . . . . . . . . . . . . . . 64

4.4 Conclusions . . . . . . . . . . . . . . . . . . . . 71

5 Magnetism: a case study of its localized type $\ldots \ldots \ldots \ldots 72$

5.1 Properties of the antiferromagnetic selenite: $\mathrm{MnSeO}_{3}$ vs $\mathrm{ZnSeO}_{3} \ldots 73$

5.2 Introduction . . . . . . . . . . . . . . . . . 74

5.3 Computational approach . . . . . . . . . . . . . . 75

5.4 Results and discussion $\ldots \ldots \ldots \ldots \ldots \ldots$

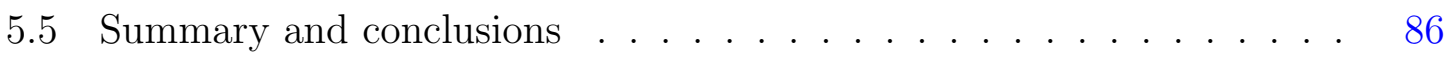

6 Quantum criticality and the search for competing magnetic orders in $\mathrm{Sr}_{3} \mathrm{Ru}_{2} \mathrm{O}_{7} \ldots \ldots \ldots \ldots \ldots \ldots \ldots \ldots$

6.1 Quantum critical point . . . . . . . . . . . . . . . . 90 
$6.2 \mathrm{Sr}_{3} \mathrm{Ru}_{2} \mathrm{O}_{7}$ : Quantum criticality and its properties . . . . . . . . 92

6.2 .1 Introduction . . . . . . . . . . . . . . . . . 96

6.2.2 Computational Methods . . . . . . . . . . . . . . 102

6.2.3 Results and discussion on the magnetic order . . . . . . . . 103

6.2.4 Summary ......................... 111

7 Electronic structure and transport anisotropy in $\operatorname{Sr}_{3} \mathrm{Ru}_{2} \mathrm{O}_{7}$ in relation to experimental observations . . . . . . . . 113

7.1 Electronic structure of $\mathrm{Sr}_{3} \mathrm{Ru}_{2} \mathrm{O}_{7} \ldots \ldots \ldots \ldots \ldots$

7.2 Electronic transport . . . . . . . . . . . . . . 120

7.3 Summary and conclusions . . . . . . . . . . . . . . 122

8 Summary and concluding remarks . . . . . . . . . . 124 APPENDIX

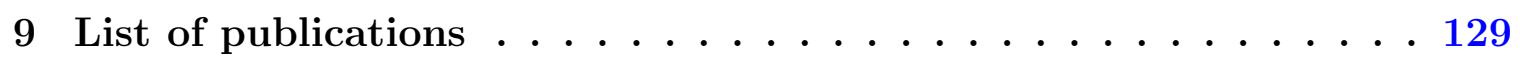

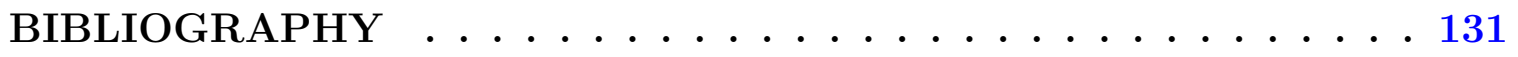

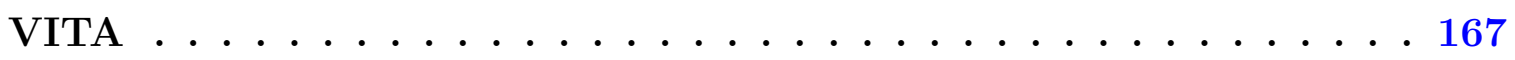




\section{LIST OF TABLES}

Table

3.1 Energy-ordering $\left(\Delta E=E_{\text {anatase }}-E_{\text {rutile }}\right.$ in eV f.u. $\left.{ }^{-1}\right)$ of two $\mathrm{TiO}_{2}$ polymorphs using various exchange-correlation (XC) functionals. . . . 38

3.2 Predicted structure parameters $(\AA)$ of two $\mathrm{TiO}_{2}$ polymorphs using various $\mathrm{XC}$ functionals in relation to experiments. . . . . . . . . . . . .

3.3 Previously known stability order $\left(\Delta E=E_{\text {anatase }}-\mathrm{E}_{\text {rutile }}\right.$ in $\left.\mathrm{eV}\right)$ using various DFT methods. Various types include plane-wave (PW) or linear combination of atomic orbitals (LCAO) basis using pseudopotentials (PP) mostly. For LCAO all-electron method, a triple valence all-electron (TVAE) modification was used with d-symmetry polarization either on $\mathrm{O}$ (denoted by TVAE*) or on both $\mathrm{O}$ and $\mathrm{Ti}$ (denoted by TVAEE $\left.^{* *}\right) .{ }^{\dagger}$ Most accurate basis set reported. . . . . . . . . .

3.4 Calculated Born effective charge tensor for monoclinic $\mathrm{NaSbSe}_{2}$. For $\mathrm{Se}^{*}$, the dual Born charges indicate the relatively opposite alignment of each Se atom. . . . . . . . . . . . . . 
3.5 Dielectric tensor of monoclinic NaSbSe $\mathrm{N}_{2} . \epsilon_{\infty}$ is the electronic whereas $\epsilon_{\mathrm{ph}}$ is the lattice contribution to the total macroscopic dielectric tensor:

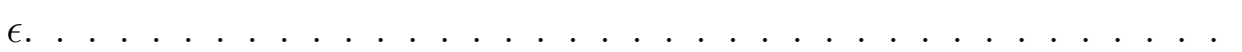

4.1 Used lattice paramaters from experiments with relaxed internal coordinates (in case of $\mathrm{Mg}_{3} \mathrm{Sb}_{2}$ ) . . . . . . . . . . . . .

4.2 Maximum deviation between the estimated and calculated $L(L(|S|)-$ $\left.L\left(k_{\text {open }}\right)\right)$ with relevant doping concentration (conc.) in multiples of

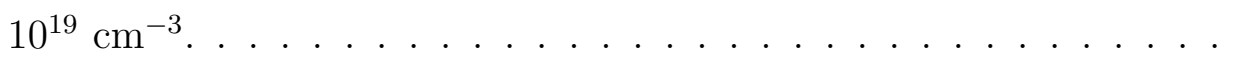

5.1 Structural parameters for Pnma $\mathrm{ZnSeO}_{3}$ and $\mathrm{MnSeO}_{3}$. The lattice parameters, $a, b$ and $c$ are from experiment [139, 140, 141, 142, 143]. Each $M=\mathrm{Zn}$, Mn has six O neighbors, two at each independent bond length. Se has three short bond lengths, with other Se-O distances longer than $2.8 \AA$. There are two independent $M$-O- $M$ bond angles, one connecting octahedra along the $b$ direction (denoted (b)), and two equal angles connecting in the $a c$ plane. . . . . . . . . . . . . .

5.2 Energies of $\mathrm{MnSeO}_{3}$ per formula unit for different magnetic states (refer text). The energy for the lowest energy G-type order was taken as the zero. NSP denotes non-spin-polarized. . . . . . . . . . . . . .

6.1 Relaxed atomic coordinates for $\mathrm{Sr}_{3} \mathrm{Ru}_{2} \mathrm{O}_{7}$. Coordinates are given in the standard setting, Ccce, origin choice 2 for spacegroup number 68 . The lattice parameters from experiment (see text) with this setting are, $a=5.4979 \AA, b=20.7327$ and $\AA, c=5.5008 \AA$. . . . . . . . . . . 104 
6.2 Energy ordering of various magnetic orders (per formula unit) found by LAPW calculations and their (absolute) averaged magnetic moments for both PBE and LDA functionals. Refer to figure 6.6 for naming. FM and nonmagnetic (NM) respectively stand for ferromagnetic and nonmagnetic state (zero-energy level), while all the other orders are antiferromagnetic in nature. Note that the moments reported here are those lying within the LAPW sphere radii. . . . . . . . . . . . 108

6.3 Energetics and magnetic moments for 12 different magnetic orders as shown in figure 6.7 found using both LDA and the PBE functionals. FM and NM respectively stand for ferromagnetic and nonmagnetic orders. . . . . . . . . . . . . . . . . . . . . . . 110

7.1 Density of states (per formula unit) at the Fermi level, $N\left(E_{\mathrm{F}}\right)$ various magnetic orders with both LDA and PBE functionals. Note that for the ferromagnetic order $(\mathrm{FM})$, and for other magnetic orders, $N\left(E_{\mathrm{F}}\right)$ for each single spin channel $(\uparrow, \downarrow)$ is shown. A Gaussian broadening of $4 \mathrm{meV}$ was used. Units are $1 / \mathrm{eV} \ldots \ldots \ldots \ldots \ldots$

7.2 Density of states (per formula unit) value at the Fermi level of $\mathrm{Sr}_{3} \mathrm{Ru}_{2} \mathrm{O}_{7}$ for various magnetic orders under both LDA and PBE functionals obtained using PAW pseudopotential methods. Note that for the ferromagnetic order $(\mathrm{FM}), N\left(E_{F}\right)$ for both spin channels $(\uparrow, \downarrow)$ have been reported. A gaussian broadening 0.0003 Ry was used throughout. . . 117

7.3 In-plane components of the diagonalized reduced electrical conductivity tensor and the corresponding anisotropies for various magnetic orders with the LDA functional. . . . . . . . . . . . . . . 120 
7.4 In-plane components of the diagonalized reduced electrical conductivity tensor and the corresponding anisotropies for various magnetic orders with the PBE functional. . . . . . . . . . . . . . . . . . 121 


\section{LIST OF FIGURES}

Figure

2.1 Schematic diagram showing the components involved in a typical TE

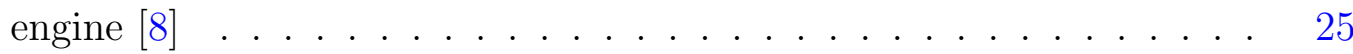

2.2 Plot showing the dependence of various transport quantities on the free carrier concentration $[9] \ldots \ldots \ldots \ldots \ldots \ldots$

2.3 The schematic band structure for the Stoner model of ferromagnetism. The exchange interaction has split the energy of the electrons in either spin channels, enabling it to a selective filling of either bands resulting in an overall ferromagnetism. Source: Wikipedia . . . . . . . . . 30

3.1 Layered structure of ordered $\mathrm{NaSbSe}_{2}$. The yellow spheres are Se, the blue are $\mathrm{Na}$ and the gray ones are $\mathrm{Sb}$ atoms. There are 2 formula units in the unit cell. On left the Se-Sb-Se units are shown. These along with $\mathrm{Na}$ atoms make up the layers. Individual layers are stacked as shown on the right. . . . . . . . . . . . .

3.2 DOS and its projections onto the LAPW spheres shown on a per formula unit basis. . . . . . . . . . . . . . . . . . . . 
3.3 Isoenergy surfaces at $0.05 \mathrm{eV}$ and $0.1 \mathrm{eV}$ below VBM ( $p$-type, left panel) and above conduction band minima [CBM] (n-type, right panel) showing various carrier-pockets. . . . . . . . . . . . . .

3.4 Seebeck coefficients (in $\mu \mathrm{VK}^{-1}$ ) at various temperatures in all three directions as a function of either doping concentration. [Bottom right] reduced conductivity (in $10^{20} \Omega^{-1} m^{-1} s^{-1}$ ) on a log-log scale for both p- (solid lines) and $n$-type (dashed lines) doping. . . . . . . . . .

3.5 [Left Panel] Reduced power factor: $S^{2} \sigma / \tau\left(\right.$ in $10^{10} \mathrm{Wm}^{-1} \mathrm{~K}^{-2} \mathrm{~s}^{-1}$ ) at various temperatures in all three directions as a function of both $p$ - (left half) and $n$-type (right half) doping concentration. Note the difference in scales, especially for y direction of $n$-type. [Right Panel] Calculated EFF (in $10^{-19} \mathrm{~W}^{5 / 3} \mathrm{~ms}^{-1 / 3} \mathrm{~K}^{-2}$ ) in all three directions for monoclinic $\mathrm{NaSbSe}_{2}$ as a function of doping concentration at various temperatures for both $p$ - and $n$-type doping. Note the similar difference in scales.

3.6 Comparison of EFF (in $10^{-19} \mathrm{~W}^{5 / 3} \mathrm{~ms}^{-1 / 3} \mathrm{~K}^{-2}$ ) calculated along direction of best performance for four other known TEs namely $\mathrm{Mg}_{3} \mathrm{Sb}_{2}$, PbTe, GeTe and ZrNiSn with both doping types. Note that at $600 \mathrm{~K}$ we have the rhombohedral structure for the highest EFF value material

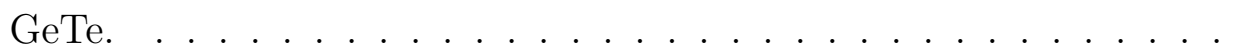

4.1 Calculated $S$ (in $\mu \mathrm{VK}^{-1}$ ) under both CSTA (blue) and APS (red) approximation as a function of doping concentration $\left(\right.$ in $\left.\mathrm{cm}^{-3}\right)$ of either doping types for four TEs at suitable temperatures. The solid lines indicate $p$-type whereas $n$-type doping has been shown in dashed lines. 
4.2 Calculated $L$ (in W $\Omega \mathrm{K}^{-2}$ ) using equation 4.7a under both CSTA and APS approx. similar to figure 4.1 for both doping types. . . . . . .

4.3 First-principles results of $L$ (in $\mathrm{W} \Omega \mathrm{K}^{-2}$ ) vs $p$-doping concentration using equation 4.7b under both CSTA (solid lines) and APS (dashed lines) shown in blue lines for above mentioned TEs at suitable temperatures. The approximation using expression 4.1 have been shown in red similarly. Standard degenerate limit value has been also been shown in black dashed lines. . . . . . . . . . . . . . . . .

4.4 Calculated $L$ (in $\mathrm{W} \Omega \mathrm{K}^{-2}$ ) using equation 4.7b under both CSTA and APS time approximations similar to figure 4.3 for $n$-doped TEs at suitable temperatures. . . . . . . . . . . . . . . . . 68

4.5 The window function: $f^{\prime}\left(E-E_{F}\right)$ plotted vs energy together with its various energy moments all scaled to unity. The doping types have been chosen for the one with better TE performance from literature at a fixed concentration of $5 \times 10^{19} \mathrm{~cm}^{-3}$. The integrand: transport function in equation 4.2 for the CSTA case along with the electronic DOS have also been shown for reference while scaling them appropriately for visibility. A smoothening of 0.003 Ry has been applied for all DOS plots. The zero energy was set at the nearest band edge. . . . . . . . . .

5.1 Structure of $\mathrm{ZnSeO}_{3}$ (left) and $\mathrm{MnSeO}_{3}$ (right). The $\mathrm{Zn} / \mathrm{Mn}$ are in the centers of the octahedra. Note the perovskite structural motif of corner sharing $\mathrm{ZnO}_{6}$ or $\mathrm{MnO}_{6}$ octahedra, although with very strong

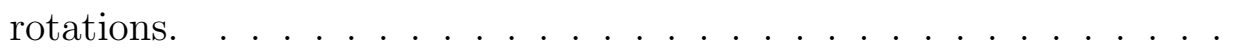


5.2 Electronic density of states and $d$-character projection onto the $\mathrm{Zn}$ LAPW sphere for $\mathrm{ZnSeO}_{3}$, as obtained with the PBE GGA on a per formula unit basis. . . . . . . . . . . . . . . . . .

5.3 Projection of the DOS of $p$ character onto the Se and O LAPW spheres in $\mathrm{ZnSeO}_{3}$ as obtained with the PBE GGA. Note that due to the small Se and O LAPW sphere radii this is approximately proportional to but smaller than the true $p$ orbital characters. . . . . . . . . 80

5.4 Calculated band structure of $\mathrm{ZnSeO}_{3}$ as obtained with the mBJ potential. 80

5.5 Calculated optical absorption spectrum for $\mathrm{ZnSeO}_{3}$ as obtained with

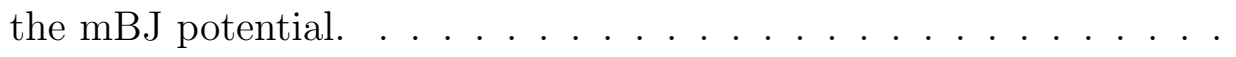

5.6 Calculated electronic density of states and minority and majority spin Mn $d$ projections for $\mathrm{MnSeO}_{3}$ with different magnetic orders. In the ferromagnetic case, majority spin is shown above the axis and minority spin is shown below. . . . . . . . . . . . .

5.7 Calculated band structure of $\mathrm{MnSeO}_{3}$ with G-type antiferromagnetic order, emphasizing majority (left) and minority (right) Mn $d$ character using the 'fat-bands' scheme. . . . . . . . . . . . . . . . . . .

6.1 Schematics of a second order phase transition showing different phases as a function of some tuning parameter $p$ with temperature. The funnel shaped middle part is where the effect of the $T=0 \mathrm{QCP}$ is still felt at higher temperatures. The ordered state on the left has a broken symmetry [Source: Wikipedia] . . . . . . . . . . . . . . . . 
6.2 Reported phase diagram of $\mathrm{Sr}_{3} \mathrm{Ru}_{2} \mathrm{O}_{7}$ in terms of the temperature dependent power-law coefficient $(\alpha)$ of electrical resistance $(\rho)$ as a function of the magnetic field as the tuning parameter at low temperatures. The striking similarity to figure 6.1 is evident. A quadratic temperature dependence confirms its low $T$, low $B$ Fermi-liquid behavior. Note how $\alpha=1$ (non-fermi liquid) regime depresses down towards zero with increasing field. $\alpha$ rises slowly on the other side of high $B$ limit [163]

6.3 Characterizing the sharp change in $\rho\left(=\rho_{\text {res }}+\mathrm{AT}^{\alpha}\right)$ as a divergence of the quantity $A$ when the field sweeps in the vicinity of the metamagnetic field of magnitude $7.85 \pm 0.05 \mathrm{~T}$. Another metamagnetic transition occurs at $\mathrm{B} \sim 5.5 \mathrm{~T}$ for an in-plane $(a b)$ field direction [163]. . . . . . 95

6.4 The schematic phase diagram of $\mathrm{Sr}_{3}\left(\mathrm{Ru}_{1-\mathrm{x}} \mathrm{Mn}_{\mathrm{x}}\right)_{2} \mathrm{O}_{7}$ as a function of Mn doping concentration. Region I is a paramagnetic metallic (PM-M) phase; region II is a paramagnetic insulating (PM-I) phase; region III is a metallic phase with AFM correlation (AFMC-M); and region IV is a long-range AFM insulating phase (LR-AFM-I). The unit cell (with square lattice dimensions) has been shown in inset for reference. [182].

6.5 Crystal structure of orthorhombic $\mathrm{Sr}_{3} \mathrm{Ru}_{2} \mathrm{O}_{7}$, showing layering along $c$ (left) and the view along the $c$ axis, illustrating octahedral rotations (right). . . . . . . . . . . . . . . . 
6.6 Various magnetic configurations investigated. Only Ru atoms are shown. These include ferromagnetism, $A$-type, $G$-type and $C$-type antiferromagnetism, which are common orders for perovskites, two $E$-type orders, which are slightly nondegenerate due to the orthorhombic crystal symmetry, and more complex orders with larger unit cells. . . . . . . 107

6.7 All the magnetic orders investigated in our PAW pseudopotential search strategy. Refer to 6.3 for the detailed energetics for each states. . . . 111

7.1 The temperature dependent difference in the in-plane resistivity $\rho_{\text {aa }}$ and $\rho_{\mathrm{bb}}$ for magnetic fields applied at $\theta=72^{\circ}$ where its in-plane component lies along $a$ direction. More details in ref. [180]. . . . . . . . . 115

7.2 Density of states per formula unit of $\mathrm{Sr}_{3} \mathrm{Ru}_{2} \mathrm{O}_{7}$ for its nonmagnetic state showing the respective LAPW sphere projected contributions from $\mathrm{Ru} 4 d$ and $\mathrm{O} 2 p$ orbitals obtained within LDA. Three kinds of $\mathrm{O}$ contributions (scaled four times for visibility) are observed (see text) with their respective multiplicities, shown in parentheses. The black vertical line at $E=0$ shows the Fermi level. . . . . . . . . . . . . . . . 116

7.3 Projected DOS of $d$ character on a Ru atom in the energy range of the $\mathrm{t}_{2 g}$ bands for the different magnetic orderings as obtained with the PBE functional. Note that the individual $d$ orbitals are mixed because of the low symmetry induced by the octahedral rotations and magnetic order. The same symmetry was used for the E-type and non-spin-polarized. The ferromagnetic, $A$-type, $C$-type, and $G$-type were done in a smaller higher-symmetry cell which leads to a different coordinate system for the $d$ orbitals. . . . . . . . . . . . . . . . . 


\begin{abstract}
Study of the quantum mechanical nature of a material provides invaluable understanding of its underlying mechanisms governing their fascinating novel properties. Density functional based first-principles methods provide us with the necessary tools to approximate the Schrödinger's equation for many-electron periodic solids. Starting from the atomic position of their constituent atoms, using one of the most accurate methods of all-electron calculations, here in this thesis, I present my investigations of several such different materials in light of these novel phenomena.

In the first chapter, I have discussed the basics of this tool and other theoretical concepts that work in the background in order to obtain reliant and consistent results. The results on each of these materials have been arranged around the following six chapters. The second chapter, in its two sections, I demonstrated these methods via two materials: a) the widely known industrial compound $\mathrm{TiO}_{2}$ where I addressed the long standing theory versus experiment disparity of the energy ordering of its two most used polymorphs. Our results, like most of the previous theoretical studies gave anatase as its ground state. In the next section, I investigated the recently synthesized layered monoclinic material: $\mathrm{NaSbSe}_{2}$. The results on its superior electronic and transport property shows its potential as a thermoelectric (TE) candidate. The investigation of TE properties in next chapter focusses on the Lorenz number where a certain widely used prescription for its approximation has been closely examined. Comparing against our first-principles based transport results on few well-known TEs as well as the ideal single parabolic band model, I found that for some materials the prescription works well within acceptable deviations. However, for TEs with complex
\end{abstract}


band structure the deviations are too big which suggests precaution to its use since efficient TEs are often marked by such complex electronic structures only.

The following chapters explore magnetism. Starting with the discussion of the pervoskite compound $\mathrm{MnSeO}_{3}$, we found our results to be predicting its true magnetic ground state order. The study of its energetics and electronic structure, in comparison to its non-magnetic analogue $\mathrm{ZnSeO}_{3}$, its magnetic nature was determined to be of local moment nature. Showing unconventional structural properties for a pervoskite compound, doping and spin-wave dispersion investigations will probably be useful.

In the next two chapters, I focus on the novel material $\mathrm{Sr}_{3} \mathrm{Ru}_{2} \mathrm{O}_{7}$. Widely considered as a classic quantum critical material, I discuss why it is important to understand the nature of fluctuations associated with its quantum critical properties. For this purpose, it is important to know the low-energy metastable states in competition with its ground state. The first-principles investigation based survey yielded the striped $E$-type antiferromagnetic state that lies closest to the ground state. The magnetic-energy ordering in combination to its electronic structure properties, e.g. the density of states suggest its magnetism to be of itinerant nature. My results on the electronic transport indicates that only this striped $E$-type ordered state carries a distinct anisotropy among its in-plane conductivity components. This result is particularly important since the material $\mathrm{Sr}_{3} \mathrm{Ru}_{2} \mathrm{O}_{7}$ is experimentally known to display a similar transport anisotropy of the same order under specific magnetic field. 


\section{Chapter 1}

\section{Introduction}

Understanding the electronic and crystal structure of a material is of paramount importance in materials science. Insights obtained by their observations and thorough study reveal the rich physics driving exotic phenomena in novel materials. This in turn enables us to explain, predict, manipulate and even invent such novel materials. Innovations like transparent solar cells with its potential to replace traditional glass window panes for domestic energy purposes $[1,2]$ or certain cuprates to push the limits of superconductivity transition temperature are to name some of them [3, 4]. Naturally these phenomena and the materials that manifest them hold tremendous potential to address major current challenges. Solving the clean energy crisis, realizing room temperature superconductivity are few such frontier challenges. Phenomenon like thermoelectricity and the role of quantum criticality in unconventional superconductivity are strong candidates for solving these respective challenges. However, they still require major advancements in their experimental realization and theoretical understanding respectively. 
As one would expect, the pursuit of such challenges is met with both theoretical as well as experimental procedures, which go hand in hand. While for experimental methods reliable reproducibility is the key, theoretical procedures however, in addition demand developments from existing concepts with the least possible number of approximations. To investigate such a novel material at the fundamental atomistic level, the entire challenge lies upon solving the Schrödinger's equation for the corresponding multielectronic system from scratch, as accurately as possible. Firstprinciples density functional theory is a powerful theoretical tool to perform this task.

In my research, as presented in this thesis, I explored these two above mentioned phenomena using all-electron density functional calculations tools. The contents of this thesis can be overviewed in the following way. In chapter 2 , the basic details of electronic structure calculations are outlined together with the important theoretical concepts used to tackle their corresponding challenges. Theoretical foundations, namely the Hohenberg-Kohn theorems of the density functional theory are discussed. I will also present few key concepts at the core of my main tool: the linearized agumented plane-wave (LAPW) method. Following this will be a brief exposition to the phenomenon of thermoelectricity. Finally, a short description of magnetic systems is presented along with their localized and itinerant varieties.

In chapter 3 , I will present the results obtained on two different materials where the computational techniques explained in the previous chapter will be demonstrated. In the first section, I will address the long-standing question related to the energy order- 
ing of the two polymorphs of $\mathrm{TiO}_{2}$, a material with widespread industrial use. Using one of the most accurate available methods to settle the long discord between the experimental versus theoretical results, I investigated which form of this compound is the true ground state. The results will be compared to the available literature in relation to the experiments.

In the next section, I will consider the ordered form of the newly synthesized material: $\mathrm{NaSbSe}_{2}$. Through Boltzmann transport theory based on its electronic structure, we will study the material's transport properties. Furthermore, it will be shown how its crystal structure, as well as the chemical composition bestows this material its superior transport properties like high mobility, defect tolerance, etc., the properties essential for thermoelectric and/or solar-cell applications. For this purpose, I will employ a theoretical metric called the electronic fitness function (EFF).

In chapter 4, I will delve further into the thermoelectric phenomenon. Here I will consider the experimental challenge of separating the thermal conductivity into its constituent components in order to obtain the efficiency metric ZT. Making use of the Lorenz number for this purpose, I will closely examine a widely accepted prescription in the literature that is used to address this challenge. Considering some well-known thermoelectrics, I will present the cases for which this prescription to approximate the Lorenz number largely deviates from the value calculated from the microscopic level first-principles.

In the next chapters, I will discuss the phenomenon of magnetism where the localized and itinerant types of magnetism will be explored. Technological developments in 
the field of condensed matter in the last two decades or so has brought up magnetism as an indispensable tool to study the microscopic details of a material's electronic structure. Marked by a rich interplay of charge, spin, lattice and orbital degrees of freedom, certain materials, for example, the ruthenates, the extensive focus of this thesis, often host peculiar properties in their ground states. Thus, understanding these properties starting from a point, especially using first-principles based investigations warrants attention. This is presented in the respective demonstration within two very different perovskite compounds.

First, in chapter 5, I will consider the selenite compounds: $\mathrm{MnSeO}_{3}$ paired with a close comparison to its non-magnetic analogue $\mathrm{ZnSeO}_{3}$, I will find out the key characteristics driving a localized moment system. Based on its crystal properties, it will be shown how this class of material displays unusual properties unlike most other perovskites. Investigations for its characteristic electronic properties leading to magnetism will finally be related to other known materials of interest.

Later, we will observe the class of ruthenates in chapters 6 and 7 . First, we report our investigation of the compound: $\mathrm{Sr}_{3} \mathrm{Ru}_{2} \mathrm{O}_{7}$. Belonging to the class of layered perovskites called the Ruddlesden-Popper (RP) series, all of which display interesting properties, I will first consider this characteristic itinerant system. Experimentally established as a classic material to manifest quantum critical behavior under magnetic field, it hosts various strongly competing degrees of freedom and is observed to show electronic nematicity. Starting with an exposition on quantum criticality, I will use density functional based results that indicate the presence of strong fluctuations 
inherent to such a quantum critical point. Theoretically, I find the states that energetically compete with its ground state and thus strongly influence its ground state properties.

In chapter 7, I discuss the electronic nematicity property. Demonstrated through its computed transport properties, interesting results that resemble its experimentally observed transport anisotropy were found. This leads to the discussion of the implication of these results concerning such experimental observations. 


\section{Chapter 2}

\section{Methods and theoretical background}

The central challenge in first-principles electronic structure for a periodic manyelectron system is to solve its corresponding Schrödinger's equation. For such a system, the Hamiltonian is written as:

$$
H \Psi=\left(-\frac{\hbar^{2}}{2 m} \sum_{i} \nabla_{i}^{2}-\sum_{i, I} \frac{Z_{I} e^{2}}{\left|r_{i}-R_{I}\right|}+\frac{1}{2} \sum_{i \neq j} \frac{e^{2}}{\left|r_{i}-r_{j}\right|}+\frac{1}{2} \sum_{I \neq J} \frac{Z_{I} Z_{J} e^{2}}{\left|R_{I}-R_{J}\right|}\right) \Psi
$$

Here, the lower-case indices are for electronic coordinates whereas the nuclear coordinates are indicated by upper-case indices. Various interactions are represented by these different terms. For an electron of mass $m$, the first term is the total sum of the kinetic energy of all electrons. The second term is the nuclear attraction the individual electrons are subjected due to a positive charge of $\mathrm{Z}$ (atomic number of the element) number of protons in the nucleus. The third term is the similar $1 / r$ 
Coulomb law repulsion between two individual electrons. Finally, the fourth term quantifies the repulsion between two nuclei carrying different charges.

One must note here that equation (2.1) has no contribution of the kinetic energy associated with the movement of the nuclei. This follows from the Born-Oppenheimer approximation which states that given the same amount of kinetic energy, the nuclei, having much larger mass as compared to the electrons, have negligible velocities. As a result, the nuclear coordinates almost stay constant and hence their variation can be neglected. This straightforward approximation helps in reducing the mathematical complexity of the problem within acceptable accuracy. Dropping off the nuclear kinetic energy, it is useful to consider regrouping these terms of the Hamiltonian in a way where the electrons are assumed to be interacting directly to the background positive charge via some $\mathrm{V}_{\text {ext }}$. Introducing concepts like electron shielding, core electrons etc. equation (2.2) can be used to further simplify the problem. Throughout this thesis, we will look more into such approximations.

$$
\hat{H}=-\frac{\hbar^{2}}{2 m} \sum_{i} \nabla_{i}^{2}+\sum_{i} V_{e x t}\left(\mathbf{r}_{i}\right)+\frac{1}{2} \sum_{i \neq j} \frac{e^{2}}{\left|\mathbf{r}_{i}-\mathbf{r}_{j}\right|}
$$

\subsection{Addressing the many body challenge}

In order to obtain the ground state electron configuration of a system defined by the Hamiltonian in equation (2.1), one has to look for a general quantum mechanical solution $\Psi=\Psi\left(\mathbf{r}_{1}, \mathbf{r}_{2}, . ., \mathbf{r}_{i}\right)$ as a function of the electronic coordinates $\mathbf{r}_{i}$ including spins. However, for a typical extended system containing number of electrons in 
the thermodynamic limit, finding such a many-body complex solution $\Psi$ becomes an intractable and cumbersome problem.

Independent electron approximation: The simplest approximation one can make is to assume that the electrons gas of the periodic extended solid is constituted of independent moving electrons occupying some single-electron quantum states: $\psi_{i}$, respecting the periodicity conditions and moving in a Coulomb background of the nuclear positive charge, i.e:

$$
\Psi\left(\mathbf{r}_{1}, \mathbf{r}_{2}, . ., \mathbf{r}_{i}, . ., \mathbf{r}_{N}\right)=\psi_{1}\left(\mathbf{r}_{1}\right) \psi_{2}\left(\mathbf{r}_{2}\right) . . \psi_{i}\left(\mathbf{r}_{i}\right) . . \psi_{N}\left(\mathbf{r}_{N}\right)
$$

This reduces the general many-body equation (2.1) into a one-particle Schrödinger system given by:

$$
-\frac{\hbar^{2}}{2 m} \nabla^{2} \psi_{i}(\mathbf{r})+V(\mathbf{r}) \psi_{i}(\mathbf{r})=\epsilon_{i} \psi_{i}(\mathbf{r})
$$

where the Colulomb interaction involving the electrons has been summed up into the two terms:

$$
V_{\text {nuclear }}(\mathbf{r})=-Z e^{2} \sum_{\mathbf{R}_{I}} \frac{1}{\left|\mathbf{r}-\mathbf{R}_{I}\right|} \& V_{\text {electronic }}(\mathbf{r})=-e \int d \mathbf{r}^{\prime} \rho\left(\mathbf{r}^{\prime}\right) \frac{1}{\left|\mathbf{r}-\mathbf{r}^{\prime}\right|}
$$

In equation (2.5), the left term assumes the electrons to be moving in the background of the array of nuclei at positions $\mathbf{R}_{I}$ while the right term assumes a uniform average potential generated by other (N-1) electrons which leads to the usual electron- 
electron repulsion. Note that $\rho$ is the electronic density at point $r^{\prime}$ and can be obtained as: $\rho(\mathbf{r})=\sum_{i}|\psi(\mathbf{r})|^{2}$. This prescription is known as the Hartree approximation. The electron-electron repulsion which is also the self-interaction energy is approximated as:

$$
E_{\text {Hartree }}=\frac{1}{2} \int d^{3} \mathbf{r} d^{3} \mathbf{r}^{\prime} \frac{\rho(\mathbf{r}) \rho\left(\mathbf{r}^{\prime}\right)}{\left|\mathbf{r}-\mathbf{r}^{\prime}\right|}
$$

However, one finds that the above formulation doesn't respect the Fermi exclusion principle, a fundamental quantum mechanical property of the electrons. It states that two quantum mechanical particles, being the electrons, can't occupy the same quantum state $\psi_{i}$ s.

\subsubsection{Antisymmetric nature of the electron wavefunction:}

The Fermi exclusion principle for a system of $\mathrm{N}$ electrons gives rise to the antisymmetric nature of the many-body wavefunction $\Psi$ that describes its collective state. In other words, swapping the coordinates (could either be the position or the spin coordinate) of two electrons leads to an overall inversion of the wavefunction. Mathematically, $\Psi$ must satisfy:

$$
\Psi\left(\mathbf{r}_{1}, \mathbf{r}_{2}, . ., \mathbf{r}_{i}, . ., \mathbf{r}_{j}, . ., \mathbf{r}_{N}\right)=-\Psi\left(\mathbf{r}_{1}, \mathbf{r}_{2}, . ., \mathbf{r}_{j}, . ., \mathbf{r}_{i}, . ., \mathbf{r}_{N}\right)
$$

This antisymmetrization is effectively a linear superposition of states constructed out of all the product of single electron states [equation (2.3)]. Now, for a system containing $\mathrm{N}$ electrons, any two of them can be swapped in $\mathrm{N}$ ! ways yielding the same number of superposition terms. This procedure of antisymmetrization can be used to 
condense and normalize the many-body wavefunction $\Psi$ into the Slater determinant.

$$
\begin{aligned}
& \Psi_{\text {Slater }}\left(\mathbf{r}_{1}, \mathbf{r}_{2}, . ., \mathbf{r}_{N}\right)=\frac{1}{\sqrt{N !}} \hat{A}\left[\psi_{1}\left(\mathbf{r}_{1}\right) \psi_{2}\left(\mathbf{r}_{2}\right) . . \psi_{N}\left(\mathbf{r}_{N}\right)\right] \\
& \Psi\left(\mathbf{r}_{1}, \mathbf{r}_{2}, . ., \mathbf{r}_{N}\right)=\left|\begin{array}{llll}
\psi_{1}\left(\mathbf{r}_{1}\right) & \psi_{2}\left(\mathbf{r}_{1}\right) & \ldots & \psi_{N}\left(\mathbf{r}_{1}\right) \\
\psi_{1}\left(\mathbf{r}_{2}\right) & \psi_{2}\left(\mathbf{r}_{2}\right) & \ldots & \psi_{N}\left(\mathbf{r}_{2}\right) \\
\ldots \ldots & \ldots & \ldots & \ldots \\
\ldots \ldots & \ldots & \ldots & \ldots \\
\ldots & \ldots & \ldots \\
\psi_{1}\left(\mathbf{r}_{N}\right) & \psi_{2}\left(\mathbf{r}_{N}\right) & \ldots & \psi_{N}\left(\mathbf{r}_{N}\right)
\end{array}\right|
\end{aligned}
$$

Here $\hat{A}$ is the antisymmetrization operator that combines all possible index swaps or equivalently creates the determinant combination of all the product states and is antisymmetric in nature.

\subsection{Density functional theory}

Clearly, it is necessary to formulate a more general but also accurate theory that can be reliably applied to a wide variety of materials, is tractable enough while also being computationally feasible.

As the name suggests, the central theme of the density functional theory is to replace the electronic wavefunctions with its charge density as the fundamental variable. Describing a system of $N$ electrons through wavefunctions requires $3 N$ spatial variables whereas being a scalar quantity, using the charge density only needs three. This possibility of computationally simplifying the many-body problem was enter- 
tained by Hohenberg and Kohn, where by proposing two theorems, they established that in principle, to obtain the ground-state properties of a many-electron system, one may replace wavefunctions with density without any loss of generality [13]. Using the description by equation (2.2), below we discuss these two theorems with their proofs[12].

\subsubsection{The Hohenberg-Kohn theorems:}

Theorem 1: The external potential $V_{\text {ext }}(\boldsymbol{r})$ is uniquely determined (upto an additive constant) as a functional of the ground state electron density $F[\rho(\boldsymbol{r})]$ only.

Corollary: Since the hamiltonian is thus fully determined, except for a constant shift of the energy, it follows that the many-body wavefunctions for all states (ground and excited) are determined. Therefore all properties of the system are completely determined given only the ground state density $\rho_{0}(\mathbf{r})[30]$.

In reference to equation (2.2), for the ground state many-body wavefunction $\Psi$, the unknown functional $F$ is universal for all many-electron densities includes the kinetic energy $(T)$ and the electron-electron Coulomb replusion $\left(V_{e e}\right)$ and is defined as: 


$$
\begin{array}{r}
\hat{H}=F+V_{\text {ext }} \text { with } E=\langle\Psi|H| \Psi\rangle \\
F[\rho(\mathbf{r})]=\left\langle\Psi\left|T+V_{e e}\right| \Psi\right\rangle \text { such that } E[\rho(\mathbf{r})]=\int \rho(\mathbf{r}) V_{e x t}(\mathbf{r}) d^{3} \mathbf{r}+F[\rho(\mathbf{r})]
\end{array}
$$

Proof by reductio ad absurdum:

Let us assume there exists two unique external potential $\mathrm{V}_{\text {ext }}^{1}(\mathbf{r})$ and $V_{\text {ext }}^{2}(\mathbf{r})$ which differ by more than a additive constant which leads to two different ground state wavefunctions: $\Psi_{1}(\mathbf{r})$ and $\Psi_{2}(\mathbf{r})$ and energies $\mathrm{E}_{1}$ and $\mathrm{E}_{2}$ respectively. However, under such assumptions, if they both yield the same ground state charge density $\rho(\mathbf{r})$, then according to the variational principle, we obtain:

$$
\begin{aligned}
E_{1} \leq\left\langle\Psi_{2}\left|H_{1}\right| \Psi_{2}\right\rangle & =\left\langle\Psi_{2}\left|H_{2}\right| \Psi_{2}\right\rangle+\left\langle\Psi_{2}\left|H_{1}-H_{2}\right| \Psi_{2}\right\rangle \\
& =E_{2}+\left\langle\Psi_{2}\left|H_{1}-H_{2}\right| \Psi_{2}\right\rangle \\
& =E_{2}+\int \rho(\mathbf{r})\left[V_{\text {ext }}^{1}(\mathbf{r})-V_{\text {ext }}^{2}(\mathbf{r})\right] d^{3} \mathbf{r}
\end{aligned}
$$

The last step follows from the universality of the functional $F$. Now, if we interchanging indices 1 and 2 in above equation (2.11), and adding them together gives:

$$
E_{1}+E_{2} \leq E_{1}+E_{2}
$$

This emphasizes that the relation holds uniquely only in the case of equality, in which case, the integrand term: $\left[V_{e x t}^{1}(\mathbf{r})-V_{e x t}^{2}(\mathbf{r})\right]$ vanishes. This proves the theorem. 
Theorem 2: The ground state energy, $E[\rho(\boldsymbol{r})]$, a universal functional of the density, may be obtained variationally: the density that minimizes this ground state energy is the exact ground state density, i.e.: $E_{0}=\min _{\rho \rightarrow \rho_{0}} E[\rho(\boldsymbol{r})]$.

Proof[30]: Consider some density $\rho(\mathbf{r})$ for which the total energy functional $E[\rho(\mathbf{r})]$ gives us:

$$
\begin{aligned}
E[\rho] & =T[\rho]+V_{e e}[\rho]+\int V_{e x t}(\mathbf{r})[\rho(\mathbf{r})] d^{3} \mathbf{r} \\
& =F[\rho]+\int V_{e x t}(\mathbf{r})[\rho(\mathbf{r})] d^{3} \mathbf{r}
\end{aligned}
$$

Here $F[\rho]$ is the universal functional as defined above in equation (2.10). Now, for the system corresponding ground state density $\rho^{G S}$, external potential $V_{e x t}^{G S}$ and ground state energy $E^{G S}$, the energy functional is exactly equal to the expectation value of its hamiltonian $\hat{H}$ in the ground state wavefunction $\Psi^{G S}$ :

$$
E\left[\rho^{G S}\right]=E^{G S}=\left\langle\Psi^{G S}|\hat{H}| \Psi^{G S}\right\rangle
$$

Now, for any different density $\rho^{1}$ which corresponds to a different wavefunction $\Psi^{1}$, it follows from the definition of ground state that the corresponding energy $E^{1}$ :

$$
E^{1}=\left\langle\Psi^{1}|\hat{H}| \Psi 1\right\rangle>\left\langle\Psi^{G S}|\hat{H}| \Psi^{G S}\right\rangle=E^{G S}
$$

Thus it follows from equation (2.13) that by minimizing the energy functional in 
the space of density $\rho$ will give us the exact ground state density. Thus theorem 2 is proved.

The strength of these theorems lies in the fact that the problem of solving the many-body Schrödinger's equation is now transformed into an exact problem of variational minimization of the energy functional. However, at the same time, these theorems do not offer any idea as to the nature of this functional $\mathrm{F}$ or how to find it for a given many-electron system. This practical challenge is tackled by the KohnSham formulation which we discuss now.

\subsection{The Kohn-Sham formalism:}

The Kohn-Sham formalism is an essential tool to successfully implement density functional theory in practical systems. It maps the many-body system of $N$-electrons to a fictitious auxiliary system of $N$ non-interacting electrons moving in an effective Kohn-Sham potential $v_{\mathrm{KS}}$ in equation(2.19). The single-particle Kohn-Sham orbitals $\left(\psi_{\mathrm{KS}}\right)$ obtained upon solving such a system is then used to describe all the quantities of interest Note that the Kohn-Sham density is already constrained to yield the same ground state density as the fully-interacting system.

Now, the ground state density $\rho(\mathbf{r})$ being constrained to have the fixed $N$ number of electrons, when used in combination to the variational minimization of the total energy-functional as defined in equation (2.9 and 2.10) gives: 


$$
\delta\left[F[\rho(\mathbf{r})]+\int V_{e x t}(\mathbf{r}) \rho(\mathbf{r}) d^{3} \mathbf{r}+\mu\left(\int \rho(\mathbf{r}) d^{3} \mathbf{r}-N\right)\right]=0
$$

The Lagrange multiplier $\mu$ comes out as:

$$
\mu=\frac{\delta F[\rho(\mathbf{r})]}{\delta \rho(\mathbf{r})}+v_{e x t}(\mathbf{r})
$$

At this point, the universal functional $\mathrm{F}[\rho(\mathbf{r})]$ is broken down into:

$$
F[\rho(\mathbf{r})]=T_{s}[\rho(\mathbf{r})]+E_{\text {Hartree }}[\rho(\mathbf{r})]+E_{x c}[\rho(\mathbf{r})]
$$

Where $E_{\text {Hartree }}$ is the usual classical Hartree term (equ [2.6]) and $T_{s}$ is the kinetic energy of the auxiliary non-interacting electron gas system of density $\rho(\mathbf{r})$ as mentioned above and $E_{x c}$ is called the exchange-correlation energy which is essentially the unknown difference between the kinetic energies of the complex many-electron system and that of the auxiliary single-electron system.

$$
T_{s}=-\frac{1}{2} \sum_{i}^{N} \int \psi_{i}^{*}(\mathbf{r}) \nabla^{2} \psi_{i}(\mathbf{r}) d^{3} \mathbf{r}
$$

Working out the variational principle for the total energy functional explicitly[30], using the chain-rule, we get:

$$
\frac{\delta E}{\delta \psi_{i}^{*}}=\frac{\delta T_{s}}{\delta \psi_{i}^{*}}+\left[\frac{\delta E_{\text {ext }}}{\delta \rho}+\frac{\delta E_{\text {Hartree }}}{\delta \rho}+\frac{\delta E_{x c}}{\delta \rho}\right] \frac{\delta \rho(\mathbf{r})}{\delta \psi^{*}(\mathbf{r})}
$$

$\psi_{i}$ s subjected to the orthonormality constraint: $\left\langle\psi_{i}^{*}(\mathbf{r}) \mid \psi_{j}(\mathbf{r})\right\rangle=\delta_{i j}$

$$
\text { with: } \frac{\delta T_{s}}{\delta \psi_{i}^{*}(\mathbf{r})}=-\frac{1}{2} \nabla^{2} \psi_{i}(\mathbf{r}) ; \frac{\delta \rho(\mathbf{r})}{\delta \psi_{i}^{*}(\mathbf{r})}=\psi_{i}(\mathbf{r})
$$


Till this point, the formalism is exact. The point of separating the total energy in this form is to be able to group together all complex many-particle interactions of the real system into one single term hoping it to be small enough (as compared to the total energy) to be approximated accurately enough.

Using these together, equation (2.14) gives us:

$$
\begin{aligned}
\mu & =\frac{\delta T_{\mathrm{s}}[\rho(\mathbf{r})]}{\delta \rho(\mathbf{r})}+v_{\mathrm{KS}}[\rho(\mathbf{r})] \\
\text { such that: } v_{\mathrm{KS}} & =v_{\mathrm{ext}}+\frac{\mathrm{E}_{\mathrm{Hartree}}}{\delta \rho(\mathbf{r})}+\frac{\delta \mathrm{E}_{\mathrm{xc}}}{\delta \rho(\mathbf{r})} \\
& =v_{\text {ext }}+\mathrm{v}_{\text {Hartree }}+\mathrm{v}_{\mathrm{xc}}
\end{aligned}
$$

The biggest advantage of this simplification is realized when the solution to equation (2.15) is cast in the form:

$$
\left(-\frac{1}{2} \nabla^{2}+v_{\mathrm{KS}}\right) \psi_{i}(\mathbf{r})=\epsilon_{i} \psi_{i}(\mathbf{r})
$$

which essentially are single-particle Schrödinger's equation that can be solved if the form of $v_{x c}$ (or $E_{x c}$ ) is known. $\epsilon_{i} \mathrm{~S}$ are the eigenvalues corresponding to these equations such that the ground state density can always be obtained via:

$$
\rho(\mathbf{r})=\sum_{i}^{N} \psi_{i}^{*}(\mathbf{r}) \psi_{i}(\mathbf{r})
$$




\subsubsection{The exchange-correlation term:}

One must note that the entirety of the Kohn-Sham formalism rests upon the existence of the above mentioned "fictitious" single-particle system for which one can perform all the above simplifications. In other words [30] one asks: is it possible to exactly reproduce the ground state density of a many-electron system using such an auxiliary system? Due to the approximate nature of the functionals, the answer is not known. This however doesn't prevent someone from applying this to real systems. Although the existence of such a system is not guaranteed under the Kohn-Sham formalism, its power lies in the fact that upon applying it to practical systems while using a variety of approximations to the exchange-correlation functional, one obtains results accurate enough that often go beyond the initial purview of such a single-particle approximation.

This leads us to address approximation to the exchange-correlation energy term. As mention earlier, the exchange-correlation term is the collective approximation of all the many-body complex interactions which also includes the inaccuracy in representing the real kinetic energy by the Kohn-Sham kinetic energy $T_{s}$. The way of separating the energy terms as shown in equation (2.16) leaves the exchange-correlation term to be comparatively smaller to the total energy which can then be approximated readily without any major accuracy penalty.

The simplest approximation to achieve this would be to define it in terms of some energy per-electron $\epsilon_{x c}(\mathbf{r})$ that is a local or nearly local functional of the density in the neighborhood of point $\mathbf{r}$ in the following way: 


$$
E_{x c}[\rho(\mathbf{r})]=\int d^{3} \mathbf{r} \rho(\mathbf{r}) \epsilon_{x c}([\rho], \mathbf{r})
$$

In general, the exchange-correlation potential [as defined in equation (2.19)] is the functional derivative of $E_{x c}$ :

$$
V_{x c}(\mathbf{r})=\epsilon([\rho], \mathbf{r})+\rho(\mathbf{r}) \frac{\delta \epsilon_{x c}([\rho(\mathbf{r})], \mathbf{r})}{\delta \rho(\mathbf{r})}
$$

\section{Functionals}

In the Kohn-Sham theory, as stated in equation 2.16, the total energy of a multielectronic system can be expressed as a sum of three terms viz. the total kinetic energy, obtained through the wavefunction derivatives 2.17, the Hartree term for the regular Coulomb repulsion 2.6 and the third and the smallest term, the exchange and correlation term. For a given system, it is the unknown and has to be approximated. It includes the sum total of all the residual electron interaction energies that cannot be expressed directly.

Thus, in order to find the most accurate total energy, various approximations have been proposed. In the following part, we shortly describe a few of such exchangecorrelational functionals that are widely used and have been fundamentally important in the success of density functional theory as a reliable first-principles tool. 
Local density approximation The most fundamental approximation is to introduce a per-electron exchange correlation energy that solely depends on the value of the electronic density of the homogeneous electron gas (HEG) at each point in space, hence the term "local". Motivated from the HEG model, this term is separated individually into the exchange and correlation term. This way, the exchange term can be determined analytically in terms of this density.

The remaining correlation term is analytically determined from the high or low density limit of the electron gas in terms of the Wigner-Seitz radius $r_{s}$, given by: $\frac{4}{3} \pi r_{s}^{3}=\frac{1}{\rho}$. Combined with accurate quantum Monte-Carlo data [14], the correlation energy is determined for any intermediate density values. Using these approximations, many functionals have been proposed e.g. by Vosko-Wilk-Nusair (1980) [22], Perdew-Zunger (1981) [21], Perdew and Wang [15]. Interestingly, LDA works remarkably well for materials where the electron density is slowly varying. In a wide variety of materials, LDA works especially well in the calculations of equations of state, elastic constants, vibrational energies etc. But for systems with strong electron correlation, for example, transition metal oxide like $\mathrm{NiO}$ or Mott insulators, it yields erratic results. A common trend is the tendency to overbind systems which leads it to predict shorter bond lengths etc.

Generalized gradient approximation In order to incorporate the strongly varying electronic density in practical materials, a direct way to improve the accuracy of the exchange correlation functionals is to include its gradient information along with its local value $\left(n_{\mathbf{r}}\right)$ at a point in the space. This is being taken care of by the 
class of generalized gradient approximation or GGAs:

$$
E_{x c}^{G G A}[n(\mathbf{r})]=\int n(\mathbf{r}) \epsilon_{x c}^{h o m}[n(\mathbf{r})] F_{x c}[n(\mathbf{r}), \nabla n(\mathbf{r})] d \mathbf{r}
$$

The $F_{x c}[n(\mathbf{r}), \nabla n(\mathbf{r})]$ is known as the enhancement factor. As opposed LDA, being derived from the HEG model, there is no direct analytic expression for GGAs, and depending on their enhancement factor, each of these have their own forms. However, their formulation includes certain constraints that they must follow. It is important to note that the pair density $P\left(\mathbf{r}, \mathbf{r}^{\prime}\right)$ gives the probability of simultaneously finding an electron at the point $\mathbf{r}$ within volume element $d \mathbf{r}$, and another electron at $\mathbf{r}^{\prime}$ in volume element $d \mathbf{r}^{\prime}$, among the other $N-2$ electrons in the system. The total $N$ number of electrons for the system constraint gives: $\iint P\left(\mathbf{r}, \mathbf{r}^{\prime}\right) d \mathbf{r}^{\prime} d \mathbf{r}=N(N-1)$ Due to Fermi exclusion principle, the effect of exchange-correlation interaction of an electron is to create a depletion, or hole, of electron density around itself. Considering this hole, the pair-density function is written as: $P\left(\mathbf{r}, \mathbf{r}^{\prime}\right)=n(\mathbf{r}) n\left(\mathbf{r}^{\prime}\right)+n(\mathbf{r}) n_{\mathrm{XC}}\left(\mathbf{r}, \mathbf{r}^{\prime}\right)$ where $n_{\mathrm{XC}}\left(\mathbf{r}, \mathbf{r}^{\prime}\right)$ is the exchange-correlation hole density. Due to this, the $n_{\mathrm{XC}}$ follows the important normalization criteria known as the sum rule: $\int n_{\mathrm{XC}}\left(\mathbf{r}, \mathbf{r}^{\prime}\right) \mathrm{d} \mathbf{r}^{\prime}=-1$. This condition can be physically interpreted as the "hole" carrying the total deficit of one electron around itself [16]. Separating the exchange and correlation into their individual contributions, $n_{\mathrm{XC}}\left(\mathbf{r}, \mathbf{r}^{\prime}\right)=\mathrm{n}_{\mathrm{X}}\left(\mathbf{r}, \mathbf{r}^{\prime}\right)+\mathrm{n}_{\mathrm{C}}\left(\mathbf{r}, \mathbf{r}^{\prime}\right)$ leads to two separate sum rules:

$$
\int n_{\mathrm{X}}\left(\mathbf{r}, \mathbf{r}^{\prime}\right) d \mathbf{r}^{\prime}=-1 ; \int n_{\mathrm{C}}\left(\mathbf{r}, \mathbf{r}^{\prime}\right) d \mathbf{r}^{\prime}=0
$$

These important constraints are respected in a GGA like the PBE.The GGA fun- 
cational proposed by Perdew-Burke-Ernzerhof (PBE) [17] is among the most widely used GGAs and has been put to use in this thesis many times. The simplistic form of its enhancement factor and the fact that all parameters (other than those in local spin density) are fundamental constants, combined with its accuracy for the case of a variety of atoms, molecules and solids make it a favorite choice. Other well-known GGAs include: Perdew-Wang (1991) [26], Wu-Cohen (2006) [19], PBEsol (2008) [20] etc. As compared to LDA, the GGAs work better for magnetic systems.

\subsubsection{The choice of basis}

The above mentioned Kohn-Sham formalism, combined with the periodicity of the lattice greatly simplifies the many-electron problem. However, in order to fully describe and perform these calculations, one needs to expand the Kohn-Sham wavefunctions which in turn describes the density. For this purpose, one requires a certain basis set of expansion functions.

Again, stemming from the independent electron approximation, the most natural choice is to use a finite set of orthogonalized planewaves by choosing an appropriate energy cutoff. Although easier to formulate, the highly localized nature of the core electrons with their rapidly varying orbital characters demand a very high cutoff for these planewaves, which in turn increases total computation times. Fortunately, these core electrons are not chemically active and do not really affect the materials' overall electronic characteristics. This leads one to approximate these core wavefunctions in a computationally cheaper way.

The widely used pseudopotential approach removes the core electron density and 
replaces it with an approximate density following some physical and mathematical constraints. This forms the basis of the so called frozen core approximation, the method which is based on the transferability of the pseudopotential [27]. Chapter 3 , section 3.1 is aimed at resolving the long-standing challenge of theory versus experimental mismatch of the energy ordering of $\mathrm{TiO}_{2}$ polymorphs by questioning this frozen core approximation.

However, a sound theoretical approach aims to remove ad-hoc approximations which lead one to use "all-electron" methods. The linearized augmented planewave (LAPW) methods [6], used throughout this thesis to obtain the electronic ground state of a material, is one such tool. The LAPW technique is fundamentally a modification to the original augmented planewave (APW) method of Slater [28]. The APW method utilizes the same fact that near an atomic nucleus the potential and wavefunctions are strongly varying but nearly spherical. However, the interstitial space between these atoms, both of them are smoother and hence can be expanded in terms of planewaves accurately. Therefore, the space is divided into regions of atom centered "muffin-tin" spheres $(S)$ inside which radial solutions to the Schrödinger equation $\left(u_{l}\right)$ with spherical harmonics are used as the basis whereas regular planewaves are used in the remaining interstitial region $(I)$ as shown below ${ }^{1}$ :

$$
\psi_{\mathbf{k}}(\mathbf{r})=\left\{\begin{array}{cc}
\Omega^{-1 / 2} \sum_{\mathbf{G}} c_{\mathbf{G}} e^{i(\mathbf{G}+\mathbf{k}) \cdot \mathbf{r}} & r \epsilon I \\
\sum_{l m} A_{l m} u_{l}(r) Y_{l m}(\hat{r}) & \text { r } \epsilon S
\end{array}\right.
$$

\footnotetext{
${ }^{1}$ Gs are the reciprocal lattice vectors
} 
However, for the kinetic energy to remain well-defined, the functions in these two regions must be continuous at the sphere boundary. Expanding the planewave in terms of the spherical harmonics and truncating the expression up to some finite $l$ value gives us the expansion coefficients $\left(A_{l m} \mathrm{~s}\right)$ for the region inside the spheres. However, the energy factor $E_{l}$ in the wavefunction form leads to complications where they have to be fixed at band energies such that the resultant basis functions are energy dependent and computationally expensive in general [6, 29].

To overcome this, the basis functions inside the spheres are defined as the linear combination of the radial functions, $u_{l}(r) Y_{l m}(\hat{r})$ and their derivatives with respect to the linearization parameters $E_{l}$ as:

$$
\psi_{\mathbf{k}}(\mathbf{r})=\left\{\begin{array}{cc}
\Omega^{-1 / 2} \sum_{\mathbf{G}} c_{\mathbf{G}} e^{i(\mathbf{G}+\mathbf{k}) \cdot \mathbf{r}} & r \epsilon I \\
\sum_{l m}\left[A_{l m} u_{l}(r)+B_{l m} \dot{u}_{l}(r)\right] Y_{l m}(\hat{r}) & \mathrm{r} \epsilon S
\end{array}\right.
$$

In this case, $\mathrm{A}_{l m} \mathrm{~s}$ and $\mathrm{B}_{l m} \mathrm{~s}$ are obtained by matching both the value and slope of the basis function at the sphere surface. In comparison to the APW method, this generalization removes the restriction of fixing $E_{l} \mathrm{~s}$ at the cost of introducing smaller errors in solving the secular equation [6, 29]. The method used herein the thesis relies upon the technique of introducing the so-called "local-orbitals" leading to the LAPW+lo approach which lifts all restrictions and can then be used for any arbitrary material as one of the most accurate, generally applicable techniques available for electronic structure calculations. 


\subsection{Brief exposition of the explored phenomena}

In this section, I briefly introduce the basic concepts concerning various phenomena that are explored in the rest of this thesis. These can be broadly categorized into: thermoelectricity and magnetism in the context of quantum criticality. Being closely related to the material's microscopic electronic structure, these properties, as will be seen in further sections, are crucial characteristics which upon investigation leads us to explain and predict many of their macroscopic properties.

\subsubsection{Thermoelectricity}

In order to address the growing need of clean energy, finding its alternate sources is one of the burning challenges of current times. Thermoelectricity, as the name suggests is the property of a material that enables it to convert thermal energy into useful electrical work. Given its potential, thermoelectricity can be used to harvest waste heat, thus improving the overall efficiency of any conventional heat based engine. Not to mention its scalability as well as the absence of any moving parts already make it a useful choice in aerospace and military applications.

Following figure 2.1 shows the basic parts involved in the design of a typical engine. The legs of the engine, labeled as $p$ - and $n$-type are the TE materials which when placed across the upward pointing temperature gradient, generates an electric voltage gradient $\mathrm{E}$ in the direction as shown. Connecting it to an external load gives us an electric current that can perform useful electrical work on an external load. 


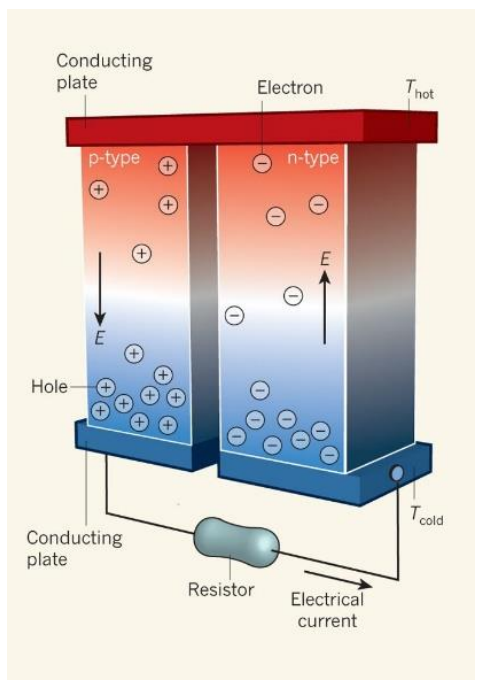

Figure 2.1: Schematic diagram showing the components involved in a typical TE engine [8]

A major goal in TE research involves finding suitable materials to construct these legs. In order to quantify the degree of performance for a candidate material, for a simplified TE device, one requires the dimensionless figure-of-merit, $Z T$, given by:

$$
Z T=\frac{S^{2} \sigma}{\kappa} T
$$

Here $S$ stands for the Seebeck coefficient, $\sigma$ is the electrical conductivity together giving us the power factor $S^{2} \sigma$ and $\kappa=\kappa_{e}+\kappa_{l}$ is the thermal conductivity comprised of the electronic thermal conductivity $\left(\kappa_{e}\right)$ and the lattice or the phononic thermal conductivity $\left(\kappa_{l}\right)$. We will explore more about these contributions to the thermal conductivity later.

For a working thermoelectric, its efficiency $(\eta)$ is defined as: 


$$
\eta=\frac{\text { Electrical energy dispersed at the load resistor }}{\text { Thermal energy intake at the hot junction }}
$$

The $Z T$ factor, as defined above, is required to find the theoretical maximum efficiency of a TE device. It is given by:

$$
\eta_{\max }=\frac{\mathrm{T}_{\mathrm{H}}-\mathrm{T}_{\mathrm{C}}}{\mathrm{T}_{\mathrm{H}}} \frac{\sqrt{1+Z \mathrm{~T}_{\mathrm{avg}}}-1}{\sqrt{1+Z \mathrm{~T}_{\mathrm{avg}}}+\frac{\mathrm{T}_{\mathrm{C}}}{\mathrm{T}_{\mathrm{H}}}}
$$

Where $\mathrm{T}_{\mathrm{H}}, \mathrm{T}_{\mathrm{C}}$ are the temperature at the hot end and cold end and $\mathrm{T}_{\mathrm{avg}}$ is the average of these two. The preceding term is the Carnot efficiency [10]. State of the art TE materials like $\mathrm{Bi}_{2} \mathrm{Se}_{3}, \mathrm{Sb}_{2} \mathrm{Te}_{3}$ and their alloys have a typical peak $Z T$ value in the range of 0.8 to $1.1[11]$.

The Seebeck coefficient is the fundamental measure of the thermoelectric effect defined as the voltage generated per degree of temperature gradient. Broadly, insulators tend to have a high S. However, a well-performing thermoelectric also requires a high electrical conductivity in order to facilitate a larger flow of current. Thus, a high power factor is always desirable. Nevertheless, such a thermoelectric, without its low thermal conductivity will tend to leak heat across the heat source and sink, bringing down its overall efficiency. So, altogether, $Z T$ is what quantitatively determines the degree of performance of a TE.

In the following figure 2.2 we show how various transport properties vary upon the availability of free charge carriers. On the left, we have insulators with high S whereas metals tend to have a very high number of free electrons yielding them a high 
$\sigma$. These electrons also facilitate a larger heat transfer. So, being able to maximize the power factor combined with a low $\kappa$ presents one of the central challenges in $\mathrm{TE}$ research. Making note of all three of these dependencies, one finds semiconductors to have an ideal number of carrier concentration leading to a high $Z T$ value. In practice, tuning a pristine semiconductor TE material's carrier concentration via doping is one of the techniques used to achieve high $Z T$ (as seen in the $p$ - and $n$-type labeled legs in figure 2.1).

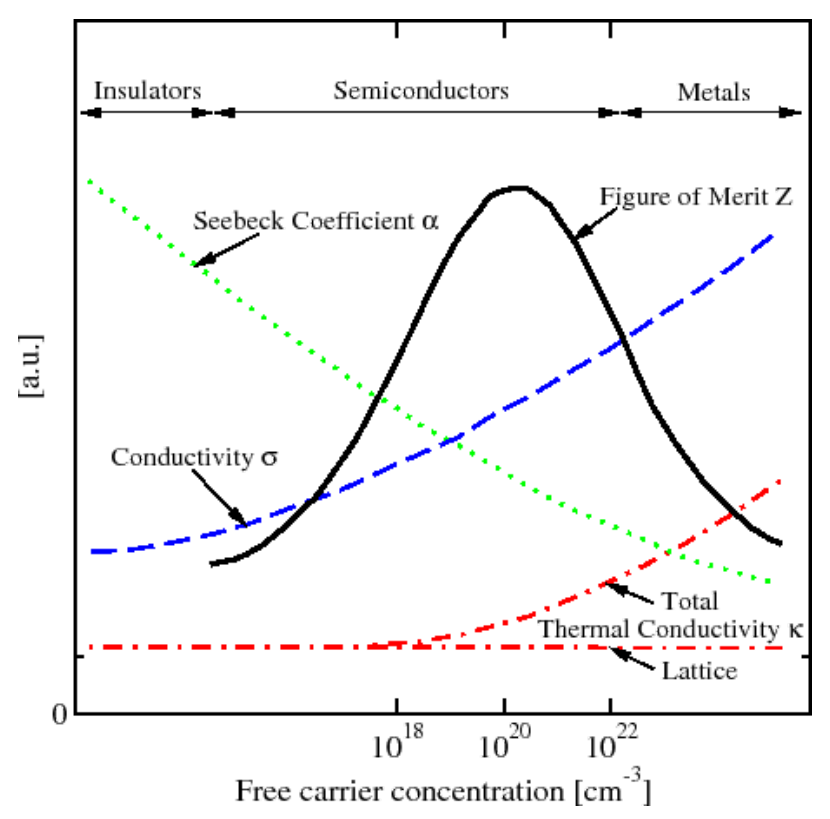

Figure 2.2: Plot showing the dependence of various transport quantities on the free carrier concentration [9].

In the later chapters of this thesis, I will explore a few of these semiconductors TEs and their transport properties in detail. Through first-principles calculations, the inherent properties of their electronic structure that make them good TE materials will be found out. 


\subsubsection{Magnetism}

The source of magnetism in a condensed matter systems is ultimately the magnetic moments of their individual electrons, arising from their spin and angular orbital momenta. It is the overall arrangement of these electrons that finally decide the macroscopic nature of magnetism in a material. As a result, studying magnetism reveals immense information about various properties of a material. I will discuss here two of the main and opposing types of magnetism: localized and itinerant magnetism. As its name suggests, the localized magnetism finds its origin from distinct electron spins, from various orbitals located at fixed points while interacting with one another. However, the itinerant magnetism, loosely translating to 'moving' magnetism, arises as a result of the collective behavior of the entire electron gas, localized in the momentum space. Hence, the underlying interactions among these electrons are closely related to the nature of the Fermi surface itself. In the following paragraphs, we discuss in short, the mechanism working behind each of them.

\section{Local moment magnetism}

Weiss introduced the notion of an interaction between atomic magnetic moments in solids and approximated its effect by a mean molecular field proportional to the average magnetization [205]. This interaction resulting to the alignment of these moments leads to macroscopic magnetism. Heisenberg mentioned this mean-field to a quantum mechanical exchange interaction $J_{i j}$ which we also know as the Heisenberg 
model. For lattice sites $i$ and with atomic spin operator $\hat{S}_{i}$, the model defined the Hamiltonian in terms of this interatomic exchange interaction given by:

$$
H=\sum_{i j} J_{i j} \hat{\mathbf{S}}_{i} \hat{\mathbf{S}}_{j}
$$

The Curie-Weiss law: $\chi \propto \frac{1}{T-T_{C}}$, governing the magnetic susceptibility $(\chi)$ of a ferromagnet above its Curie temperature $\left(T_{C}\right)$, is naturally explained within this model. It is worth noting that for localized magnetic systems, the saturation magnetic moment, if an integer multiple of the Bohr magneton $\left(\mu_{\mathrm{B}}\right)$. Chapter 5 discusses the details of one such localized magnetic system where superexchange interaction is found to be prevalent.

\section{Itinerant moment magnetism}

The magnetism in many elemental $3 d$ metals, for example, $\mathrm{Fe}$, Co, $\mathrm{Ni}$, etc., is explained by itinerant magnetism. Due to its origin which is not derived from electron(s) on the lattice sites, their saturation magnetic moment is often not an integer multiple of $\mu_{\mathrm{B}}$, accompanied with usually higher saturation fields. One way to explain this is through the Stoner mechanism, effective in systems that have a signature high value of density of states at the Fermi level leading to Stoner ferromagnetism. Arising from the electron-electron repulsion, the Stoner model can be formulated in terms of an energy split from the dispersion relation between the electrons of the opposite spin channels as: 


$$
E_{\uparrow}(k)=\epsilon(k)-I \frac{N_{\uparrow}-N_{\downarrow}}{N}, \quad E_{\downarrow}(k)=\epsilon(k)+I \frac{N_{\uparrow}-N_{\downarrow}}{N}
$$

where the difference term is based on the exchange energy governed by the Stoner parameter $I, N_{\uparrow \text { or } \downarrow} / N$ is the dimensionless quantity of spin up/down electrons and $\epsilon(k)$ is the dispersion relation of the spinless system. For a fixed number of spin up/down electrons, the corresponding energies of the electrons in either spin channels can be calculated in terms of the polarization, $P=\left(\frac{N_{\uparrow}-N_{\downarrow}}{N}\right)$. Systems that have a density of states at Fermi level higher than the inverse of this exchange energy parameter $I$, the Stoner criterion, i.e.: $N\left(E_{F}\right)>\frac{1}{I}$, spontaneously form a polarized state giving rise to an overall ferromagnetic arrangement as shown in figure 2.3.

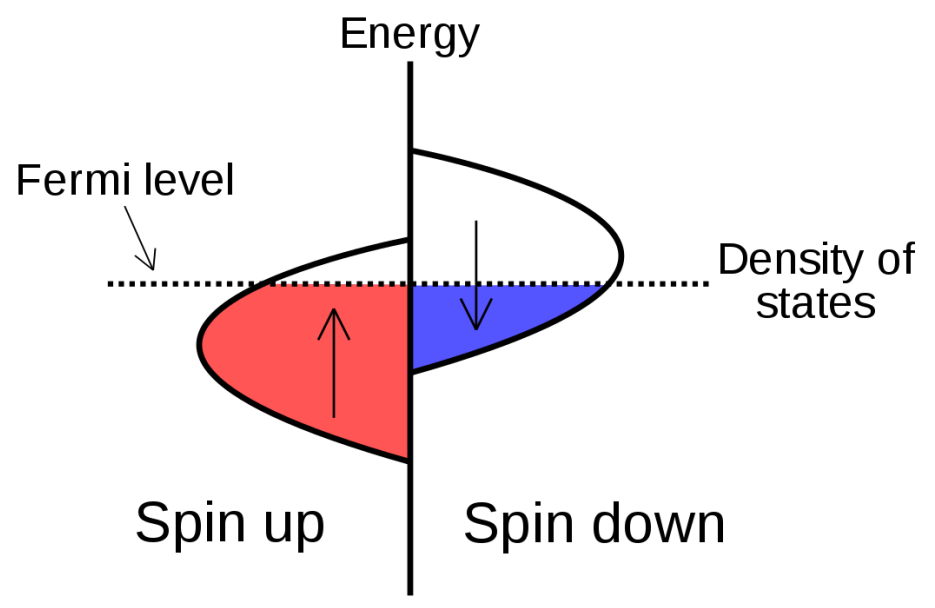

Figure 2.3: The schematic band structure for the Stoner model of ferromagnetism. The exchange interaction has split the energy of the electrons in either spin channels, enabling it to a selective filling of either bands resulting in an overall ferromagnetism. Source: Wikipedia

It must be noted that itinerant magnetism is a many-body phenomenon and these systems can often host exotic properties. For example, Cr is the classic itinerant an- 
tiferromagnet with an incommensurate spin density wave. Here the spin fluctuations, localized in the momentum space play the pivotal role.

In terms of first-principles results, the difference between the localized and itinerant moment magnetism is often clearly shown by the energetics between the individual magnetic orders. In a localized magnetic system, the moments are usually large and due to a high energy of formation of these moments, the paramagnetic state energetically lie much higher than the magnetically ordered states. However, in a localized magnetic system, such a distinction is not always present. The energy ordering of different states is often seemingly haphazard to the point that certain magnetic orders might not even be present as a self-consistent solution to the Kohn-Shan equations. Details of one such magnetic system, namely $\mathrm{Sr}_{3} \mathrm{Ru}_{2} \mathrm{O}_{7}$ has been discussed in chapters 6 and 7. 


\section{Chapter 3}

\section{Demonstration of first-principles tools: Case studies of two different materials}

In this chapter, we make use of the theoretical methods covered in the previous chapter. The work here illustrates some of the important challenges that also demonstrates the strengths of first-principles calculations. In particular, in the first part, identifying the ground state of $\mathrm{TiO}_{2}$ is a challenge that remains unsolved due to the approximate nature of the theory and limitations of functionals, while the ability of these approximate methods to treat the physical and chemical complexity of interesting materials allows one to extract insights into the origins and chemical basis of technologically interesting materials, such as the ternary semiconductors discussed in the later part of this chapter.

Initially, we discuss the widely known industrial material $\mathrm{TiO}_{2}$. It is naturally found mainly in its three polymorphs. Here, we address the long-standing ques- 
tion of energy ordering of two of them, viz. rutile and anatase. With our accurate first-principles tools, we tried to resolve the conflicted findings across theory and experiments so to determine which of these two polymorphs is the true ground state of the material.

We compared the results of both theoretical and experimental investigations of this well-known material from the literature and compared them with our precise, well-converged all-electron calculations. Through this work, we demonstrated the role of various calculation parameters so as to obtain accurate energetics between these two forms of the material.

In the later part, we explore another material, the well-ordered, layered compound: $\mathrm{NaSbSe}_{2}$. Previously known in the literature in its cubic amorphous form, nanocrystals of this material were recently synthesized in its ordered form intended for photovoltaic applications. From the experimentally estimated band gap combined with our ab-initio electronic structure results, we find that the material possesses many properties that results in good electronic transport. In combination of all these findings, we conclude that layered $\mathrm{NaSbSe}_{2}$ can potentially be a thermoelectric material. 


\subsection{Ground state energy ordering of $\mathrm{TiO}_{2}$ poly- morphs}

\subsubsection{Introduction}

Titanium dioxide, a widely used industrial chemical is known to exist in several structural forms known as its polymorphs. Mostly known three of them are: rutile, anatase and brookite. While rutile is mostly used to manufacture pigments and is found to have properties useful as a thermoelectric, anatase, being a semiconductor finds its diverse use in photocatalysis, photovoltaics etc.

Scientifically this material has been a subject of investigation for over last four decades including many studies to determine the true ground state polymorph of the material. Calorimetric studies by Ranade and co-workers [31] found the energy difference $\Delta E=0.027 \mathrm{eV}$ where $\Delta E=E_{\text {anatase }}-E_{\text {rutile }}$ per formula unit $\mathrm{TiO}_{2}$ at 975 K. In other works using high temperature oxide melt solution calorimetric methods at atmospheric pressure, $\Delta E$ was found to be $0.034 \mathrm{eV}$ at $971 \mathrm{~K}[32]$ and $0.068 \mathrm{eV}$ at $968 \mathrm{~K}$ [33]. Mitsuhashi and Kleppa in their same study [32] reported $0.030 \mathrm{eV}$ at $1360 \mathrm{~K}$ using differential scanning calorimetry whereas Levchenko and co-workers found $\Delta E=0.018 \mathrm{eV}$ [34] at $1360 \mathrm{~K}$ using drop solution calorimetry. Clearly, diverse experimental methods concurred on the fact that rutile was the ground state of $\mathrm{TiO}_{2}$ at high temperatures. Although effect of several experimental conditions like grain size [35], phase control [36], water-content [34], reaction atmosphere [37], presence of agents like $\mathrm{Al}_{2} \mathrm{O}_{3}[38]$ and $\mathrm{SiO}_{2}[39,40]$ etc. have been studied, but all of them found rutile to be more stable than anatase at indicated high temperatures within the above 
described scatter of experimental values. Finally, Smith and co-workers [41] reported that over entire lower temperatures ranging from 0 to $1300 \mathrm{~K}$, bulk anatase does not have a thermodynamic stability field over rutile.

On the flip side however, theoretical studies indicates otherwise where various investigations using pseudopotential based density functional methods using both local density and generalized gradient approximations found anatase to be more stable over rutile. For instance, using a plane-wave basis sets, Mikami and co-workers [42] found $\Delta \mathrm{E}=-0.060 \mathrm{eV}$ or $+0.049 \mathrm{eV}$ within the LDA, depending on the type of their pseudopotential used whereas Milman [43] reported -0.06 eV within GGA. In other works, $\Delta \mathrm{E}$ values of -0.032 to $-0.036 \mathrm{eV}$ and +0.016 to $+0.098 \mathrm{eV}$ were reported using Gaussian linear combination of atomic orbitals (LCAO) basis sets, depending on the details of the basis and functionals employed. Even using only exchange based Hartree-Fock calculations within both LCAO [44] and pseudopotential studies [45] found similar results. Using random phase approximation however, Cui and co-workers [46] found anatase to be more stable over rutile. Finally, beyond density functional methods, Luo and co-workers [47] found $\Delta E=-0.059 \mathrm{eV}$ using diffusion quantum Monte Carlo (DQMC) calculations with the inner core described by a pseudopotential. In addition, using DQMC again, Trail and co-workers [48] reported similar results where anatase was found $\Delta E=-0.018 \mathrm{eV}$ to be slightly less albeit more stable over rutile. One must note that DQMC is generally regarded as the most accurate method for solving the Schrödinger's equation and importantly does not suffer from the biases that may be inherent in ad-hoc corrections to approximate DFT. 
To summarize the literature on the stability of $\mathrm{TiO}_{2}$, the bulk of these results points to either an issue with the experimental ground state determination or some fundamental issue in commonly used approximate density functional methods and/or some overlooked approximation. An important factor to note here is the use of different approximations methods used for the core-states in the DQMC studies [47, 48]. Considering the accuracy of DQMC methods, one such possibility is with the use of approximations in the treatment of the outer core states, e.g. with pseudopotentials and the frozen core approximation. In $\mathrm{TiO}_{2}$, correct treatment of these outer core states, especially the $3 p$ state is essential for predictions of some properties such as the electric field gradients (EFGs) [49].

It is also interesting to note that many theoretical studies $[50,51,52]$ predicted the lattice parameters of both of these polymorphs to be in close agreement with the experimental data, yet on the contrary, the energy ordering was dependent on the methods used [44, 42] and mostly contradicted the reported experimental ground state.

In this work, we address this question of rutile-anatase energy ordering. We used the accurate available all-electron full potential linearized augmented planewave (LAPW) plus local orbital (LO) method [6] within density functional theory and focus on the bulk energy ordering. This allows us to mitigate issues related to basis sets and pseudopotentials, since the method has no pseudopotentials and the basis sets and potentials are expanded in highly precise general forms that can be tested 
and systematically improved, e.g. by changing sphere radii, increasing the cutoffs and adding local orbitals.

\subsubsection{Computational details}

This investigation reports full-potential LAPW+lo calculations performed using the WIEN2k code [5]. The atomic sphere radii for Ti and $\mathrm{O}$ were chosen at $1.95 \mathrm{Bohr}$ and 1.55 Bohr respectively after being checked against their individual variations. The planewave sector basis set size was cutoff set by choosing $R_{\min } \mathrm{K}_{\max }=7.5$ where $R_{\min }$ stands for the atomic radii for the smallest atom, being $\mathrm{O}$ in this system. Given this choice of the sphere radii leads to effective $R_{\mathrm{Ti}} \mathrm{K}_{\max } \approx 9.5$ for treating metallic $\mathrm{Ti}$ and was confirmed after performing convergence tests with $\Delta E$ variations below $\sim$ $0.4 \mathrm{meV}$. Such a choice of $R K_{\max }$ has been made throughout this thesis to accurately treat metal atoms. As mentioned earlier, we chose LAPW+lo basis set over APW+lo since it is more flexible at the cost of a slightly larger size of the plane-wave basis set.

For performing Brillouin zone (BZ) integration, $k$-point grids of size $10 \times 10 \times 10$ for anatase and $10 \times 10 \times 16$ for rutile were used. This size of BZ sampling was also carefully tested against a convergence threshold lower than $0.04 \mathrm{meV}$. Thus all different calculation parameters were fixed. Structures were optimized in two separate methods, i.e:

1) fixed the lattice parameters to experimental values while relaxing the internal atomic positions and

2) fully relaxing both the lattice parameters and atomic coordinates to perform a global minimization. 
The calculations here were performed using four different functionals. In particular, the LDA [15] and three different GGAs viz.: Perdew-Burke-Ernzerhof [17] (PBE) GGA, the Wu-Cohen [19] (WC) GGA and the PBEsol [20] GGA.

\subsubsection{Results and discussion}

Our calculations to obtain the energy-ordering of the material always yielded anatase to be the more stable over rutile, irrespective of the exchange-correlation functional used or the way lattice parameters were optimized. Interestingly, the predicted lattice parameters were generally in close agreement with the experiment $[53,54]$. The obtained energy values have been reported below in Table 3.1.

Table 3.1: Energy-ordering $\left(\Delta E=E_{\text {anatase }}-E_{\text {rutile }}\right.$ in eV f.u. $\left.{ }^{-1}\right)$ of two $\mathrm{TiO}_{2}$ polymorphs using various exchange-correlation (XC) functionals.

\begin{tabular}{lll}
\hline \hline XC func. & $E_{\exp }$ & $E_{\text {relax }}$ \\
\hline LDA & -0.032 & -0.025 \\
PBE & -0.096 & -0.101 \\
Wu-Cohen & -0.058 & -0.057 \\
PBEsol $[20]$ & -0.061 & -0.061 \\
\hline \hline
\end{tabular}

One may note that in Table 3.1 the PBE GGA gives an energy difference 2-4 times larger than other functionals which is similar to previous calculations. The PBE functional was designed to improve energies using exact constraints and is almost invariably superior to the LDA for energies $[17,18]$. For example, the high pressure transitions of $\mathrm{SiO}_{2}$ polymorphs are much improved by the GGA [55]. Thus it is interesting to find a large stabilization of anatase over rutile with this GGA. 
The calculated lattice parameters have been reported in Table 3.2 along with the internal coordinates as $z$ coordinate of oxygen in anatase and $x$ and $y(=u)$ for rutile. It may be noted that these energetics do not show much difference due to the structure relaxation.

Table 3.2: Predicted structure parameters $(\AA)$ of two $\mathrm{TiO}_{2}$ polymorphs using various $\mathrm{XC}$ functionals in relation to experiments.

\begin{tabular}{ccccccc}
\hline \hline \multirow{2}{*}{ XC func./expmt. } & \multicolumn{3}{c}{ Anatase } & \multicolumn{3}{c}{ Rutile } \\
\cline { 2 - 7 } & $\mathrm{a}$ & $\mathrm{b}$ & $\mathrm{z}$ & $\mathrm{a}$ & $\mathrm{b}$ & $\mathrm{u}$ \\
\hline LDA [15] & 3.751 & 9.524 & 0.2072 & 4.560 & 2.931 & 0.3039 \\
PBE [17] & 3.805 & 9.772 & 0.2057 & 4.652 & 2.976 & 0.3051 \\
Wu-Cohen [19] & 3.780 & 9.637 & 0.2066 & 4.605 & 2.995 & 0.3048 \\
PBEsol [20] & 3.779 & 9.644 & 0.2064 & 4.606 & 2.952 & 0.3048 \\
Neutron diff. [53] & 3.785 & 9.514 & 0.20806 & 4.594 & 2.959 & 0.30478 \\
Neutron diff. [54] & 3.785 & 9.512 & 0.20814 & 4.593 & 2.959 & 0.30476 \\
\hline \hline
\end{tabular}

\subsubsection{Conclusions}

In this work, first principles calculations using the LAPW+LO method for the energy ordering of rutile and anatase, the two mostly used polymorphs of $\mathrm{TiO}_{2}$, with various standard approximate density functionals have been reported. This method avoids most approximations in the treatment of the core states, such as the frozen core approximation or neglect of relativity, and also uses highly accurate basis sets and representations of the charge density and potential. Careful converge tests were done. Anatase was always found to be more stable than rutile. This is in accord with reported DQMC calculations [47]. The PBE functional, generally regarded as particularly reliable, gives the largest energy difference. 
It can be observed from the current results and prior work that more accurate methods (over LDA) e.g.: use of GGA functionals [44, 43, 50, 56, 57, 58] as well as hybrid functionals (like B3LYP and PBE0) [50] do not resolve the conundrum where anatase is obtained as the ground state, in disagreement with experiment, the details of which have been summarized in Table 3.3. The results reported here clearly show that the discrepancy is not explained by the use of pseudopotentials or other core electron related approximation, as made in several of the previous studies.

These results imply that reinvestigation of the experimental ground state may be of value, perhaps especially in relation to the extrapolation of the high-temperature enthalpy data to $0 \mathrm{~K}$. In this regard, Trail et al. [47] suggested that anharmonic effects associated with soft phonon modes may be important in stabilizing rutile especially at elevated temperature.

As of writing this thesis, a recent study reported that this well known dichotomy of the energy ordering of $\mathrm{TiO}_{2}$ polymorphs is similar to the energy ordering of two other cases of compounds, namely for the polymorphs of $\mathrm{MnO}_{2}$ and structural phase stabilities of iron disulfide, $\mathrm{FeS}_{2}$. In their study Patra et al. [60] suggested this as a case where popular and nonempirical semilocal exchange correlation functionals fail to correctly describe their energetics. They found out that the recently proposed metaGGA functional MGGAC can predict the correct ground state of these compounds along with a quantitative agreement of few of their other properties with experimental results. 
Table 3.3: Previously known stability order $\left(\Delta E=E_{\text {anatase }}-\mathrm{E}_{\text {rutile }}\right.$ in $\left.\mathrm{eV}\right)$ using various DFT methods. Various types include plane-wave (PW) or linear combination of atomic orbitals (LCAO) basis using pseudopotentials (PP) mostly. For LCAO all-electron method, a triple valence all-electron (TVAE) modification was used with d-symmetry polarization either on $\mathrm{O}$ (denoted by $\mathrm{TVAE}^{*}$ ) or on both $\mathrm{O}$ and $\mathrm{Ti}$ (denoted by TVAE** ${ }^{*}{ }^{\dagger}$ Most accurate basis set reported.

\begin{tabular}{|c|c|c|c|}
\hline \multicolumn{2}{|c|}{ Literature } & \multicolumn{2}{|c|}{$\Delta E$ (XC Functional) } \\
\hline References & Methods/Basis used & LDA & GGA \\
\hline Milman[43] & Kleinman-Bylander PP, PW & 0.033 (CAPZ) [21] & $-0.061(\mathrm{PBE})[17]$ \\
\hline Jing-Xin et al. [59] & Norm-conserving PP, PW & $-0.030(\mathrm{VWN})[22]$ & - \\
\hline Ma et al. (PW) [56] & Ultrasoft PP, PW & - & -0.057 (PW91) [15] \\
\hline Lazzeri et al. (PW) [57] & Ultrasoft PP, PW & $-0.02(\mathrm{CAPZ})[21]$ & -0.10 (PBE) [17] \\
\hline \multirow{2}{*}{ Muscat et al. (LCAO) [44] } & TVAE* (LCAO) & 0.003 (CAPZ) [21] & $-0.056(\mathrm{PBE})[17]$ \\
\hline & $\mathrm{TVAE}^{* *}(\mathrm{LCAO})$ & -0.019 (CAPZ) [21] & $-0.078(\mathrm{PBE})[17]$ \\
\hline \multirow{2}{*}{ Labat et al. [50] } & Gaussian $^{\dagger}$ (All-electron) & $-0.016(\mathrm{VWN})[22]$ & $-0.158(\mathrm{PBE})[17]$ \\
\hline & PAW-PP & $-0.048(\mathrm{VWN})[22]$ & $-0.182(\mathrm{PBE})[17]$ \\
\hline
\end{tabular}




\section{2 $\mathrm{NaSbSe}_{2}$ : A study of its thermoelectric prop- erties}

\subsubsection{Introduction}

Chalcogenide semiconductors have attracted research attention from several decades. They display an uncommon array of physical phenomena that range from interesting electronic, thermal and optical properties to novel forms of superconductivity and magnetism ([61] and references therein). When compared to binary semiconductors, this class of compounds offer more opportunities to tune and even engineer key properties $[62,63,64,65]$. A certain class of these semiconductors represented by $A B X_{2}$ can be formed with an alkali/noble metal for $A$; $\mathrm{As}$, $\mathrm{Sb}$ or $\mathrm{Bi}$ for $B$ and any of the chalcogens for $X$. Cation ordering can strongly affect properties for these compounds. Recently, ordered $\mathrm{NaSbSe}_{2}$ was reported experimentally and shown to be a promis-

ing photovoltaic material with a band gap substantially larger than the effectively semimetallic disordered phase [66]. The favorable charge carrier collection observed in this ordered material suggests that this ordered phase of $\mathrm{NaSbSe}_{2}$ might also have other useful electronic applications. Here we investigated this material in the context of thermoelectrics (TEs) [67, 68, 69].

$\mathrm{NaSbSe}_{2}$ has been long known [70, 71], but only in the disordered rock salt form which has been found to have an inadequate gap for a TE material. However, in their recent work Aragaw et al. [66], starting with its sulfide analogue: $\mathrm{NaSbS}_{2}$ precursor, were able to synthesize $\mathrm{NaSbSe}_{2}$ nanocrystals in its monoclinic structure by an ionexchange method and characterized it as a good solar absorber. The related sulfide $\mathrm{NaSbS}_{2}$ has a high dielectric constant due to enhanced Born effective charges. This 
reduces carrier scattering and helps it to be a good material for solar cell and other optoelectronic applications [72, 73].

\subsubsection{Computational details}

We performed first principles calculations on $\mathrm{NaSbSe}_{2}$ with the monoclinic (space group 15: C2/c) structure[66] to investigate its potential applications as a TE material. Our results were obtained using the general potential linearized augmented plane wave (LAPW) method [6] as implemented in the WIEN2k code [5]. We used the generalized gradient approximation by Perdew, Burke and Ernzerhof (PBE) [17] to determine the ground state with a well-converged $12 \times 10 \times 12$ sampling for the Brillouin zone integration. Electronic structure and transport quantities were calculated using a k-mesh twice as dense in each direction. The modified Becke-Johnson (mBJ) potential of Tran and Blaha [23] which is known to improve the band gaps significantly for a wide range of materials $[23,74,75,76]$ was used for this purpose. The spin-orbit interaction was included in these calculations. This was done using the second variational method implemented in WIEN2k.

The calculations were done using LAPW sphere radii of 2.45 Bohr for all atoms. The basis size was set by a plane-wave cutoff, $K_{\max }$ determined by $R_{\min } K_{\max }=9.0$ (with $R_{\min }=R=2.45 \mathrm{bohr}$ ). Local orbitals were included for semicore states. The structure was taken from variable-cell relaxation calculation results as reported by Aragaw et al [66]. We employed the constant scattering time approximation (CSTA), as implemented in the BoltzTraP code [7], for calculating TE transport properties. Within the CSTA, the thermopower $(S)$ can be obtained as a function of temperature 
and doping concentration directly from the band structure without any adjustable parameters. We also investigated the Born effective charges and the dielectric tensor within density functional perturbation theory (DFPT) as implemented in the vasp code using PAW pseudopotentials [77]. We used the PBE functional for these response calculations. This is because the mBJ potential is not a total energy functional and hence cannot be used to calculate responses.

\subsubsection{Results and discussion}

$\mathrm{NaSbSe}_{2}$, like other similar compounds e.g. $\mathrm{NaSbTe}_{2}$ and $\mathrm{NaBiTe}_{2}$, is known [71, 78, 79] to exist in a NaCl-like cubic structure, which is cation disordered. However, an ordered monoclinic structure was recently reported based on an ion exchange process [66]. Figure 3.1 shows this structure. It is monoclinic with space group C2/c (no. 15) with $a=8.433 \AA, b=7.230$ Ånd $c=8.716$ Aand monoclinic angle $\gamma \sim 57^{\circ}$. Cartesian coordinate axes $y$ and $z$ have been chosen along $b$ and $c$ respectively and the orthogonal $\mathrm{x}$ axis accordingly for the discussion below.

The structure is formed of $\mathrm{Na}$ ions and $\mathrm{Se}-\mathrm{Sb}-\mathrm{Se}$ units. These units have a Se-Sb-Se angle of $\sim 99.5^{\circ}$ and are placed such that the Sb lone pair is directed towards Na. This forms layers stacked along the slanted b-axis. Each unit cell contains two of these layers but it should be noted that these units are oppositely oriented.

Standard density functionals such as the PBE functional are designed for accurate energy prediction [52] but underestimate the band gaps of semiconductors. Using 


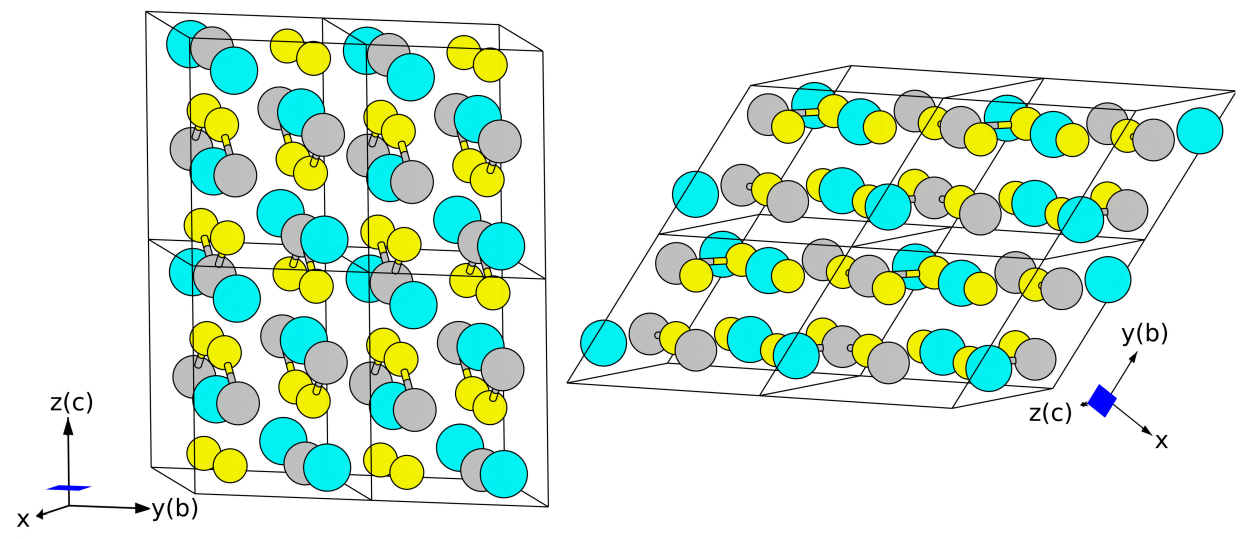

Figure 3.1: Layered structure of ordered $\mathrm{NaSbSe}_{2}$. The yellow spheres are Se, the blue are $\mathrm{Na}$ and the gray ones are $\mathrm{Sb}$ atoms. There are 2 formula units in the unit cell. On left the Se-Sb-Se units are shown. These along with Na atoms make up the layers. Individual layers are stacked as shown on the right.

PBE, a gap of $0.553 \mathrm{eV}$ was obtained. The mBJ potential, designed for gaps and energies, along with spin-orbit coupling yields an indirect gap of $0.843 \mathrm{eV}$. This band gap for this semiconductor is sufficient to avoid any major bipolar conduction effects; an essential property of any good doped TE. The bipolar effect as shown by narrow band gap TE materials is phenomenon where at sufficiently high temperatures, the minority carriers in the semiconductor are excited across the band gap. This additional conduction deteriorates its performance due to the undesirable lowering of the Seebeck coefficient. The disordered cubic lattice is known to melt at 1013 $\mathrm{K}$ [80]. Upon heating, at around $753 \mathrm{~K}$, cation disorder is reported to be induced in the monoclinic structure [66]. Here we consider the temperature range up to $600 \mathrm{~K}$.

The electronic density of states (DOS) and their projections onto the LAPW spheres are shown in figure 3.2. Na is fully ionized and does not contribute to the va- 
lence bands significantly. The valence bands are derived from Se $p$ states as expected for the $\mathrm{Se}^{2-}$ ions. In the same interval down from $-5 \mathrm{eV}$ to $0 \mathrm{eV}$, with respect to the valence band maximum (VBM), there is substantial Sb-5p orbital character concentrated around the bottom edge of this range. In the conduction band, similar Se and $\mathrm{Sb} p$ orbital contributions are found. This indicates a high degree of hybridization between the Se and Sb states i.e. a significant amount of cross-gap hybridization between Se- $4 p$ and Sb-5p states. We also note a small peak close to the VBM which has antibonding Sb- $4 s$ character. Whereas the main Sb- $4 s$ states lie in a peak around $7 \mathrm{eV}$ depth below VBM.

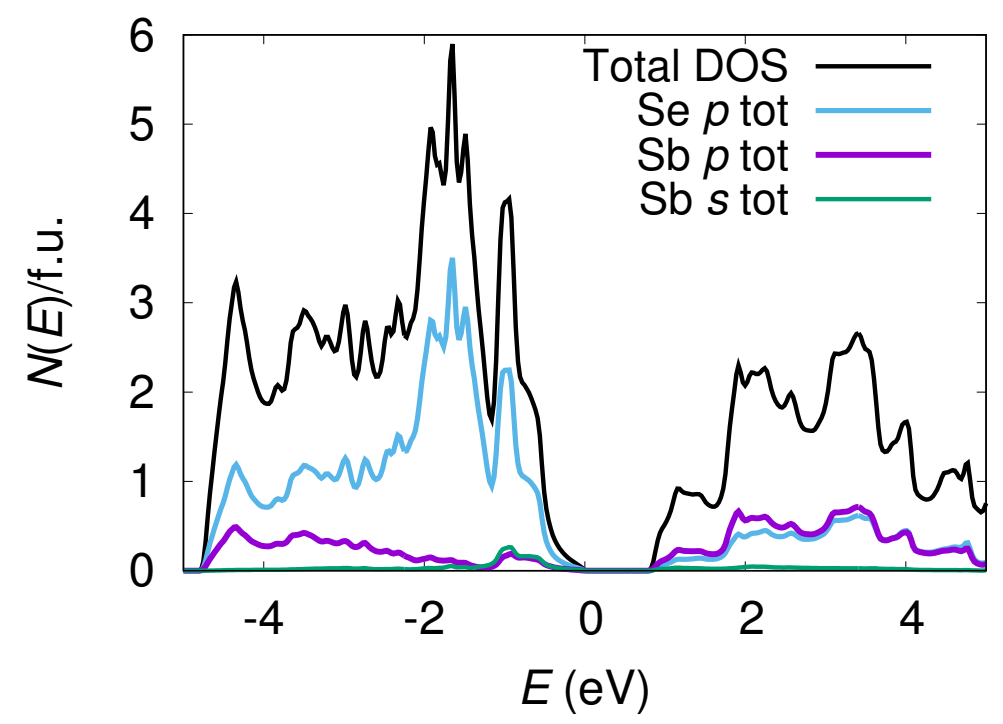

Figure 3.2: DOS and its projections onto the LAPW spheres shown on a per formula unit basis.

This cross-gap hybridization may give rise to enhanced Born effective charges as in the case of many oxides [81]. The Born effective charge tensor is a response function $\left(Z_{\kappa, \alpha \beta}^{*}\right)$ for an atom $\kappa$ that is defined as the coefficient of proportionality at linear 
order between its change in its macroscopic polarization $\left(\mathcal{P}_{\beta}\right)$ per unit cell in direction $\beta$ caused by an atomic displacement in the direction $\alpha\left(\tau_{\alpha}\right)$ under the condition of zero external electric field $(\mathbf{q}=0)$ as given below in equation 3.1. Since polarization can be defined as a first derivative of energy with respect to the macroscopic electric field, this can be utilized to transform this in terms of a linear relation between the force and electric field $\epsilon_{\beta}$ as [83]:

$$
Z_{\kappa, \alpha \beta}^{*}=\left.\Omega_{0} \frac{\partial \mathcal{P}_{\beta}}{\partial \tau_{\kappa \alpha}}\right|_{\mathbf{q}=0}=-\left.\frac{\partial^{2} E_{t o t}}{\partial \epsilon_{\beta} \partial \tau_{\kappa \alpha}}\right|_{\mathbf{q}=0}=\frac{\partial F_{\kappa, \alpha}}{\partial \epsilon_{\beta}}
$$

We performed DFPT calculations using the PBE functional. The resulting Born effective charge tensor and the macroscopic dielectric tensor are given in tables 3.4 and 3.5 .

Table 3.4: Calculated Born effective charge tensor for monoclinic $\mathrm{NaSbSe}_{2}$. For $\mathrm{Se}^{*}$, the dual Born charges indicate the relatively opposite alignment of each Se atom.

\begin{tabular}{cccccccccc}
\hline \hline & $x x$ & $x y$ & $x z$ & $y x$ & $y y$ & $y z$ & $z x$ & $z y$ & $z z$ \\
\hline $\mathrm{Na}$ & 1.39 & -0.31 & 0.00 & -0.26 & 1.32 & 0.00 & 0.00 & 0.00 & 1.18 \\
$\mathrm{Sb}$ & 2.50 & 0.09 & 0.00 & -0.05 & 6.01 & 0.00 & 0.00 & 0.00 & 2.18 \\
$\mathrm{Se}^{*}$ & -1.95 & 0.11 & \pm 0.91 & 0.16 & -3.66 & \pm 1.15 & \pm 0.79 & \pm 0.48 & -1.68 \\
\hline \hline
\end{tabular}

Table 3.5: Dielectric tensor of monoclinic NaSbSe ${ }_{2} . \epsilon_{\infty}$ is the electronic whereas $\epsilon_{\mathrm{ph}}$ is the lattice contribution to the total macroscopic dielectric tensor: $\epsilon$.

\begin{tabular}{ccccc}
\hline \hline & $x x$ & $x y$ & $y y$ & $z z$ \\
\hline$\epsilon_{\infty, \mathrm{ij}}$ & 9.2 & 1.4 & 13.1 & 9.6 \\
$\epsilon_{\mathrm{ph}, \mathrm{ij}}$ & 9.4 & -14.1 & 60.1 & 3.3 \\
$\epsilon_{i j}$ & 18.6 & -12.7 & 73.2 & 12.9 \\
\hline \hline
\end{tabular}


The Born effective charges for Se and Sb are quite different from the nominal valence and are enhanced in the $y$ direction. This is also reflected in table 3.5 for the dielectric constant where a high value is found along the $y$ direction. The lattice part has a much higher contribution along the same direction reflecting the origin in the high $y$ direction Born effective charges. This large dielectric response can be beneficial in screening ionized impurities [82]. Defect tolerance due to charge screening has been shown to be beneficial for carrier transport in various other semiconductors [84].

Now we discuss transport properties in relation to the TE performance $Z T=$ $\sigma S^{2} T / \kappa$. High $Z T$ demands a high electrical conductivity $(\sigma)$ simultaneously with a high Seebeck coefficient $(S)$. $\mathrm{NaSbSe}_{2}$ belongs to a chalcogenide chemistry that is known to be associated with very low thermal conductivity, $\kappa$ related to its bonding characteristics [85]. Here we focus on the electronic aspect of ZT. Being countercorrelated, increasing the carrier concentration generally increases $\sigma$ but at the same time also reduces $S$. This poses one of the central challenges in finding high performance TE materials.

In attempts to address this, it has been shown that materials with certain complex and/or non-parabolic band structure features, for example carrier-pocket anisotropy [86, 87, 88], reduced dimensionality [89, 90, 91, 92, 93], exploitable anisotropies [94, 95] and band degeneracies due to convergence $[96,97,98]$ may result in decoupling of $\sigma$ and $S$. Such decoupling is important for realizing high thermoelectric performance [68, 99, 100]. Essentially, these electronic band structure features are all significant deviations from the isotropic single parabolic band (SPB) model and have been used 
to find high $Z T$ TEs. These features in some cases can be visually identified in carrier pocket shapes. Energy isosurfaces are shown in figure 3.3.

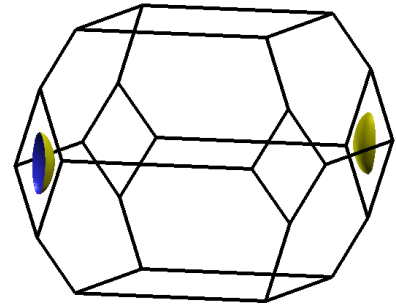

VBM - $0.05 \mathrm{eV}$

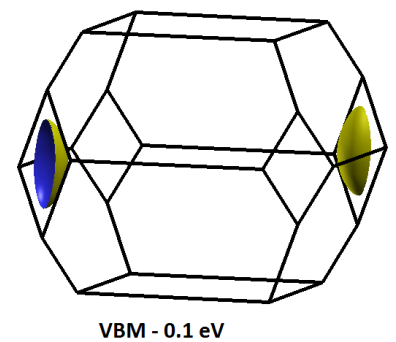

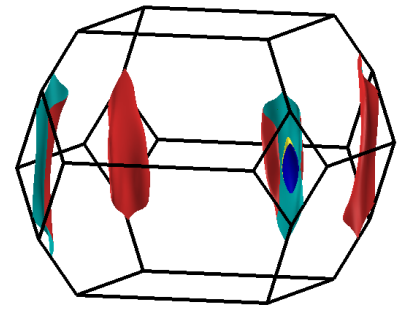

$\mathrm{CBM}+0.05 \mathrm{eV}$

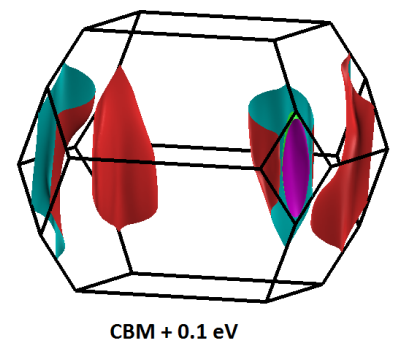

Figure 3.3: Isoenergy surfaces at $0.05 \mathrm{eV}$ and $0.1 \mathrm{eV}$ below VBM ( $p$-type, left panel) and above conduction band minima $[\mathrm{CBM}]$ ( $n$-type, right panel) showing various carrier-pockets.

The isoenergy surfaces in figure 3.3 are complex in nature and deviate substantially from ideal ellipsoidal shapes as described by the SPB model and additionally, are clearly anisotropic. For $p$-type, they are flattened ellipsoids on the Brillouin zone faces. Whereas for $n$-type, sheets are formed at the zone boundaries which upon increasing the doping concentration, connect in order to form approximate cylinders that run along $k_{z}$. Contrary to the $p$-type, this elongation represents an increase in the transport effective mass along this direction. This direction corresponds to the direction of inter-planar transport in the real-space structure. 
We now discuss the transport quantities more quantitatively. In figure 3.4, the Seebeck coefficients are plotted in each of the three directions as a function of carrier concentration at $T=300,400$ and $600 \mathrm{~K}$. The reduced electrical conductivities $(\sigma / \tau)$ are also shown.
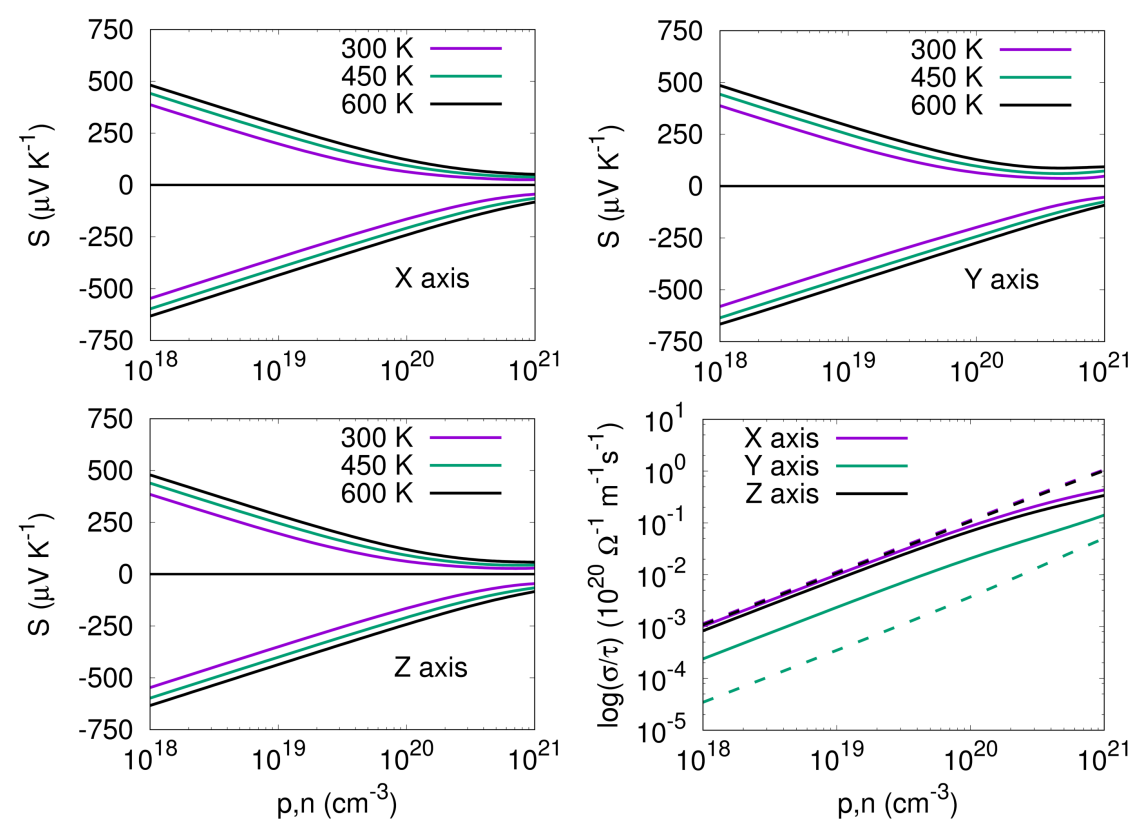

Figure 3.4: Seebeck coefficients (in $\mu \mathrm{VK}^{-1}$ ) at various temperatures in all three directions as a function of either doping concentration. [Bottom right] reduced conductivity (in $10^{20} \Omega^{-1} m^{-1} s^{-1}$ ) on a log-log scale for both $p$ - (solid lines) and $n$-type (dashed lines) doping.

We find the $n$-type Seebeck coefficient to be larger than the $p$-type, almost by a factor of two. This reflects the steeper onset of the $n$-type DOS [92]. In the $10^{19}$ $10^{20} \mathrm{~cm}^{-3}$ doping range it is as high as $300 \mu \mathrm{V} \mathrm{K}^{-1}$ for the $n$-type. For TEs where the thermal conductivity is typically approximated as $\kappa=\kappa_{e}+\kappa_{l}$, the sum of electrical 
and lattice thermal conductivity contributions respectively, one can apply the Wiedemann-Franz law, $\kappa=L \sigma T$. With this and the standard value of $L=L_{0}$, it can be shown that one has to have $S \gtrsim 160 \mu V K^{-1}$ in order to obtain $Z=S^{2} \sigma / \kappa$ over unity in the (ideal) limit of vanishing $\kappa_{l}$. Despite being almost isotropic, $S$ has slightly larger values along the $y$-direction in either doping types. The effect is stronger for $n$-type where the carrier pockets become open cylinders at comparatively low energy relative to the band edge. In the lower right panel of figure 3.4, the reduced electrical conductivity $(\sigma / \tau)$ shows similar trends. This significant anisotropy in the conduction band masses results into about an order of magnitude difference between the in-plane $(x$ and $z$ ) and out-of-plane $(y)$ direction conductivity values.

The reduced power factor (RPF) defined as $S^{2} \sigma / \tau$, captures the combined effect of both $\sigma$ and $S$ on the TE transport and is shown in figure 3.5. One may note the large difference in the RPF in the $x, z$ direction from the $y$ direction arising from its lower conductivity. This difference is about 20 times larger for $n$-doped case reflecting the flatter anisotropic energy isosurface shapes.

As mentioned, there are a number of band structure features that can decouple $\sigma$ and $S$ in doped semiconductors. Such band structure complexities relevant to TEs can be quantified using an EFF defined as: $(\sigma / \tau) S^{2} / N^{2 / 3}$ where $N$ is the volumetric density of states $[65,92]$. This function is low when either conductivity or thermopower is low, and is high when conductivity and thermopower are both high- a combination that does not generally occur for a single isotropic parabolic band. As mentioned, the EFF is a temperature and doping level dependent function based on 

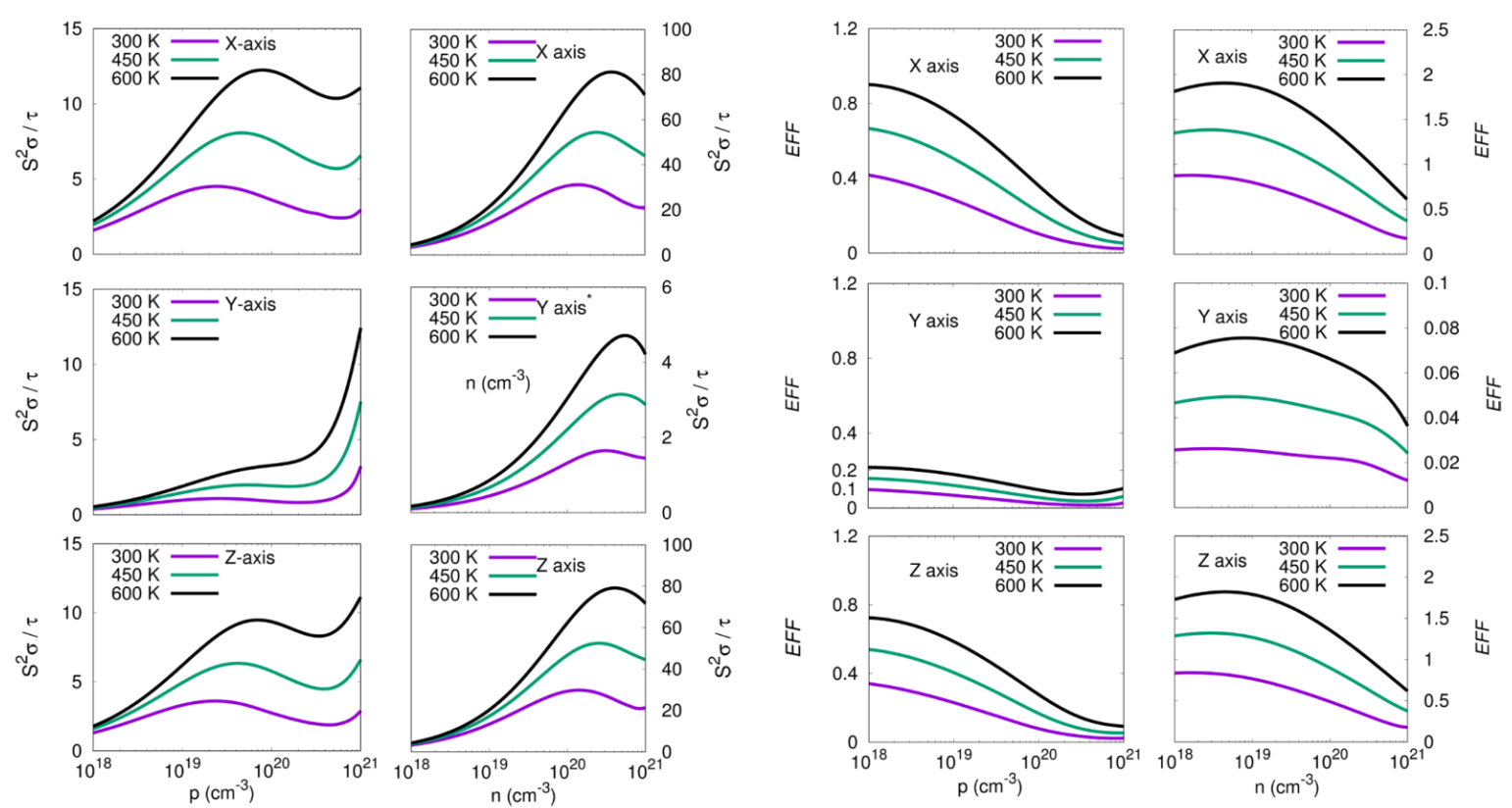

Figure 3.5: [Left Panel] Reduced power factor: $S^{2} \sigma / \tau$ (in $10^{10} \mathrm{Wm}^{-1} \mathrm{~K}^{-2} \mathrm{~s}^{-1}$ ) at various temperatures in all three directions as a function of both $p$ - (left half) and $n$-type (right half) doping concentration. Note the difference in scales, especially for y direction of $n$-type. [Right Panel] Calculated EFF (in $10^{-19} \mathrm{~W}^{5 / 3} \mathrm{~ms}^{-1 / 3} \mathrm{~K}^{-2}$ ) in all three directions for monoclinic $\mathrm{NaSbSe}_{2}$ as a function of doping concentration at various temperatures for both $p$ - and $n$-type doping. Note the similar difference in scales.

transport integrals. It characterizes the extent to which the band shape favors high $Z T$, independent of differences in scattering mechanisms or the thermal conductivity.

The EFF for $\mathrm{NaSbSe}_{2}$ is shown on right in figure 3.5. We restrict our discussion to $T=600 \mathrm{~K}$, and below, since the stability of the ordered structure is doubtful above this temperature.

As seen, on right of in figure 3.5, for $n$-type doping, the in-plane/out-of-plane anisotropy in EFF is larger than 25. This means that $n$-type $\mathrm{NaSbSe}_{2}$ will have strongly anisotropic TE properties. This is a consequence mainly of strong anisotropy 

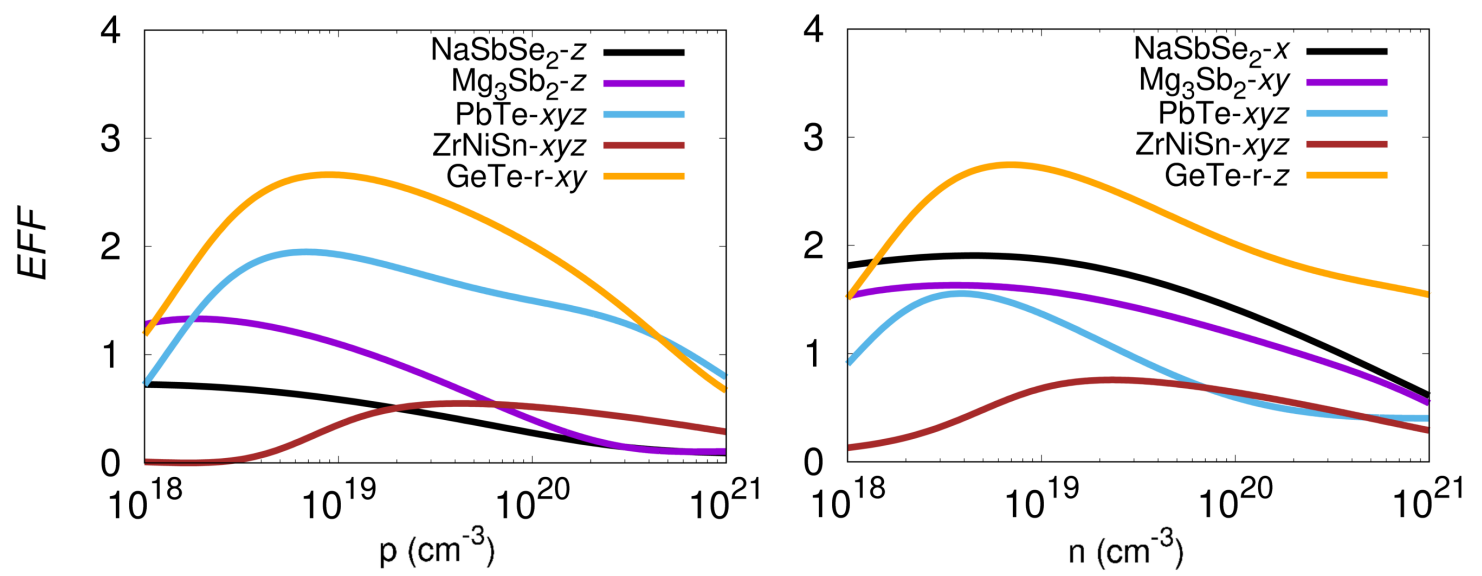

Figure 3.6: Comparison of EFF (in $10^{-19} \mathrm{~W}^{5 / 3} \mathrm{~ms}^{-1 / 3} \mathrm{~K}^{-2}$ ) calculated along direction of best performance for four other known TEs namely $\mathrm{Mg}_{3} \mathrm{Sb}_{2}, \mathrm{PbTe}, \mathrm{GeTe}$ and $\mathrm{ZrNiSn}$ with both doping types. Note that at $600 \mathrm{~K}$ we have the rhombohedral structure for the highest EFF value material GeTe.

in the conductivity, and in particular low conductivity perpendicular to the layers. In the following we focus in the in-plane directions. In figure 3.6, we present a comparison of EFF with four other established TE materials at $600 \mathrm{~K}$ in their corresponding crystal directions of best performance (i.e. highest EFF directions).

The superior TE performance of $n$-doped $\mathrm{NaSbSe}_{2}$ along the $x$-direction as indicated by calculated EFF can be noted from figure 3.6. It is next only to rhombohedral GeTe, which has comparable EFF for either doping type [65, 101]. The peak EFF for $n$-type $\mathrm{NaSbSe}_{2}$ is close to that of $n$-type $\mathrm{Mg}_{3} \mathrm{Sb}_{2}$, which is a newly discovered high performance Zintl TE, with especially favorable properties at intermediate temperature when the doping is optimized $[102,103]$. In relation to $\mathrm{PbTe}$, which is currently used in thermoelectric generators, the EFF for $n$-type $\mathrm{NaSbSe}_{2}$ is comparable for the same temepratures. Lastly, the half-Heusler compound ZrNiSn, taken as a known [104] nanocomposite TE to standardize the EFF values [65] lies lower than $n$-type 
optimally oriented $\mathrm{NaSbSe}_{2}$.

Three interesting aspects of the EFF of $n$-type $\mathrm{NaSbSe}_{2}$ are that the EFF is strongly increasing with temperature over the range shown, that the EFF is rather isotropic in-plane ( $x-z$ directions $)$, and that the EFF maximum in-plane is rather flat over a wide concentration range at fixed temperature. Specifically, the EFF changes by only $\sim 10 \%$ in the doping range $10^{18}-10^{19}$ at $600 \mathrm{~K}$ for $x$ and $z$ directions. This means that control of the exact carrier concentration is less important in this material than in many other TEs, and moreover that a single carrier concentration will be near optimal over the whole temperature range from $300 \mathrm{~K}$ to $600 \mathrm{~K}$. The in-plane near isotropy means that high performance might occur not only in suitably oriented single crystals, but also in ceramics, provided that the ceramics are highly textured for in-plane transport. The increase with temperature implies that it will be of interest to experimentally determine what temperature can be used while retaining stability of the structure, and also whether chemical modifications can be made that improve the stability.

\subsubsection{Conclusions}

To summarize, we performed first-principles electronic structure and transport calculations and determined the TE properties of newly synthesized monoclinic $\mathrm{NaSbSe}_{2}$. This material was previously known in literature as a cation disordered material where its layer ordered form has been recently synthesized in nanostructured form. Our first-principles results showed cross-gap hybridization between Se- $4 p$ and Sb- $5 p$ 
states. We also found this in conjunction to enhanced Born effective charge and high dielectric constant $(\epsilon)$. Keeping thermoelectric transport in mind, this is especially helpful in achieving defect-tolerance against ion-impurity scattering and helps increase electron mobility. A large Seebeck coefficient coupled with anisotropically high electrical conductivity features are also found through specific transport calculations using Boltzmann theory. The calculated large anisotropies in $\epsilon, S$ and $\sigma$ are in accord with its layered structure. Complex energy isosurface structure with properties favorable for the electronic part of the TE transport are also found from the study of electronic structure. Based on the calculated EFF, a metric formulated to evaluate TEs theoretically, the material shows behavior competitive to the best known TEs in this temperature range. In conclusion, $n$-type $\mathrm{NaSbSe}_{2}$ possesses electronic structure properties favorable for TE transport. Experimentally, it would be of interest to investigate doping and the resulting TE performance of this material when doped. 


\section{Chapter 4}

\section{Thermoelectrics: An insight into the Lorenz number}

In this chapter, we examined the Lorenz number $(L)$ as used in the Wiedemann-Franz relation in the context of Thermoelectrics (TEs), with a focus on one of the recurrent formulas in the literature. Based on the observed Seebeck coefficient, it is used to estimate $L$ in experimental investigation of novel TEs. We used the Boltzmann transport theory to test this formula for realistic electronic structures of a few known TEs in different scattering regimes.

We find that this expression, captures the observed variations of $L$ with satisfactory accuracy for some materials while deviating substantially for some other ones that have non-parabolic band structures. This implies caution should be exercised especially in TEs and other materials that may have complex electronic structures. 


\subsection{Introduction}

Heat conduction in materials generally involves multiple transport channels, including electronic and phononic, and these may be coupled. It is often important to separate these components to reveal the physics of heat transport. This is particularly significant in thermoelectrics (TEs), where understanding lattice thermal conductivity and mechanisms for its reduction are crucial $[105,106,107,108,109,110]$. In principle, the electronic conductivity and corresponding electronic thermal conductivity can be suppressed by magnetic field, allowing direct measurement of the lattice thermal conductivity, $\kappa_{l}$. This has been carried out for several materials including $\mathrm{Bi}_{2} \mathrm{Te}_{3}$ crystals and some other materials generally below room temperature $[109,111]$. However, the regime where the cyclotron time and relaxation time are comparable, it is not achievable in ordinary TEs at temperatures where they are used. Therefore, even though the conditions can be somewhat relaxed by appropriate fits [109], only the total thermal conductivity, $\kappa$, consisting of the sum of all these parts is normally accessible by direct measurement in ordinary TEs.

Then, empirical methods are used to separate the lattice and electronic contributions to $\kappa$. It is common to use the Wiedemann-Franz (WF) relation for this [107]. This states that $\kappa_{e}=L T \sigma$, where $\kappa_{e}$ is the electronic component of $\kappa, \sigma$ is the electronic conductivity, $T$ is the temperature and $L$ is the Lorenz number which

is often treated as a constant, $L_{0}=\frac{\pi^{2}}{3}\left(k_{B} / e\right)^{2}=2.443 \times 10^{-8}{\mathrm{~W} \Omega \mathrm{K}^{-2}}^{- \text {Flage-Larsen }}$ and Prytz [113] used specific parametrizations with a single parabolic band (SPB) model to estimate electronic transport through $L$ for different model scattering mechanisms, temperatures, chemical potential and carrier concentration. Going beyond 
this, Maciá [112] derived $L, \kappa_{e}$ and other transport coefficients analytically in combination to phenomenological parameters for complex metallic alloys and obtained corrections to the WF relation in accord with experiments.

It must be noted that the WF relation as stated previously holds under the ideal conditions of the degenerate doping limit, with additional assumptions such as elastic scattering. These generally hold for metals and heavily doped semiconductors at temperatures where electronic heat transport dominates, the case where $L$ converges to $L_{0}$. However, in practice, semiconductor TEs often lie in between the heavily doped degenerate limit and the lightly doped non-degenerate limit, and here, $L$ may deviate significantly from $L_{0}$ [114]. This also may depend on the associated electronic scattering mechanisms, its temperature dependence and other factors $[112,115,116]$.

In an attempt to estimate $L$ more accurately, Kim et al. [117], using experimental data, proposed an approximate equation by which one can estimate $L$ directly from Seebeck coefficient $(S)$ measurements. This enabled experimentalists to quickly estimate $L$ at any temperature without having to depend on less accessible quantities e.g. Hall mobility and so on. This expression has become very widely used and is the subject of the present work [118, 119, 120, 121, 122]. Assuming a parabolic band model with acoustic phonon scattering, its (simplified) form as used is:

$$
L=1.5+\exp \left[-\frac{|S|}{116}\right]
$$

This equation is intended to provide a better approximation to $L\left(\right.$ in $\left.10^{-8}{\mathrm{~W} \Omega \mathrm{K}^{-2}}^{2}\right)$ obtained from measured $S$ (in $\mu V K^{-1}$ ) as compared with treating it as the constant 
$L_{0}$

Previously, Wang and co-workers [123], explored the factors affecting its generality for $\mathrm{Si}$ and $\mathrm{Bi}_{2} \mathrm{Te}_{3}$ via the Landauer approach and found that $L$ can be much smaller than the expected non-degenerate limit $L=2\left(k_{B} / \mathrm{e}\right)^{2}=1.48 \times 10^{-8}{\mathrm{~W} \Omega \mathrm{K}^{-2}}^{2}$. Here, we studied the accuracy of this expression by comparing it with first-principles results obtained for a few known TEs as well as numerically solving the Boltzmann transport equations for the SPB model, which is the origin of this formula.

The semiclassical theory of electronic transport or the Boltzmann transport equation (BTE) under the relaxation time approximation results in the following equations (in their scalar form):

$$
\begin{aligned}
\sigma & =\int \sigma(E) d E \\
S & =-\frac{1}{e T} \frac{\int\left(E-E_{F}\right) \sigma(E) d E}{\int \sigma(E) d E} \\
\kappa_{e} & =\frac{1}{e^{2} T} \int\left(E-E_{F}\right)^{2} \sigma(E) d E
\end{aligned}
$$

where

$$
\sigma(E)=e^{2} \Xi(E)\left(-\frac{\partial f_{0}}{\partial E}\right)
$$

Here $f_{0}$ is the Fermi function and $\tau$ is the relaxation time and $e$ is the magnitude of electronic charge. The integrals are over the entire energy range. The transport 
function $\Xi(E)$ is derived from the band structure, which in its tensor form is

$$
\Xi_{\mathrm{ij}}(E)=\int \frac{2 d \vec{k}}{(2 \pi)^{3}} v_{\mathrm{i}}(\vec{k}) v_{\mathrm{j}}(\vec{k}) \tau(E) \delta[E-E(\vec{k})]
$$

with

$$
\vec{v}(\vec{k})=\frac{1}{\hbar} \nabla_{\vec{k}} E(\vec{k})
$$

such that

$$
\Xi_{\mathrm{SPB}}(E)=N(E) v(E)^{2} \tau(E)
$$

Equation 4.2d states the standard definition of $\kappa$ [124]. However, for materials that are not metals, the presence of a temperature gradient $(\nabla T)$ induces an electric field and vice versa $[125,126]$. This is the source of thermoelectric effects. This leads to an additional contribution to the open circuit thermal conductivity:

$$
\kappa_{\text {open }}=\kappa_{e}-T \sigma S^{2}
$$

Although these equations hold good for materials with a general band structure, it is also relevant to study the analytical results applicable for the SPB model. In this ideal case, one can solve BTE to obtain both $S$ as well as $L$ directly in terms of a scattering law exponent $(\lambda)$ and the relevant chemical potential ( $\eta$, depending on the carrier concentration). These analytic results (using the expression in equation 
4.4 can be summarized as:

$$
\begin{gathered}
L=\left(\frac{k_{B}}{e}\right)^{2} \frac{(1+\lambda)(3+\lambda) F_{\lambda}(\eta) F_{\lambda+2}(\eta)-(2+\lambda)^{2} F_{\lambda+1}^{2}(\eta)}{(1+\lambda)^{2} F_{\lambda}^{2}(\eta)} \\
S=\frac{k_{B}}{e}\left(\frac{(2+\lambda) F_{\lambda+1}(\eta)}{(1+\lambda) F_{\lambda}(\eta)}-\eta\right)
\end{gathered}
$$

Here, $F_{\lambda}(\eta)$ is the generalized Fermi integral over the entire energy range and is given by:

$$
F_{j}(\eta)=\int \frac{E^{j} d E}{1+\exp \left(\frac{E-\eta}{k_{B} T}\right)}
$$

Using these above expressions, we define the $L$ in two different ways as [127]:

$$
\begin{gathered}
L_{e}=\frac{k_{e}}{\sigma T} \\
L_{\text {open }}=\frac{k_{\text {open }}}{\sigma T}
\end{gathered}
$$

We use both of these expressions and investigate the variations in it from the degenerate value $L_{0}$ in different materials. We find, although in some cases equation 4.1 is close to the exact result, important deviations are found in others, including practically relevant cases for TE research. 


\subsection{Computational methods}

We investigated the electronic structure of four known TEs viz. $\mathrm{Mg}_{3} \mathrm{Sb}_{2}, \mathrm{PbTe}$, $\mathrm{SrTiO}_{3}$ and $\mathrm{ZrNiSn}$ via density functional theory calculations with the all-electron linearized augmented planewave (LAPW) method [6] as implemented in the WIEN2k code [5]. We used experimental lattice parameters as given in table 4.1. Where applicable, we relaxed the internal atomic coordinates.

Table 4.1: Used lattice paramaters from experiments with relaxed internal coordinates (in case of $\mathrm{Mg}_{3} \mathrm{Sb}_{2}$ )

\begin{tabular}{|c|c|c|c|c|c|c|c|}
\hline \multirow{2}{*}{ Material } & \multirow{2}{*}{ Space Group } & \multirow{2}{*}{ a $(\AA)$} & \multirow{2}{*}{ c $(\AA)$} & \multirow{2}{*}{ Sp. } & \multicolumn{3}{|c|}{ Internal coor. } \\
\hline & & & & & $\mathrm{x}$ & $\mathrm{y}$ & z \\
\hline \multirow{3}{*}{$\mathrm{Mg}_{3} \mathrm{Sb}_{2}[128]$} & \multirow{3}{*}{$\mathrm{P} \overline{3} \mathrm{~m} 1,164$} & \multirow{3}{*}{4.559} & \multirow{3}{*}{7.243} & Mg1 & 0 & 0 & 0 \\
\hline & & & & Mg2 & $1 / 3$ & $2 / 3$ & 0.6315 \\
\hline & & & & $\mathrm{Sb}$ & $1 / 3$ & $2 / 3$ & 0.2247 \\
\hline \multirow{3}{*}{$\mathrm{PbTe}[129]$} & \multirow{3}{*}{$\mathrm{Fm} \overline{3} \mathrm{~m}, 225$} & \multirow{3}{*}{6.454} & \multirow{3}{*}{6.454} & $\mathrm{~Pb}$ & 0 & 0 & 0 \\
\hline & & & & $\mathrm{Te}$ & $1 / 2$ & $1 / 2$ & $1 / 2$ \\
\hline & & & & $\mathrm{Sr}$ & 0 & 0 & 0 \\
\hline \multirow{3}{*}{$\mathrm{SrTiO}_{3}[130]$} & \multirow[t]{3}{*}{$\operatorname{Pm} \overline{3} \mathrm{~m}, 221$} & \multirow[t]{3}{*}{3.906} & \multirow[t]{3}{*}{3.906} & $\mathrm{Ti}$ & $1 / 2$ & $1 / 2$ & $1 / 2$ \\
\hline & & & & $\mathrm{O}$ & $1 / 2$ & 0 & $1 / 2$ \\
\hline & & & & $\mathrm{Zr}$ & 0 & 0 & 0 \\
\hline \multirow{2}{*}{ ZrNiSn[131] } & \multirow{2}{*}{$\mathrm{F} \overline{4} 3 \mathrm{~m}, 216$} & \multirow{2}{*}{6.115} & \multirow[t]{2}{*}{6.115} & $\mathrm{Ni}$ & $1 / 4$ & $1 / 4$ & $1 / 4$ \\
\hline & & & & $\mathrm{Sn}$ & $1 / 2$ & $1 / 2$ & $1 / 2$ \\
\hline
\end{tabular}

We used the Perdew-Burke-Ernzerhof functional to relax the atomic coordinates of $\mathrm{Mg}_{3} \mathrm{Sb}_{2}$ [17]. We then used the modified Becke-Johnson (mBJ) potential to calculate the electronic and transport properties [23]. The mBJ functional, as discussed in the previous chapter, is known to yield improved band gaps relative to experiment for a wide variety of simple semiconductors and insulators [23, 74], including $\mathrm{Mg}_{3} \mathrm{Sb}_{2}$ [132]. Spin-orbit coupling was included for all calculations except for the determina- 
tion of internal atomic coordinates. In particular, spin orbit was included for all the electronic structures and transport properties that are presented here. The LAPW sphere radii $\left(R_{\mathrm{MT}}\right)$ of the individual atoms were fixed at 2.4 Bohr except that 1.8 Bohr was used for $\mathrm{Ti}$ and $\mathrm{O}$ and $2.2 \mathrm{Bohr}$ for $\mathrm{Sr}$ in $\mathrm{SrTiO}_{3}$. $R_{\mathrm{MT}}$ for $\mathrm{Sb}$ in $\mathrm{Mg}_{3} \mathrm{Sb}_{2}$ was fixed at 2.6 Bohr. The plane wave sector basis set was constructed by choosing $R_{\min } \mathrm{K}_{\max }=9$ for all the calculations. Brillouin zone integrations were carried out on a k-mesh of $20 \times 20 \times 11$ for rhombohedral $\mathrm{Mg}_{3} \mathrm{Sb}_{2}$ and $20 \times 20 \times 20$ for the other TEs. For the transport calculations, the number of $k$-points was further doubled in each $k$-direction.

We solved the Boltzmann transport equations with the constant scattering time approximation (CSTA) as implemented in the BoltzTraP code [7]. We also used the acoustic phonon scattering (APS) time approximation by modifying the code. We calculated $S$ and $L$ in both of these cases. The APS was implemented by setting $\tau_{\mathrm{APS}}(E)$ $\propto 1 / \tau N(E)$, using $N(E)$ as the electronic density of states (DOS) [123, 126, 133]. This is the APS formula for a general band structure and reduces to the APS scattering exponent, $\tau^{-1} \sim E^{1 / 2}$ for a single parabolic band, where $\mathrm{E}$ is the energy from the band edge. We also solved equation 4.2 for the SPB model numerically with unit effective mass under both CSTA and APS approximations independent of equation 4.5 and found agreement with the formula as expected. It should be emphasized that carrier scattering in thermoelectric materials is generally complex involving both multiple scattering mechanisms and sample dependence. Nonetheless, our results, which show substantial deviations from the formula for TEs with complex band structures, do illustrate the limitations of this formula. 


\subsection{Results and discussion}

The band gaps obtained using the mBJ potential were $0.58 \mathrm{eV}, 0.33 \mathrm{eV}, 2.71 \mathrm{eV}$ and $0.46 \mathrm{eV}$ for $\mathrm{Mg}_{3} \mathrm{Sb}_{2}, \mathrm{PbTe}, \mathrm{SrTiO}_{3}$ and $\mathrm{ZrNiSn}$, respectively. As mentioned, the actual scattering mechanisms in materials under experimental investigation are generally difficult to determine and may vary [103], so here, we examine the accuracy of the proposed estimate of $L$ as given by equation 4.1 in both the CSTA and APS regimes. Results obtained by numerically solving equation 4.2 or the SPB model together with those from equation 4.1 were included for reference in each case.

In CSTA, $\tau(E)$ comes out of the integral as a constant for each of the coefficients. However, in the APS case, recalling the DOS substitution for $\tau(E)$ as shown

previously one may also obtain both $L$ and $S$. In literature, Wang et al. [123] and McKinney et al. [126] used this energy dependent scattering rate to explicitly include APS.

In figure 4.1, one may note that $S$ is lower in the APS than in the CSTA. This is due to the DOS dependent relaxation time as mentioned earlier. $p$-type PbTe can be seen to show the largest such reduction of $S$ among the two scattering time approximations. This is related to the highly non-parabolic band structure.

We used the standard expression from equation $4.7 \mathrm{~b}$ and calculated $L$ for both 

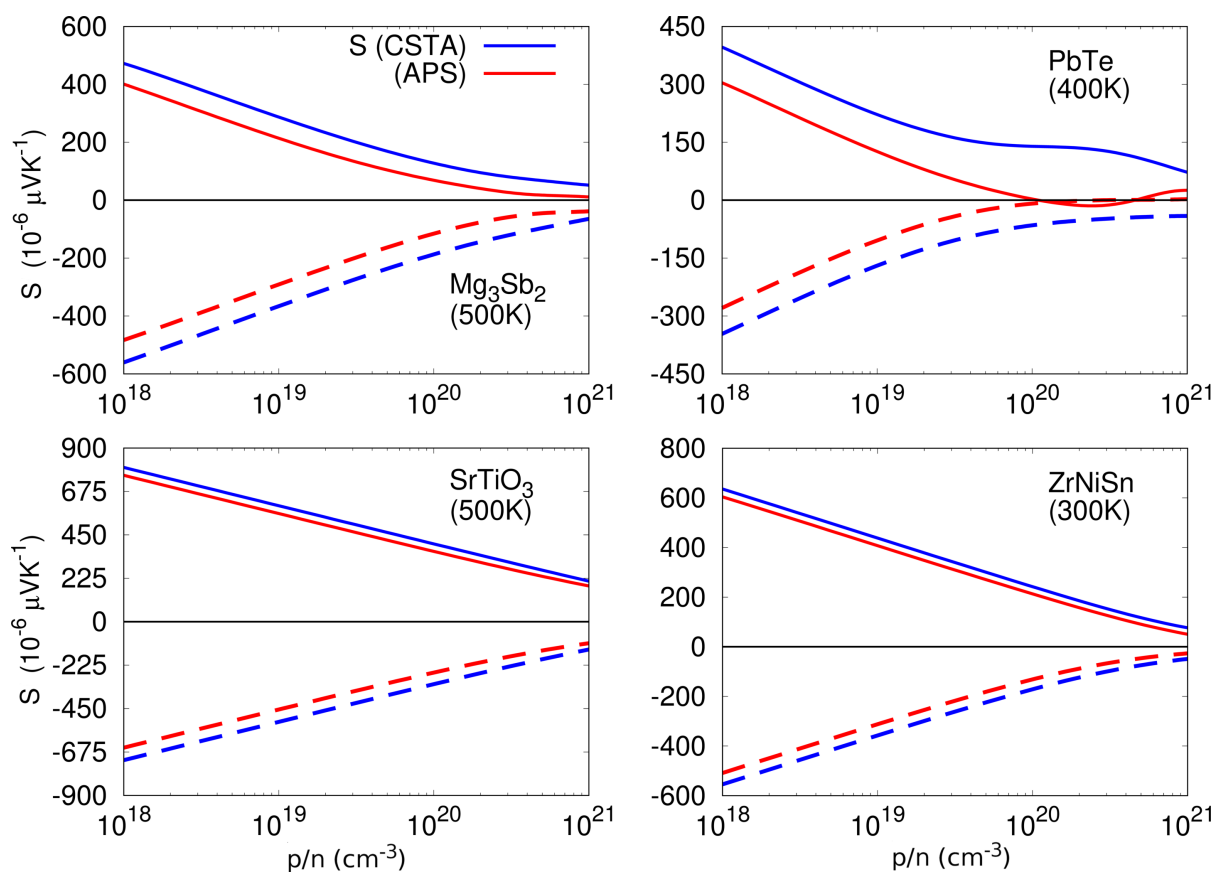

Figure 4.1: Calculated $S$ (in $\mu \mathrm{VK}^{-1}$ ) under both CSTA (blue) and APS (red) approximation as a function of doping concentration $\left(\right.$ in $\mathrm{cm}^{-3}$ ) of either doping types for four TEs at suitable temperatures. The solid lines indicate $p$-type whereas $n$-type doping has been shown in dashed lines.

scattering approximations. The results are shown in figure 4.2. When compared with the degenerate case, one may observe how the $L$ obtained using the standard definition gives us orders of magnitude large deviations, especially around lower doping levels. One may also note how $L$ decreases monotonically for $\mathrm{ZrNiSn}$ and $\mathrm{SrTiO}_{3}$ so as to converge to the degenerate limit value at highest doping levels. $\mathrm{PbTe}$ and $\mathrm{Mg}_{3} \mathrm{Sb}_{2}$ exhibit a local minima where they go below $L_{0}$ in the APS case before converging similarly to $L_{0}$.

Including the open circuit correction term as in equation $4.7 \mathrm{~b}$, we obtained the relevant estimate of $L$. This leads us to make a direct comparison of our first-principles 

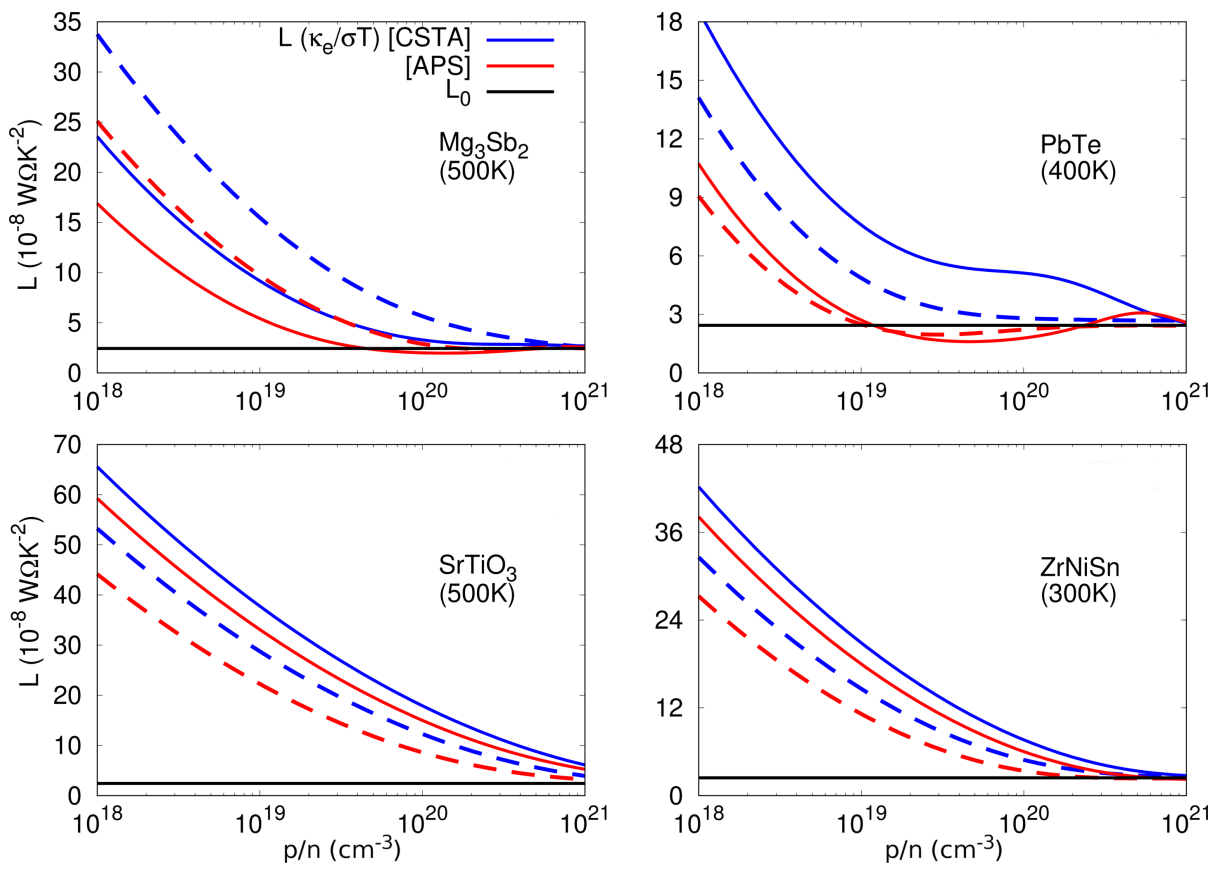

Figure 4.2: Calculated $L$ (in $\mathrm{W} \Omega \mathrm{K}^{-2}$ ) using equation 4.7a under both CSTA and APS approx. similar to figure 4.1 for both doping types.

results to the estimated $L$ in equation 4.1 . The results have been plotted against the doping concentration separately for $p$ and $n$ types in figure 4.3 and figure 4.4 , respectively. It should be noted that due to bipolar reduction of $S$, the $L$ vs $S$ relation is multivalued in certain materials. This suggests extra care when using expression 4.1, especially for narrow band gap materials which show strong bipolar transport [134]. The standard parabolic band results are also included in the fourth panel for comparison.

From figure 4.3, one may observe that the estimate of equation 4.1 gives reasonable agreement for $\mathrm{SrTiO}_{3}$ and $\mathrm{ZrNiSn}$. The results are summarized in table 4.2. For either of the compounds, we found the estimates to have a maximum deviation of less than about 15\%. Both of these have high doping levels. For the other two com- 

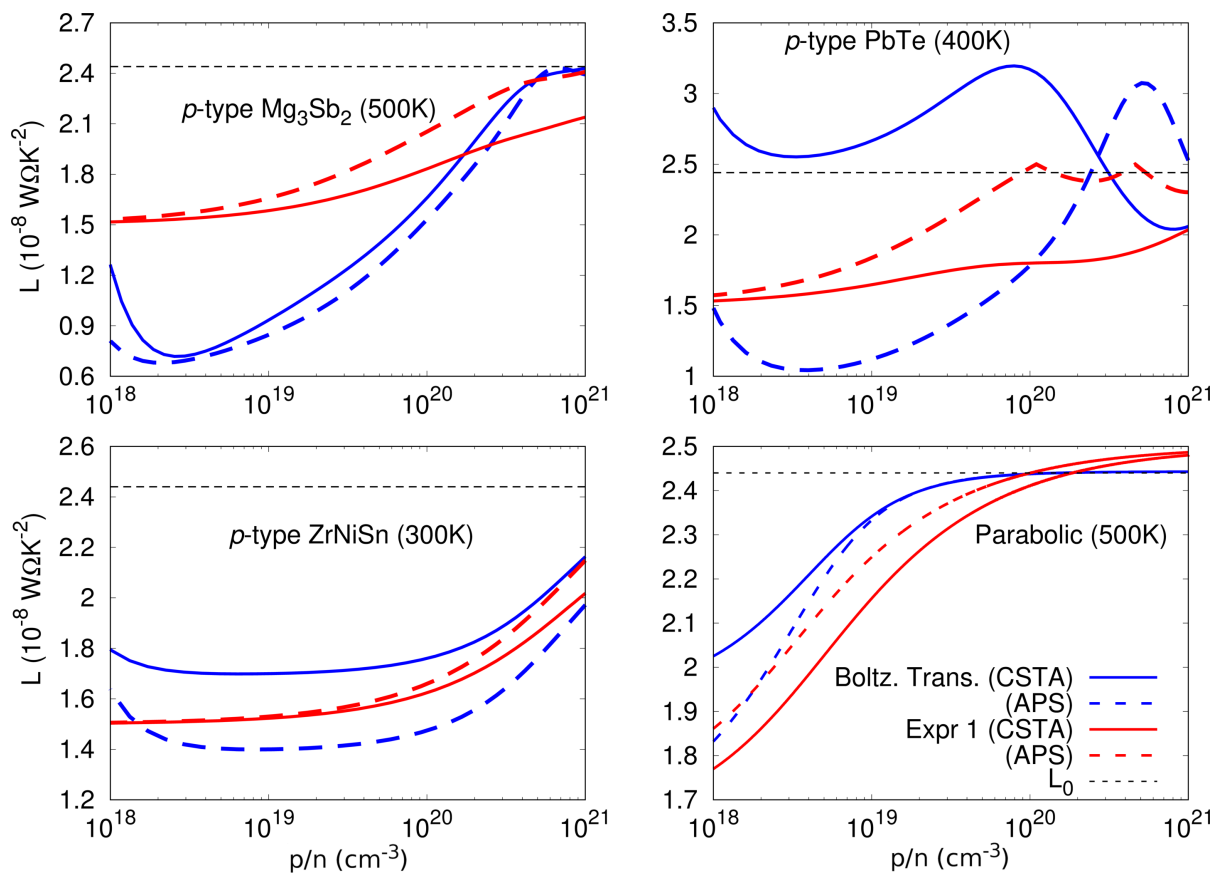

Figure 4.3: First-principles results of $L$ (in $\mathrm{W} \Omega \mathrm{K}^{-2}$ ) vs $p$-doping concentration using equation 4.7b under both CSTA (solid lines) and APS (dashed lines) shown in blue lines for above mentioned TEs at suitable temperatures. The approximation using expression 4.1 have been shown in red similarly. Standard degenerate limit value has been also been shown in black dashed lines.

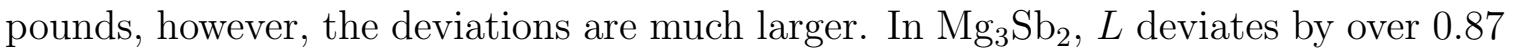
and by $0.35\left(\times 10^{-8} \mathrm{~W} \Omega \mathrm{K}^{-2}\right)$ for the $p$ and $n$-type, respectively. Similarly, for PbTe, these values are about $52 \%$ and $36 \%$. Interestingly, for the latter two compounds, these points lie closer to the non-degenerate doping limit.

The above mentioned deviations from $L_{0}$ are in accord with the results reported by Kim et al. [117] with a common overestimation trend among all four materials. However, it must be noted that especially for $\mathrm{Mg}_{3} \mathrm{Sb}_{2}$ (and also for $\mathrm{PbTe}$ somewhat), the deviations are quite large as the calculated $L$ dips much lower than the non-degenerate limit of $2\left(k_{B} / e\right)=1.5 \times 10^{-8} \mathrm{~W} \Omega \mathrm{K}^{-2}$ for the APS case. This is in accord with an 

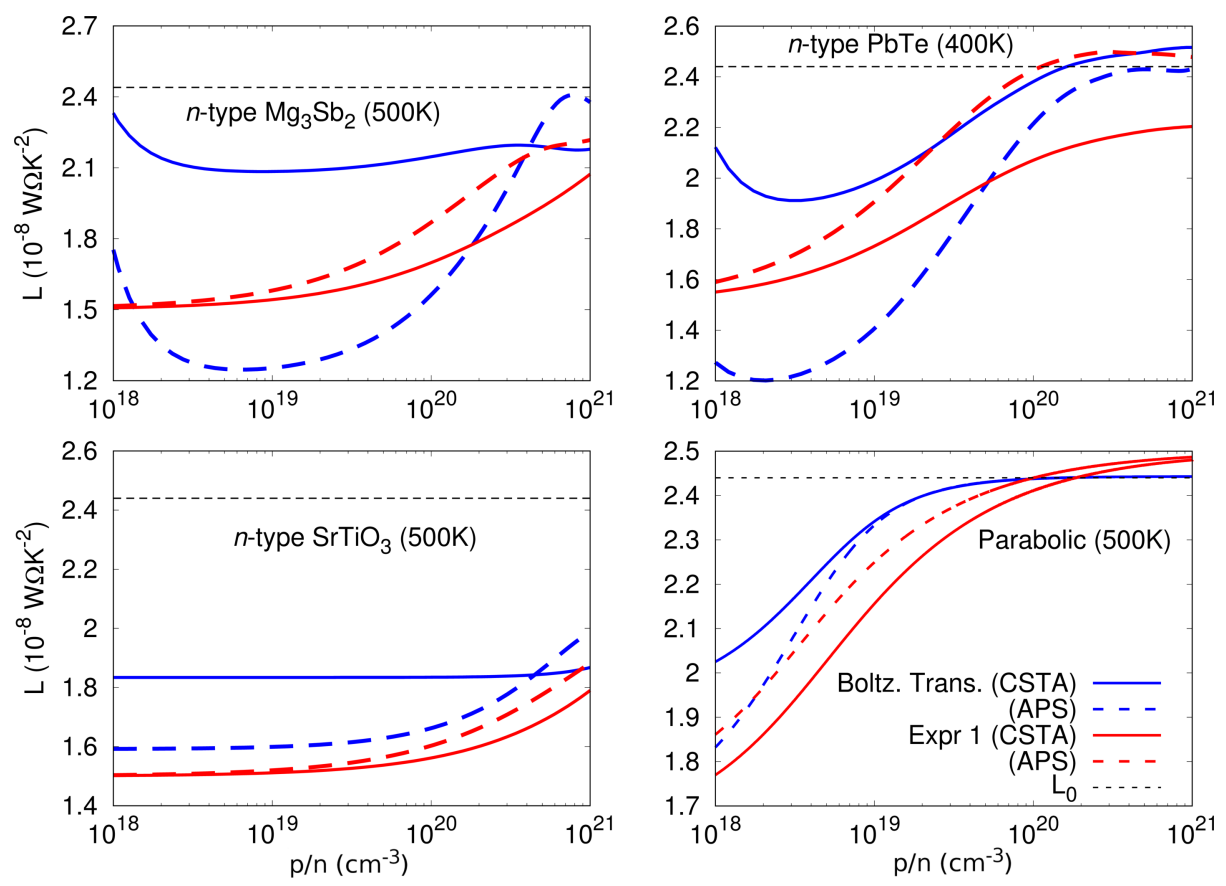

Figure 4.4: Calculated $L$ (in $\mathrm{W} \Omega \mathrm{K}^{-2}$ ) using equation 4.7b under both CSTA and APS time approximations similar to figure 4.3 for $n$-doped TEs at suitable temperatures.

Table 4.2: Maximum deviation between the estimated and calculated $L(L(|S|)-$ $\left.L\left(k_{\text {open }}\right)\right)$ with relevant doping concentration (conc.) in multiples of $10^{19} \mathrm{~cm}^{-3}$.

\begin{tabular}{ccccccc}
\hline \hline & \multicolumn{3}{c}{ Maximum deviation (in $10^{-8}{\mathrm{~W} \Omega K^{2}}^{2}$} \\
\cline { 2 - 7 } Material & \multicolumn{3}{c}{$p$} & \multicolumn{4}{c}{$n$} \\
& Conc. & Abs & $\%$ & Conc. & Abs & $\%$ \\
\hline $\mathrm{Mg}_{3} \mathrm{Sb}_{2}$ & 0.2 & 0.87 & 130 & 4.5 & 0.35 & 26 \\
$\mathrm{PbTe}$ & 4.5 & 0.76 & 52 & 0.9 & 0.5 & 36 \\
$\mathrm{SrTiO}_{3}$ & 0.13 & -0.21 & 12.3 & 150 & 0.2 & 13 \\
$\mathrm{ZrNiSn}$ & 20 & 0.2 & 13 & 5.0 & 0.17 & 11 \\
\hline \hline
\end{tabular}

earlier report where it was observed in the case of $\mathrm{Mg}_{3} \mathrm{Sb}_{2}$ that the low $L$ is beneficial for TE performance [126]. On the other hand, for PbTe, our results were in accord with those of a previous study, in which Ahmad and Mahanti [125] solved Boltzmann transport equations for Kane bands under different scattering mechanisms. 
Importantly, in addition to this, for the SPB results, we found that the approximation (simplified form) overshoots the degenerate value at extreme doping concentration, in agreement with the low $\mathrm{S}$ region as reported [117]. It also shows considerable deviations around the doping region of $10^{19} \mathrm{~cm}^{-3}$, the one relevant in TEs. The inclusion of our CSTA results shows the similarity of approximated $L$ across both scattering cases, especially at lower doping levels where $S$ is large. As reported, our first-principles results in figure 4.3, figure 4.4 confirm how this approximation is valid only for the APS case.

From equation 4.2, one may note how the transport coefficients involve integration of energy moments of the transport function $\Xi_{\mathrm{CSTA}}(E)$ over the electronic states chosen by the temperature-dependent window function $f^{\prime}\left(E-E_{F}\right)$. We plotted these quantities along with the characteristic DOS of the material in figure 4.5.

From figure 4.5, one may note how the widths of each window differ for each order of the moment of energy over the scale of a few $k_{B} T$. Thus, they sample the transport function $\Xi(E)$ differently. These different samplings control the different transport coefficients, and also the $L$. The DOS plots also show considerable deviations from their ideal parabolic nature.

It is evident from the above mentioned results, in particular the non-trivial doping dependence of $L$ in the top panels of figure 4.3, figure 4.4, that the proposed formula works within acceptable error ranges for $\mathrm{SrTiO}_{3}$ and $\mathrm{ZrNiSn}$. However, there are very 

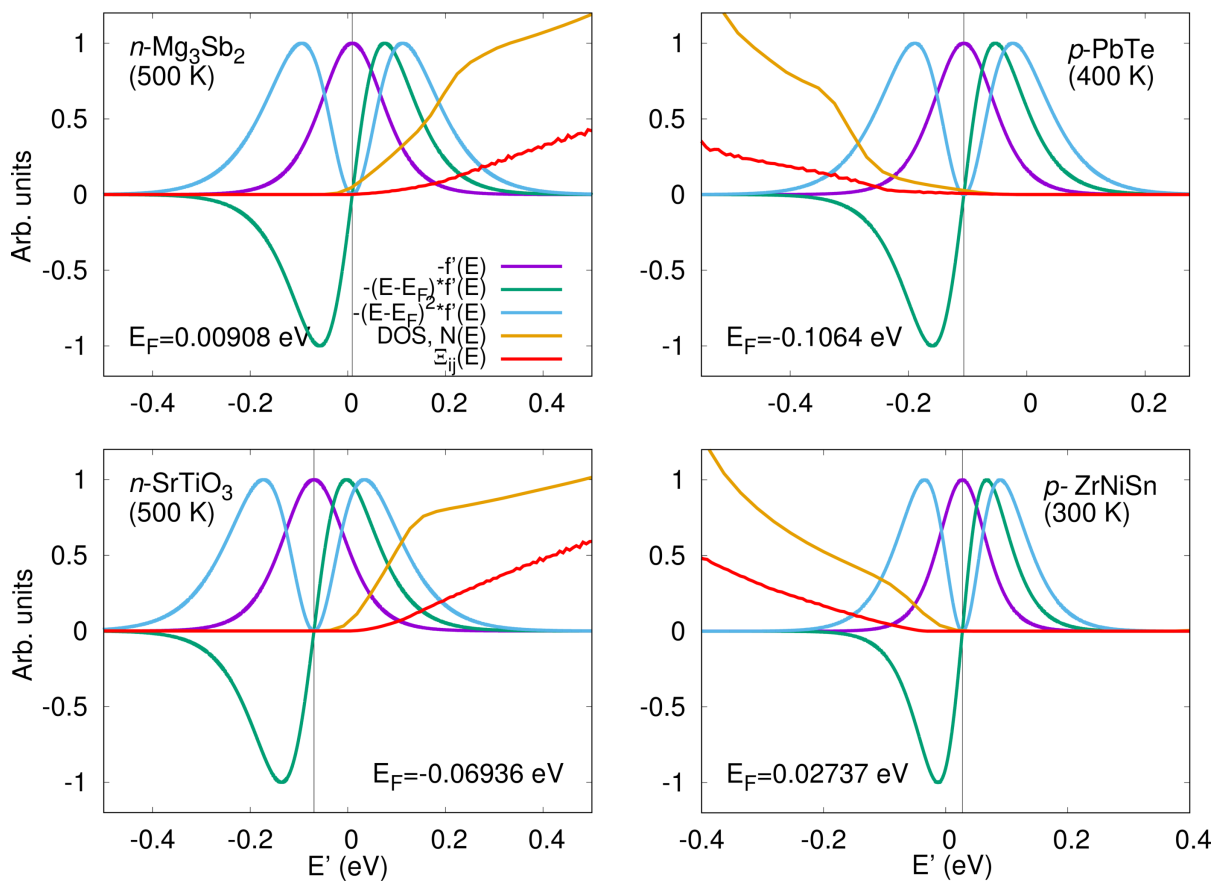

Figure 4.5: The window function: $f^{\prime}\left(E-E_{F}\right)$ plotted vs energy together with its various energy moments all scaled to unity. The doping types have been chosen for the one with better TE performance from literature at a fixed concentration of $5 \times 10^{19} \mathrm{~cm}^{-3}$. The integrand: transport function in equation 4.2 for the CSTA case along with the electronic DOS have also been shown for reference while scaling them appropriately for visibility. A smoothening of 0.003 Ry has been applied for all DOS plots. The zero energy was set at the nearest band edge.

sizable errors in determining the properties of compounds such as $\mathrm{Mg}_{3} \mathrm{Sb}_{2}$ and $\mathrm{PbTe}$. This is a consequence of the complex non-parabolic band structures, which are also of importance for their TE performance [92, 87, 135, 136]. Hence, care must be taken for materials having such non-parabolic band structures. From equations 4.2 and 4.7, it can be seen how the dependence of $L$ on $\Xi_{\mathrm{ij}}(\mathrm{E})$ is a much complex relationship. Thus, relating it directly to $|S|$ is an oversimplification. In case of these complex band structured TE materials, relying on this expression for $\kappa_{e}$ and $\kappa_{l}$ separation that yields an erroneous $L$ with an upper bound of $20 \%$ deviation as found above, 
might not be the right choice[120, 137].

\subsection{Conclusions}

We studied the accuracy of the expression proposed to estimate $L$, the Lorenz number, using the experimental $S$ by testing them against first-principles results obtained by solving Boltzmann transport equation using first-principles electronic structures as input. The equation is straightforward and is an improvement over using only the degenerate limit value: $L_{0}$ and works within $<15 \%$ error for TEs like $\mathrm{SrTiO}_{3}$ and $\mathrm{ZrNiSn}$. As a result this is expression is widely accepted choice for the experimental study of TEs that involves separation of the constituting electronic and lattice components of the thermal conductivity. However, the errors are large for materials known to have non-parabolic band structures and/or low-band gaps. As proposed originally, the formula also deviates around 5\% from the analytical SPB model in the doping concentration of around $10^{19} \mathrm{~cm}^{-3}$, that is, the range relevant to many well-known TEs. These findings are in agreement with previous results by Wang et al. [123] on $\mathrm{Si}$ and $\mathrm{Bi}_{2} \mathrm{Te}_{3}$. As mentioned, complex non-parabolic band structures are generally helpful in improving TE performance. Interestingly, we also find that materials such as $\mathrm{PbTe}$ and $\mathrm{Mg}_{3} \mathrm{Sb}_{2}$ for which this is the case are cases where the proposed expression also has larger errors. Therefore, although the expression remains useful, caution should be used in its application to TE materials with complex, non-parabolic band structures. 


\section{Chapter 5}

\section{Magnetism: a case study of its localized type}

This chapter and the following two, focuses on one of the major characteristics of condensed matter systems: magnetism, the origin and types of which are often decided by its intricate microscopic properties. Studying magnetism not only drives novel technological innovations, but also serves as an important tool to understand the detailed nature of physical phenomena operating at the atomic level of matter [138].

Here, in this chapter, we will find how a material's crystal structure, chemical composition and nature of electronic interactions leads it towards magnetism. I studied manganese selenite, a perovskite material of rare structural characteristics, and compare it with its non-magnetic counterpart zinc selenite in order to decipher the nature of its magnetism. Upon investigation of its properties, I find that this material 
is of localized magnetic nature. In the following two chapters, we will investigate the other kind of magnetism, viz:: the itinerant magnetism and some interesting phenomena associated with it.

\subsection{Properties of the antiferromagnetic selenite $\mathrm{MnSeO}_{3}$ and its non-magnetic analogue $\mathrm{ZnSeO}_{3}$}

I present our study of properties of antiferromagnetic selenite $\mathrm{MnSeO}_{3}$ and the nonmagnetic analogue $\mathrm{ZnSeO}_{3}$, based on first principles calculations. These compounds are rare examples of $\mathrm{ABO}_{3}$ perovskites with a tetravalent A-site and a divalent Bsite. The electronic structure is discussed in the context of the bonding and crystal structure. There is cross-gap hybridization between the $\mathrm{O} p$ states that form the valence bands of these compounds and the unoccupied $p$ states of Se, reflecting the lone pair physics that leads to the strong off-centering of Se from the perovskite A-site. The G-type antiferromagnetism of $\mathrm{MnSeO}_{3}$ is a local moment in nature arising from high spin $\mathrm{Mn}^{2+}$ with short range interactions. Additionally, there is an interesting spin-dependent hybridization of $\mathrm{Mn} d$ and $\mathrm{O} p$ states analogous to that in colossal magnetoresistance manganites. 


\subsection{Introduction}

Perovskite oxides constitute an exceptionally broad class of compounds, which exhibit many diverse properties and useful functionalities. The structure type is characterized by an $\mathrm{ABO}_{3}$ stoichiometry, based on corner linked $\mathrm{BO}_{6}$ octahedra arranged on a simple cubic lattice, often distorted, but always having this topology. This includes the four member rings that allow coherent rigid body rotations of the octahedra and that allow accommodation of ions with sizes mismatched to the ideal structure.

$\mathrm{MnSeO}_{3}$ is an antiferromagnetic compound, Néel temperature $T_{\mathrm{N}}=53.5 \mathrm{~K}$ [139], that occurs in an orthorhombic (spacegroup 62, Pnma) structure that has been described as a distorted perovskite and as an ionic salt consisting of $\mathrm{Mn}^{2+}$ and $(\mathrm{SeO} 3)^{2-}$ [140]. It belongs to a group of selenites described by the chemical formula $\mathrm{MSeO}_{3}$, where $\mathrm{M}$ is a metal $(\mathrm{Mn}, \mathrm{Co}, \mathrm{Ni}, \mathrm{Zn}, \mathrm{Mg}$ or $\mathrm{Cu}$ ). These compounds are generally formed at elevated pressure and temperature [139, 140, 141, 142, 143]. First principles calculations for several of these selenites, including $\mathrm{MnSeO}_{3}$ were reported by Michel [143].

Common A-B valence states for $\mathrm{ABO}_{3}$ perovskites are 0-6 (e.g. $\mathrm{WO}_{3}$ ), 1-5 (e.g. $\mathrm{KTaO}_{3}$ ), 2-4 (e.g. $\mathrm{PbTiO}_{3}$ ) and 3-3 (e.g. $\mathrm{BiFeO}_{3}$ ) [144]. However, these selenites are examples of the rarely seen 4-2 valence combination (4 on the A-site and 2 on the B-site). The reason for this rarity is well understood in terms of the Pauling rules where the corner sharing perovskite structure is normally stabilized by repulsion between highly charged B-site cations [145], which in the present compounds are not

highly charged. Furthermore, the perovskite tolerance factors $\left(t=0.63\right.$ for $\left.\mathrm{ZnSeO}_{3}\right)$, 
based on Shannon crystal ionic radii, are extremely low for a stable perovskite due to the small size of the $\mathrm{Se}^{4+}$ cation. The structure of these selenites accommodates the low tolerance factor via a strong off-centering of the Se ions in their cage and tilts of the $\mathrm{BO}_{6}$ octahedra, as is common in low tolerance factor perovskites. It has been suggested that these tilts play an important role in the exchange interactions $[139,140,141,142,143]$. It is also of interest to note that $\mathrm{MnSeO}_{3}$ can be regarded as a charge conjugate analogue of $\mathrm{SrMnO}_{3}$, in the sense that with high spin $\mathrm{Mn}$ in an octahedral crystal field, doping the full $\mathrm{e}_{g}$ level of $\mathrm{MnSeO}_{3}$ with holes is like doping $\mathrm{SrMnO}_{3}$ with electrons, as in the ( $\left.\mathrm{La}, \mathrm{Sr}\right) \mathrm{MnO}_{3}$ colossal magnetoresistance (CMR) system $[146,147,148]$.

Here I report a first principles investigation of $\mathrm{MnSeO}_{3}$ and the non-magnetic analogue $\mathrm{ZnSeO}_{3}$ to elucidate the nature of the exchange interactions and the basic features of the electronic structure in relation to the bonding and crystal structure.

\subsection{Computational approach}

The calculations presented here were done in the framework of density functional theory using the general potential linearized augmented planewave (LAPW) method [6] as implemented in the WIEN2k code [5]. We used the generalized gradient approximation (GGA) of Perdew, Burke and Ernzerhof (PBE) [17], along with well converged Brillouin zone samplings and basis sets. We used LAPW sphere radii of 1.4 Bohr, 1.8 Bohr, 2.0 Bohr and 2.0 Bohr, for O, Se, Mn and Zn, respectively, and 
a planewave cutoff, $\mathrm{K}_{\max }$, determined by $R_{\min } K_{\max }=7.0$, where $R_{\min }=1.4$ Bohr is the $\mathrm{O}$ sphere radius. This yields effective $R_{\mathrm{mt}} K_{\max }$ values of at least 9.0 for the other atoms. Local orbitals were used to include the semicore states with the valence states. We tested different zone sampling densities for convergence. The results shown were obtained with meshes of $10 \times 8 \times 12$ or better. We also calculated magnetic energies using the PBE $+\mathrm{U}$ method, with $U=5 \mathrm{eV}$ to test the effect of possible beyond density functional theory correlation effects. These calculations were done with fully localized limit double counting as implemented in the WIEN2k code.

Both $\mathrm{ZnSeO}_{3}$ and $\mathrm{MnSeO}_{3}$ occur with orthorhombic Pnma structures, with four formula units per cell. We used experimental lattice parameters, and relaxed the atomic positions using the PBE GGA. Magnetism was included for $\mathrm{MnSeO}_{3}$. The crystal structures are depicted in figure 5.1. For $\mathrm{ZnSeO}_{3}$, which is semiconducting, we also did calculations using the modified Becke Johnson (mBJ) potential [23]. As described in earlier chapters, compared to standard GGA functionals designed for total energies $[17,18]$, such as the PBE GGA, this potential generally gives much improved band gaps in relation to experiment for simple semiconductors and insulators, although it is not applicable to magnetic transition metal compounds, such as $\mathrm{MnSeO}_{3}[75,74]$. Optical properties for $\mathrm{ZnSeO}_{3}$ were obtained with the optical package of the WIEN2k code using the mBJ predicted electronic structure. 

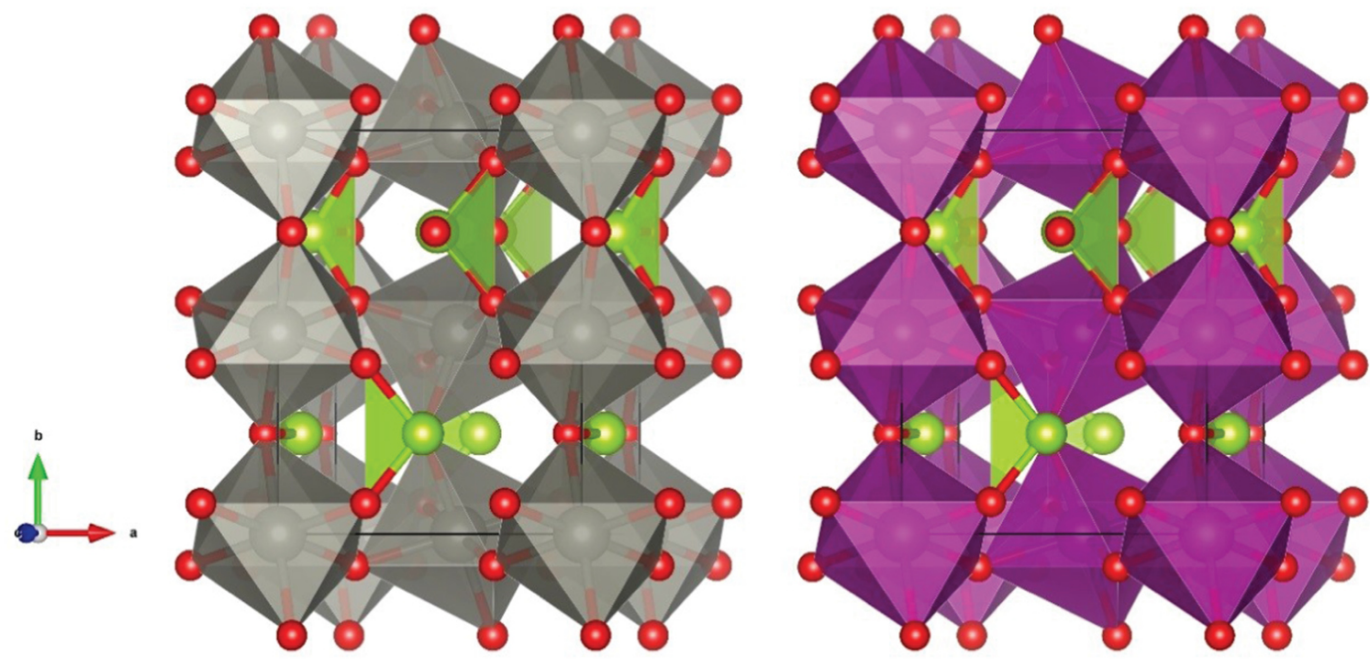

Figure 5.1: Structure of $\mathrm{ZnSeO}_{3}$ (left) and $\mathrm{MnSeO}_{3}$ (right). The $\mathrm{Zn} / \mathrm{Mn}$ are in the centers of the octahedra. Note the perovskite structural motif of corner sharing $\mathrm{ZnO}_{6}$ or $\mathrm{MnO}_{6}$ octahedra, although with very strong rotations.

\subsection{Results and discussion}

The structure (figure 5.1) consists of fully corner linked M (Zn or Mn) centered octahedra. Structural parameters are given in table 5.1. The structure is perovskite-like in terms of the connectivity and topology of the $\mathrm{MO}_{6}$ octahedra. However, it is strongly distorted as seen from the very strong deviation of the $\mathrm{M}-\mathrm{O}-\mathrm{M}$ bond angles from the ideal value of $180^{\circ}$. The angles are in fact substantially smaller than even in the proton conductor, $\mathrm{CaZrO}_{3}\left(\sim 145^{\circ}\right.$ [149]). On the other hand, the octahedra themselves are relatively undistorted in both compounds. The similar, small level of distortion for the Zn and Mn compounds implies an absence of Jahn-Teller activity in the $\mathrm{Mn}$ compound, consistent with its nominal $\mathrm{Mn}^{2+}$ valence. More generally, the structural similarity of the two compounds strongly supports this assignment of $\mathrm{Mn}^{2+}$ . However, in both compounds the $\mathrm{M}$ bond valence sum is somewhat higher than the nominal value (2.30 for $\mathrm{ZnSeO}_{3}$ and 2.24 for $\mathrm{MnSeO}_{3}$ ), implying Se-O covalency. 
Table 5.1: Structural parameters for Pnma $\mathrm{ZnSeO}_{3}$ and $\mathrm{MnSeO}_{3}$. The lattice parameters, $a, b$ and $c$ are from experiment $[139,140,141,142,143]$. Each $M=\mathrm{Zn}, \mathrm{Mn}$ has six $\mathrm{O}$ neighbors, two at each independent bond length. Se has three short bond lengths, with other Se-O distances longer than $2.8 \AA$. There are two independent $M-\mathrm{O}-M$ bond angles, one connecting octahedra along the $b$ direction (denoted (b)), and two equal angles connecting in the ac plane.

\begin{tabular}{cll}
\hline \hline & \multicolumn{1}{c}{$\mathrm{ZnSeO}_{3}$} & \multicolumn{1}{c}{$\mathrm{MnSeO}_{3}$} \\
\hline$a, b, c(\AA)$ & $5.9239,7.6684,5.0421$ & $6.093,7.8638,5.1426$ \\
$M-\mathrm{O}(\AA)$ & $2.075,2.168,2.208$ & $2.153,2.234,2.267$ \\
$\mathrm{Se}-\mathrm{O}(\AA)$ & $1.740,1.754,1.754$ & $1.743,1.755,1.755$ \\
$M-\mathrm{O}-M$ & $124.3^{\circ}(\mathrm{b}), 130.5^{\circ}$ & $123.3^{\circ}(\mathrm{b}), 128.8^{\circ}$ \\
\hline \hline
\end{tabular}

The strong distortion from the ideal perovskite structure could perhaps be rationalized by the very small size of the $\mathrm{Se}^{4+}$ ion, which would in turn lead to a very low tolerance factor of $\sim 0.6$ in these compounds. However, this is outside the normal range of perovskite stability [150]. In fact, as seen, the Se is strongly off-centered within its anion cage, leading to three very short Se-O bonds $(\sim 1.75 \AA)$, while the other Se-O distances are all longer than $2.8 \AA$. The short bond lengths are again suggestive of at least some significant degree of Se-O covalency. From this point of view, the stability of these compounds with perovskite topology is due to the bonding of Se with three O neighbors. This can also be discussed as a strong lone pair driven distortion (note that lone pair distortions implicitly involve cation ligand $p$-electron hybridization).

The electronic density of states (DOS) for $\mathrm{ZnSeO}_{3}$ is shown in figure 5.2. Se $p$ and O $p$ projections of the DOS are shown in figure 5.3, and the band structure is given in figure 5.4. The present calculations were done using small Se and O LAPW sphere 
radii of 1.8 Bohr and 1.4 Bohr. This was necessitated by the short Se-O bond lengths. Note that as a result of this and the extended nature of Se $p$ and $\mathrm{O} p$ orbitals, these projections are approximately proportional to, but lower than the $p$ orbital character. In any case, the plot clearly shows covalency involving the nominally Se $p$ derived conduction band orbitals and the nominally $\mathrm{O} p$ derived valence band orbitals through the sizable Se character in the valence bands and O character in the conduction bands.

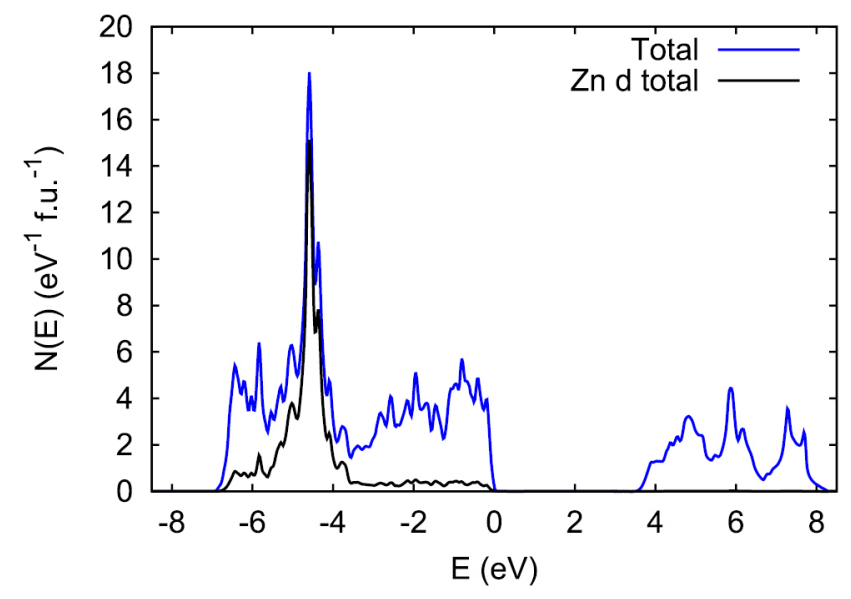

Figure 5.2: Electronic density of states and $d$-character projection onto the Zn LAPW sphere for $\mathrm{ZnSeO}_{3}$, as obtained with the PBE GGA on a per formula unit basis.

Finally, before turning to the electronic structure and magnetism, we note that the strongly bent Mn-O-Mn bond angles may be expected to have a strong influence on the magnetic interactions. These angles are in the range where antiferromagnetic superexchange may be weakened in favor of ferromagnetic interactions according to the Goodenough-Kanamori rules $[151,152]$. The distortion is stronger for the connections along the $b$ axis direction, which may suggest perhaps different interactions along $b$. 


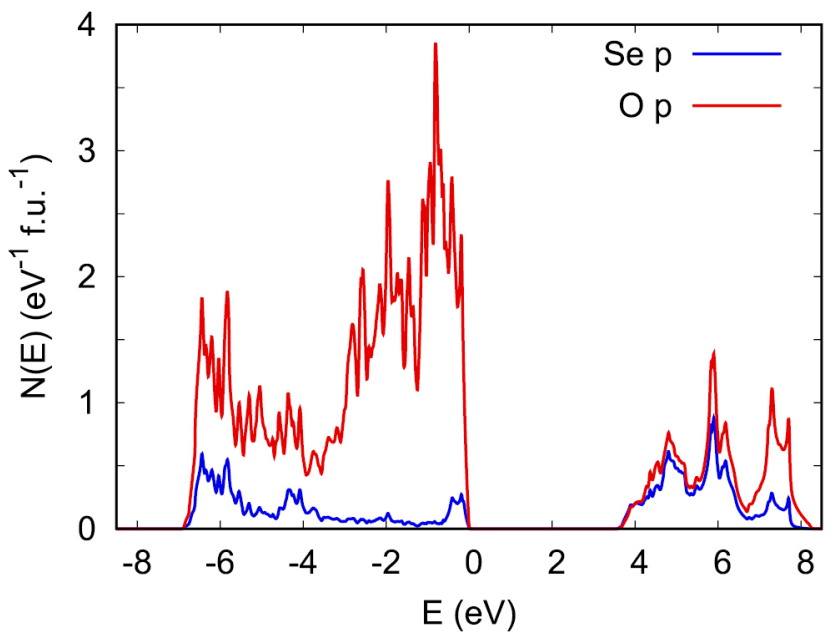

Figure 5.3: Projection of the DOS of $p$ character onto the Se and O LAPW spheres in $\mathrm{ZnSeO}_{3}$ as obtained with the PBE GGA. Note that due to the small Se and O LAPW sphere radii this is approximately proportional to but smaller than the true $p$ orbital characters.

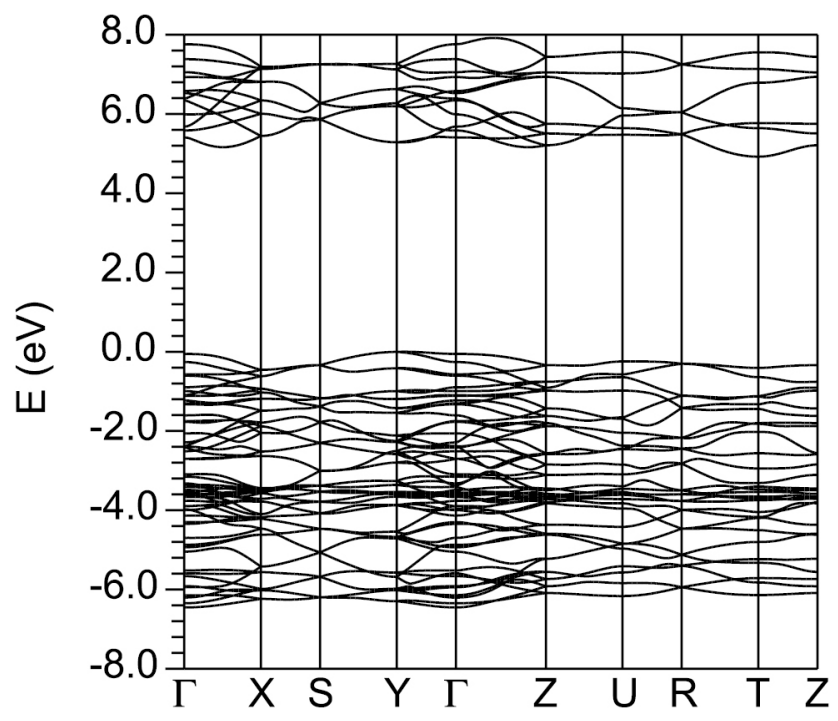

Figure 5.4: Calculated band structure of $\mathrm{ZnSeO}_{3}$ as obtained with the mBJ potential.

We begin the discussion of the electronic structures with $\mathrm{ZnSeO}_{3}$, which can be regarded as a non-magnetic reference for $\mathrm{MnSeO}_{3}$. figure 5.2 shows the calculated electronic DOS as obtained with the PBE GGA. As seen, the compound is insulating 
and the $\mathrm{Zn} d$ states are fully occupied and occur at a binding energy of $\sim 5 \mathrm{eV}$ relative to the valence band maximum, consistent with the expected divalent Zn. The width of the $\mathrm{O} p$ derived valence bands is $\sim 6.4 \mathrm{eV}$ and the almost certainly underestimated PBE band gap is $3.8 \mathrm{eV}$.

Calculations with the mBJ potential give a larger band gap of $\sim 4.9 \mathrm{eV}$. The optical absorption spectrum is given in figure 5.5. As seen, there is an onset of strong absorption starting slightly above the fundamental band gap, with anisotropy near the band edge.

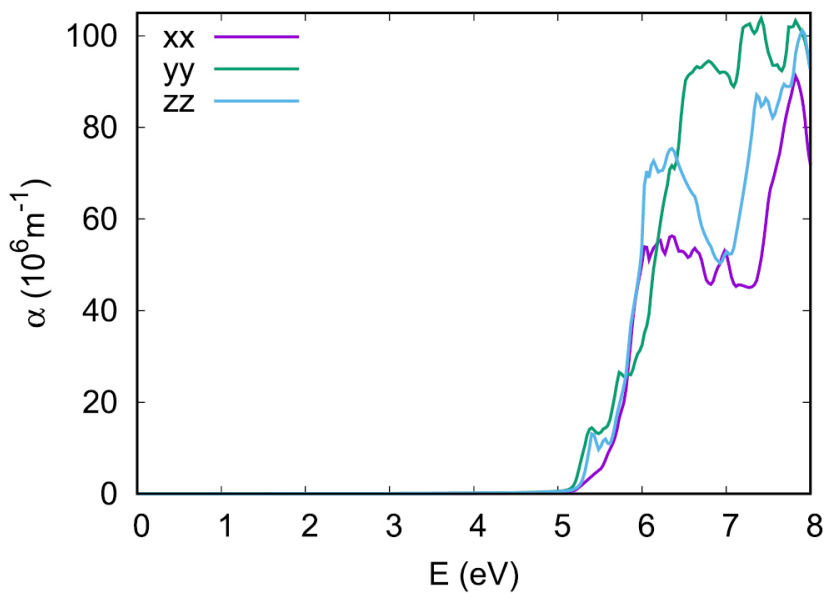

Figure 5.5: Calculated optical absorption spectrum for $\mathrm{ZnSeO}_{3}$ as obtained with the mBJ potential.

We performed calculations for $\mathrm{MnSeO}_{3}$ with different assumed magnetic orders. In all cases, we obtained an insulating electronic structures and moments consistent with high spin $\mathrm{Mn}^{2+}$. Specifically, we obtained fully occupied Mn majority $d$ bands and unoccupied minority $d$ bands. The orders that we considered were ferromagnetic (F), G-type antiferromagnetic (G), with all nearest neighbor Mn-Mn connections 
antiferromagnetic, C-type (C), consisting of ferromagnetic chains along the $b$-axis direction, ordered with nearest neighbor antiferromagnetism in the ac plane and A-type (A), with ferromagnetic layers in the $a c$ plane stacked antiferromagnetically along $b$. The electronic structures are shown in figure 5.6. The band structure in the ground state G-type ordering is shown in figure 5.7. The basic features of the electronic structure of $\mathrm{MnSeO}_{3}$ are similar to those of $\mathrm{ZnSeO}_{3}$, e.g. in the $\mathrm{O} p$ derived valence band width, with the exception of the position of the $\mathrm{M} d$ bands. In $\mathrm{ZnSeO}_{3}$ these are fully occupied and overlap the $\mathrm{O} p$ bands, while in the case of $\mathrm{MnSeO}_{3}$ these are at the top of and above the $\mathrm{O} p$ bands.

We also performed calculations without spin polarization. We find that G-type antiferromagnetic order provides the lowest energy, which is consistent with experiment [139], and a prior calculation by Michel [143]. The calculated energies for the different structures are given in table 5.2.

Table 5.2: Energies of $\mathrm{MnSeO}_{3}$ per formula unit for different magnetic states (refer text). The energy for the lowest energy G-type order was taken as the zero. NSP denotes non-spin-polarized.

\begin{tabular}{cccccc}
\hline \hline Order & G & C & A & F & NSP \\
\hline $\mathrm{E}(\mathrm{eV})$ & 0.0000 & 0.0175 & 0.0325 & 0.0550 & 2.59 \\
\hline \hline
\end{tabular}

As seen, all magnetically ordered structures are much lower in energy than the non-spin polarized (NSP) case, and the energy differences between these orders is very much lower in magnitude than the energy difference from the NSP case (at most $0.055 \mathrm{eV}$ versus $2.59 \mathrm{eV}$ ). This means that the inter-site exchange interaction energies 


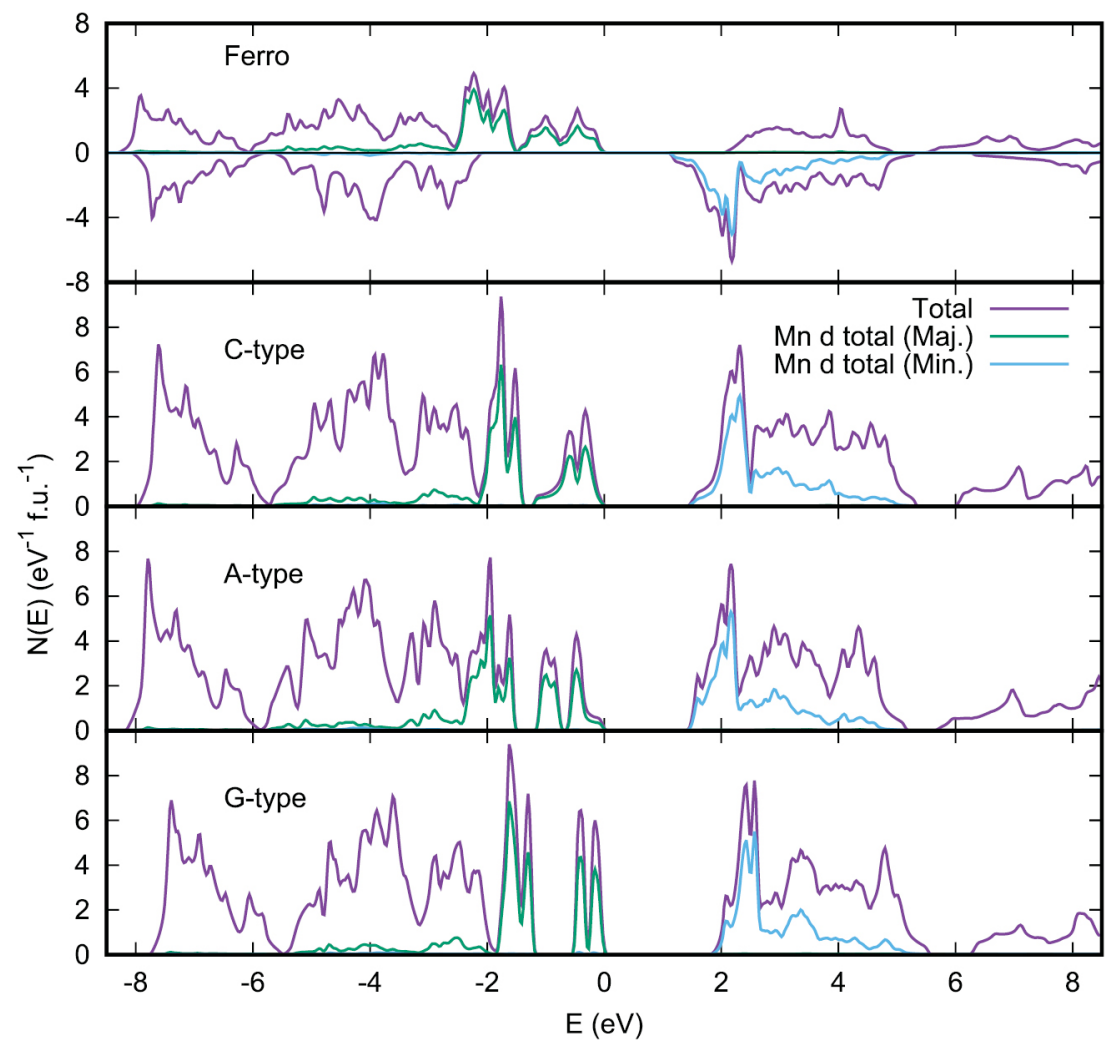

Figure 5.6: Calculated electronic density of states and minority and majority spin Mn $d$ projections for $\mathrm{MnSeO}_{3}$ with different magnetic orders. In the ferromagnetic case, majority spin is shown above the axis and minority spin is shown below.

are much weaker than the on-site energy associated with moment formation. $\mathrm{MnSeO}_{3}$ is therefore in the limit of a local moment antiferrromagnet. This is in contrast to e.g. $\mathrm{SrTcO}_{3}$, where the two energy scales are comparable resulting in an extremely high $\mathrm{T}_{\mathrm{N}}$ [153]. In any case, with only nearest neighbor interactions considered, based on the energy difference between the $\mathrm{F}$ and $\mathrm{G}$ orders, one obtains an average energy cost for a ferromagnetic bond of $0.018 \mathrm{eV}$. The difference between A- and G-type orders gives an energy cost of $0.016 \mathrm{eV}$ for a ferromagnetic bond in the ac plane, while the energy difference between $\mathrm{C}$ - and G-type give a cost of $0.018 \mathrm{eV}$ for a $b$-axis ferromagnetic bond. The resulting average from the calculated G-, A- and C-type orders 

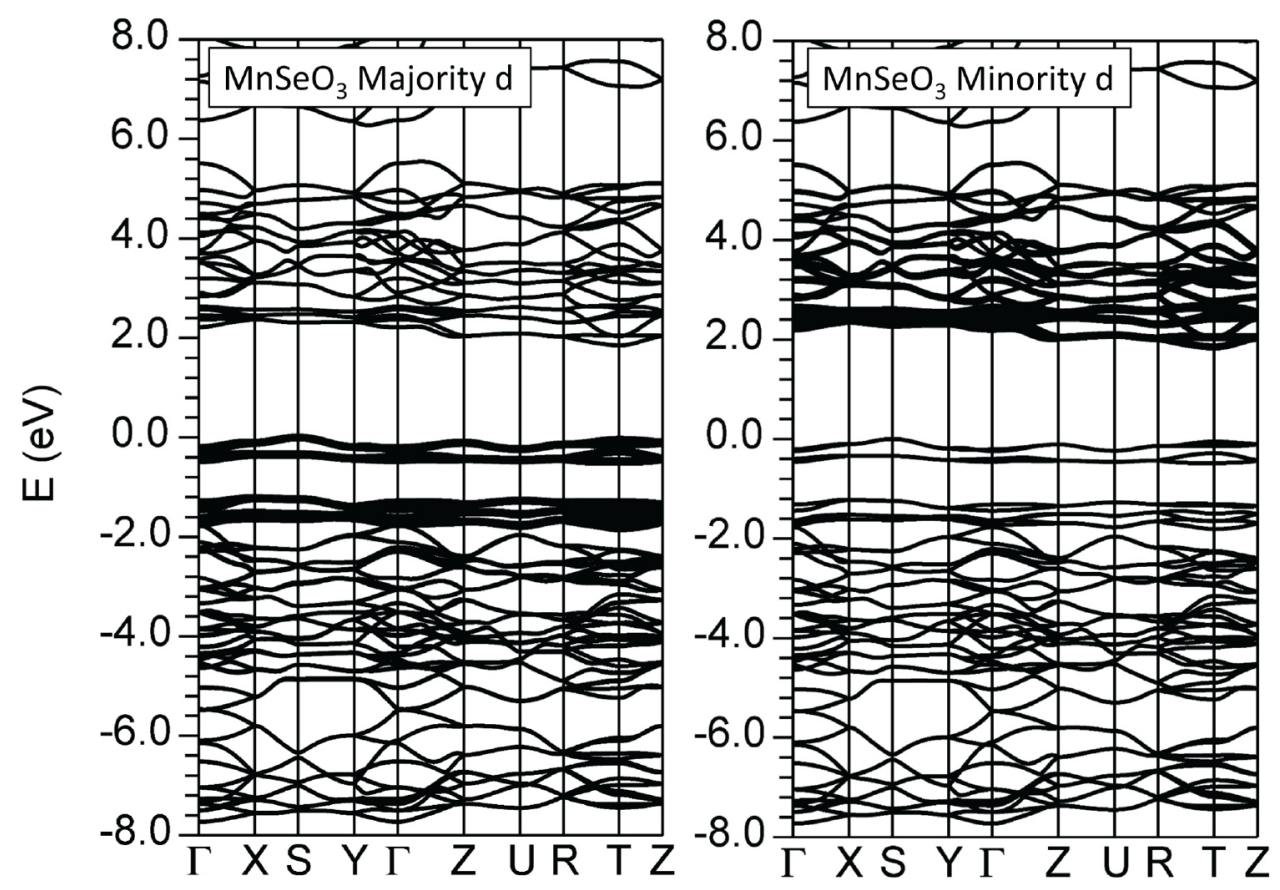

Figure 5.7: Calculated band structure of $\mathrm{MnSeO}_{3}$ with G-type antiferromagnetic order, emphasizing majority (left) and minority (right) Mn $d$ character using the 'fat-bands' scheme.

is then $0.017 \mathrm{eV}$, which is very close to that obtained using the $\mathrm{F}$ order calculation. Thus it seems that the magnetic energies may be well described in terms of a nearest neighbor interaction that is antiferromagnetic and only weakly anisotropic.

It is possible that correlation effects beyond the PBE level may be important in these compounds, although it should be noted that the high spin, half filled Mn d band implies a strong Hund's coupling that may work against additional effects of Coulomb repulsions. In any case, to determine the qualitative effects that additional Coulomb repulsion would have, we performed $\mathrm{PBE}+\mathrm{U}$ calculations with $\mathrm{U}$ set to $5 \mathrm{eV}$. These calculations were done using the fully localized limit double counting scheme. We find the same energy ordering as without $U$ and the same relative energy 
differences to within $10 \%$. The energy scale between the different magnetic orderings is, however, reduced. The energy difference between the G-type and ferromagnetic order becomes $0.015 \mathrm{eV} /$ formula unit, much smaller than the $0.055 \mathrm{eV}$ for the PBE GGA. This reflects the generally reduced hybridization in $\mathrm{PBE}+\mathrm{U}$ calculations relative to PBE calculations [154]. The qualitative trend is similar to that found by Michel with hybrid functionals [143]. Generally, the effect of $\mathrm{PBE}+\mathrm{U}$ is to raise the energy of the unoccupied transition metal $d$ bands relative to the occupied bands and $\mathrm{O} p$ bands. This increased energy separation reduces hybridization and therefore the strength of superexchange [155].

Turning to the electronic structure (figure 5.6), it may be noted that all magnetic orders show insulating gaps in excess of $1 \mathrm{eV}$. The calculated band gap for the lowest energy G-type order is $\sim 2 \mathrm{eV}$. The gap formation is due to the large exchange splitting on the high spin $\mathrm{Mn}^{2+}$ ions and is between occupied majority spin $\mathrm{e}_{g}$ bands and unoccupied $t_{2 g}$ bands. $\mathrm{MnSeO}_{3}$ is therefore an example of a transition metal oxide with an odd number of electrons per formula unit that is insulating at the GGA level independent of the specific magnetic order. Considering the very high energy scale for moment formation relative to the inter-site exchange interactions the paramagnetic state above $T_{N}$ should be viewed as having $\mathrm{Mn}$ ions with thermally fluctuating moment directions, but stable moments. Based on the GGA results, this would also be insulating even without considering electron correlations beyond the GGA level.

The DOS for the ferromagnetic order has $\mathrm{O} p$ bands that range from approximately $-2.5 \mathrm{eV}$ to $-9 \mathrm{eV}$, relative to the highest occupied state. There is pronounced hy- 
bridization between the $\mathrm{Mn} d$ and $\mathrm{O} p$ orbitals, and this hybridization is very spin dependent and much stronger in the majority spin channel. This is seen in the majority spin Mn $d$ projection, where there is a long tail extending into the $\mathrm{O} p$ bands. There is also evident hybridization, though weaker, between the unoccupied minority $\mathrm{Mn} d$ orbitals and the $\mathrm{O} p$ orbitals. This cross-gap hybridization provides an explanation for the antiferromagnetic exchange [155]. The exchange splitting of the Mn $d$ states, estimated from the ferromagnetic DOS, is $\sim 4.5 \mathrm{eV}$, while the $t_{2 g}-\mathrm{e}_{g}$ crystal field splitting is $\sim 1 \mathrm{eV}$. The large exchange splitting relative to the crystal field is consistent with the high spin Mn. The calculated spin moment for the ferromagnetic ordering was $5 \mu_{\mathrm{B}}$ per formula unit, of which $4.24 \mu_{\mathrm{B}}$ is contained inside the Mn LAPW sphere radius 2.0 Bohr, also consistent with high spin $\mathrm{Mn}^{2+}$. All the antiferromagnetic configurations also show spin dependent $\mathrm{Mn} d-\mathrm{O} p$ hybridization, similar to the ferromagnetic case. We note that strong spin dependent hybridization is also a characteristic of the CMR manganites [156, 157, 158, 159, 160].

\subsection{Summary and conclusions}

We report a first principles investigation of the nature of magnetism in the antiferromagnetic compound $\mathrm{MnSeO}_{3}$ and its non-magnetic analogue $\mathrm{ZnSeO}_{3}$. $\mathrm{ZnSeO}_{3}$ is predicted to be an insulator with a band gap near $4.9 \mathrm{eV}$ using the mBJ potential. We find that $\mathrm{MnSeO}_{3}$ is reasonably described as a perovskite oxide with a high spin $\mathrm{Mn}^{2+} \mathrm{B}$-site cation. We find a G-type lowest energy magnetic order in accord with experiment. The magnetism is in the local moment limit, with an insulating elec- 
tronic structure, The inter-site exchange coupling may be well described in terms of nearest neighbor nearly isotropic interactions. Additionally, the electronic structure shows strong spin dependent Mn $d-\mathrm{O} p$ hybridization. In light of this, in analogy with the CMR manganites, it will be of interest to determine whether the compound can be doped, and if so whether a conducting ferromagnetic state can be induced. In any case, inelastic scattering experiments to measure the spin-wave dispersions will be a useful for establishing the nature of the exchange interactions in relation to other manganites. 


\section{Chapter 6}

\section{Quantum criticality and the search for competing magnetic orders in $\mathrm{Sr}_{3} \mathrm{Ru}_{2} \mathrm{O}_{7}$}

The following two chapters focus on the material $\mathrm{Sr}_{3} \mathrm{Ru}_{2} \mathrm{O}_{7}$. This material is wellknown for showing a variety of interesting phenomenon like quantum criticality and electronic nematicity. In this chapter, starting with a brief exposition on quantum criticality, we discuss why it is essential to know about the low-energy states present in competition with the ground state of such a system and how such a competition directly influences its overall low-temperature ground state properties. This underlying competition in $\mathrm{Sr}_{3} \mathrm{Ru}_{2} \mathrm{O}_{7}$, when appropriately perturbed via different techniques, reveals many other properties and phases. Reviewing the literature to include them, we searched for such metastable states that consists of a specific magnetic order and also investigated its structural properties. 
A search strategy comprising of PAW pseudopotential based first-principles methods [25], we surveyed 12 possible magnetically ordered states. This finally yielded us with a striped magnetically ordered state that indeed lies energetically very close to the nascent density functional predicted ferromagnetic ground state. It is important to note here that in quantum critical systems comprised of a strongly fluctuating ground state, standard density functional methods almost always fail to directly capture its true ground state properties, often marked by a large overestimation of its magnetic moments etc. However, we discuss how this known issue is being dealt with and can be used advantageously in favor of these itinerant magnetic systems showing such signature properties [201] while still being able to reliably predict its metastable states.

This bilayered metal, however, being closely related to $\mathrm{Sr}_{2} \mathrm{RuO}_{4}$, which is a wellknown low temperature unconventional superconductor with elusive electronic interactions, shows another interesting property. In this direction, we continue our discussion of $\mathrm{Sr}_{3} \mathrm{Ru}_{2} \mathrm{O}_{7}$ where in the next chapter we focus on this phenomenon which is known as the electronic nematicity where under specific magnetic field, the in-plane transport properties of this metal displays a peculiar anisotropy. In this context, we performed transport calculations on all of various magnetically ordered states and found that such an anisotropy does exist exclusively in this metastable, striped magnetically ordered state. 


\subsection{Quantum critical point}

A critical point, as understood for a classical phase transition, which occurs at a finite temperature, is that point in the phase-space of a material where the line separating two phases in equilibrium, terminates. Such a phase transition is driven by thermal fluctuations of the system.

In contrast, a quantum phase transition is driven by quantum fluctuations, as identified by the Heisenberg's uncertainty principle. Also, such a phase transition takes place at absolute zero and can be accessed by tuning some non-thermal parameter of the system. At such a "quantum critical point" (QCP) the behavior of the material is characterized by [161] a state that is the quantum superposition of both order and disorder. Refer to figure 6.1. At this critical point, such quantum critical fluctuations (for a second order phase transition) exhibit scale invariance in both space and time i.e. the order parameter fluctuates over infinite region of space and time. As explained by Coleman and Schofield [162], as one approaches such a point, ever-larger "droplets" of "nascent" order develop and span the whole system.

One must note here that although interest in QCP arose purely for academic interests, recent experimental results led to the belief that the presence of such a QCP in a material can influence its electronic properties at non-zero temperatures, especially in high $\mathrm{T}_{\mathrm{c}}$ superconductors and a whole host of other superconductors that carry quasi-linear resistance signatures in their normal states $[162,163]$. It is also believed that proximity to a critical point may actually stabilize novel ground state like high $\mathrm{T}_{\mathrm{c}}$ superconductors in itinerant systems $[163,164]$. These properties raise 


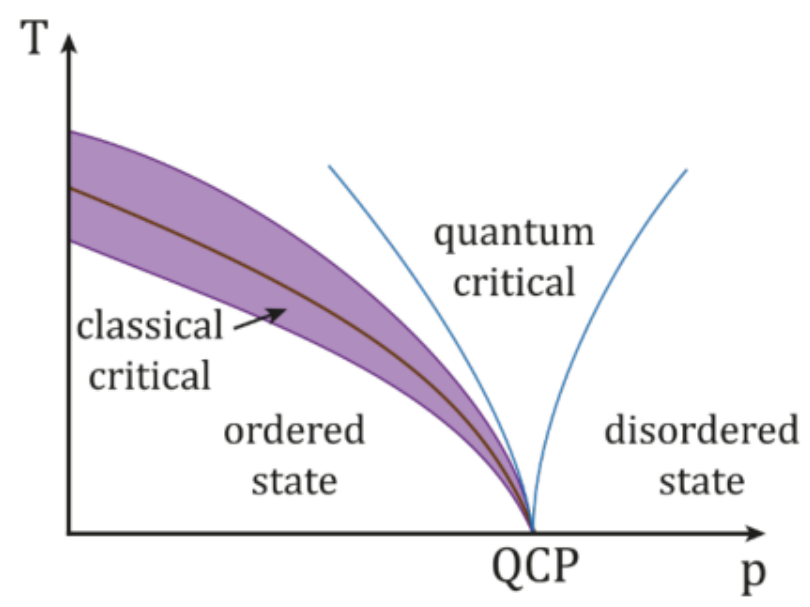

Figure 6.1: Schematics of a second order phase transition showing different phases as a function of some tuning parameter $p$ with temperature. The funnel shaped middle part is where the effect of the $T=0$ QCP is still felt at higher temperatures. The ordered state on the left has a broken symmetry [Source: Wikipedia]

the urgency to understand the details of the mechanisms that drive such phase transitions, characterize them and investigate whether such possibilities might indeed be true.

On a separate note, the Fermi liquid theory is one of the important tools for the experimental determination of the properties of a material. The low temperature properties of metals are often understood in terms of this famous theory. Despite having strong many-body interactions present, using this theory one understands the properties of such metals to be derived from "quasiparticles". One key element of this theory is that such quasiparticle excitations of the Fermi sea carry the same charge, spin etc. as the electrons constituting them, while having a different, generally larger renormalized effective mass $m^{*}$. Such a mass enhancement in the so-called heavy-fermion systems is sometimes understood as an outcome of this mentioned 
many-body interactions.

However, as one approaches a quantum critical point by tuning some parameter as described above, this theoretical cornerstone to understand such low temperature properties breaks down. Signatures for such violations are often recognized by a diverging $m^{*}$ or by deviations from the quadratic temperature dependent power law of electrical resistivity. Divergence in other transport properties like the specific heat or magnetic susceptibility is also observed. Such inconclusive nature of these properties once again urges for a thorough understanding of the low-temperature physics of strongly-correlated materials and it becomes necessary to understand its responsible mechanisms (e.g. enhanced electron scattering off the fluctuations or even quasiparticle-quasi-particle scattering) and how such deviations from the Fermi-liquid theory can be understood in the context of QCP.

\section{2 $\mathrm{Sr}_{3} \mathrm{Ru}_{2} \mathrm{O}_{7}:$ Quantum criticality and its proper- ties}

$\mathrm{Sr}_{3} \mathrm{Ru}_{2} \mathrm{O}_{7}$, as mentioned above, first came to attention for showing the signatures of a QCP in the vicinity of a first-order metamagnetic transition. A metamagnetic transition is characterized by the sudden non-linear rise in magnetization of a material at some finite field. Since the symmetry of the initial and final magnetic phases stays the same in such a transition, it must be either a first order phase transition, with the consequent discontinuous jump in the magnetization, or simply a crossover. At 
first sight, neither of these can be a source of the required diverging susceptibilities etc. (as shown later in figure 6.2 and figure 6.3). However, due to this symmetry conservation between the two phases, it is possible for this first order line to end at a critical endpoint (CEP). This CEP shares the property of a second order phase transition that is the key to producing quantum criticality, since it is characterized by diverging susceptibilities and the physics is dominated by fluctuations. The main qualitative difference is the absence of any spontaneous breaking of symmetry [165]. In this subsection, the literature review spanning the investigation of the nature of quantum criticality and its other interesting properties have been presented.

Early experiments e.g. by Cao et al. [166] found this material to have a rich ferromagnetic ground state of the itinerant (and probably canted) nature carrying saturated moments of around $1.3 \mu_{\mathrm{B}}$ per $\mathrm{Ru}$ atom with Curie temperature $T_{\mathrm{c}}$ of $104 \mathrm{~K}$. Owing to the phase impurity of the metal, soon Huang et al. [167] reported $\mathrm{Sr}_{3} \mathrm{Ru}_{2} \mathrm{O}_{7}$ to have no magnetic order down to $1.6 \mathrm{~K}$. It was soon confirmed by Ikeda et al. [168] where they concluded it to be a Fermi-liquid paramagnet with a high (i10) Wilson ratio. Although axial pressure variations normal to the $\mathrm{RuO}_{2}$ planes were later found to revert this back to ferromagnetism suggesting it to be in proximity of a ferromagnetic instability [169]. Later, using inelastic neutrons scattering, Capogna et al. [170] also found FM spin fluctuations present over $T \geq 20 \mathrm{~K}$.

By 2001, Perry et al. [172] and Grigera et al. [163] observed critical fluctuations and metamagnetism. With their thorough investigation, $\mathrm{Sr}_{3} \mathrm{Ru}_{2} \mathrm{O}_{7}$ was established to feature a new class of quantum critical point arising when the critical end-point 
of this first order metamagnetic transition was depressed down towards absolute zero using magnetic field of about 7.8 $T$ strength (figure 6.2). It was confirmed and refined further again by Grigera et al. [173] where they observed that the first order end-point sits at about $1.25 \mathrm{~K}$ for in-plane magnetic fields. Aligning the field within $10^{\circ}$ of the $c$ axis depressed it below $50 \mathrm{mK}$.

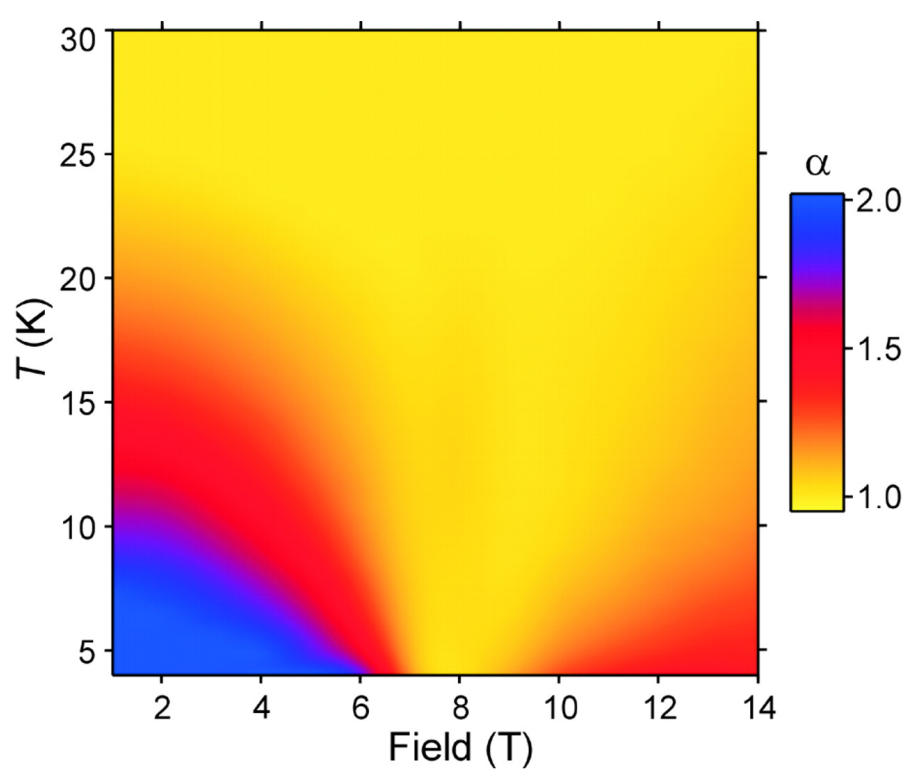

Figure 6.2: Reported phase diagram of $\mathrm{Sr}_{3} \mathrm{Ru}_{2} \mathrm{O}_{7}$ in terms of the temperature dependent power-law coefficient $(\alpha)$ of electrical resistance $(\rho)$ as a function of the magnetic field as the tuning parameter at low temperatures. The striking similarity to figure 6.1 is evident. A quadratic temperature dependence confirms its low $T$, low $B$ Fermiliquid behavior. Note how $\alpha=1$ (non-fermi liquid) regime depresses down towards zero with increasing field. $\alpha$ rises slowly on the other side of high $B$ limit [163]

It is worth noting here that the previous experimental investigations $[166,167,168]$ reported a tetragonal structure of $\mathrm{Sr}_{3} \mathrm{Ru}_{2} \mathrm{O}_{7}$. However, Saked et al. [174] found inplane $\mathrm{RuO}_{6}$ octahedral rotations by about $7^{\circ}$ which was soon confirmed by Kiyanagi et al. [175] to be accompanied by a nanometre-size twin orthorhombic distortion in 


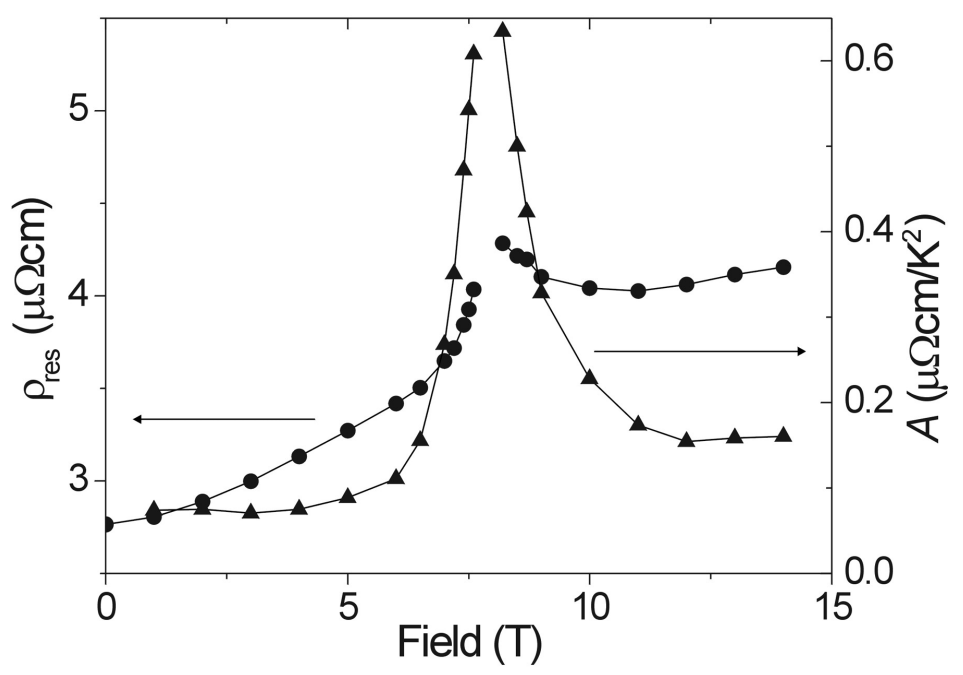

Figure 6.3: Characterizing the sharp change in $\rho\left(=\rho_{\text {res }}+\mathrm{AT}^{\alpha}\right)$ as a divergence of the quantity $A$ when the field sweeps in the vicinity of the metamagnetic field of magnitude $7.85 \pm 0.05 \mathrm{~T}$. Another metamagnetic transition occurs at $\mathrm{B} \sim 5.5 \mathrm{~T}$ for an in-plane $(a b)$ field direction [163].

the $a b$ plane. This is phenomenal for the material because such a distortion now makes the electronic structure of $\mathrm{Sr}_{3} \mathrm{Ru}_{2} \mathrm{O}_{7}$ different from its previous $(n=1)$ RuddlesdenPopper (RP) series member $\mathrm{Sr}_{2} \mathrm{RuO}_{4}$ by more than the mere addition of a double layering extra octahedron. This can be expected to cause subtle changes in the Fermi surface. The relevant crystal structures have been shown later in figure 6.5. Considering this distortion, such a possibility was pointed out by electronic structure calculations by Singh et. al [176]. It was also noted how this rotation is expected to reduce the in-plane $\mathrm{Ru}-\mathrm{Ru}$ hopping, and hence increase the density of states at the Fermi level, which may enhance the magnetic fluctuations.

In search of the existence for this metamagnetic transition related QCEP and its explanation, researchers also explored the possibility of Fermi surface instabilities in $\mathrm{Sr}_{3} \mathrm{Ru}_{2} \mathrm{O}_{7}$, specifically for van Hove singularities [177]. Theory and combined exper- 
imental investigation by Grigera et al. [178] suggested the presence of a new phase of this material which is strongly disorder (or sample purity) dependent and that the fluctuations associated with an itinerant metamagnetic QCP are rather unusual in the sense that they are fluctuations of the Fermi surface itself. In 2005, Kee and Kim [179] showed that metamagnetic transition in metals with a spin-dependent Fermi surface instability can occur via the formation of electronic nematic order, details of which has been explored in the next chapter.

In 2012, however, Mesa et al. [182], reported neutron scattering results on $\mathrm{Sr}_{3}\left(\mathrm{Ru}_{1-\mathrm{x}} \mathrm{Mn}_{\mathrm{x}}\right)_{2} \mathrm{O}_{7}$ where they observed the unusual E-type AFM order stabilized by $\mathrm{Mn}$ doping corresponding to $x=0.16$. Such an AFM order was found to exhibit only a single-bilayer thickness correlation along the $c$-direction. They concluded the presence of an unusual competition between AFM and FM order in the material since below $\mathrm{T}_{\mathrm{N}}=78 \mathrm{~K}$ it also exhibited similar single bilayer FM correlations. The resulting new phase space that has the doping concentration as the tuning parameter as reported has been shown in figure 6.4 [182]. Interestingly, the crossover point between the mentioned magnetic orders is at $x=0.16$ which is also where the octahedral rotation rapidly diminishes to zero.

\subsubsection{Introduction}

Quantum criticality, especially in the context of its material-dependent signatures, is of significant current interest $[183,184]$. Here, we investigate the competing orders present in the quantum critical metamagnet $\mathrm{Sr}_{3} \mathrm{Ru}_{2} \mathrm{O}_{7}[163,185,186,187]$. We find 


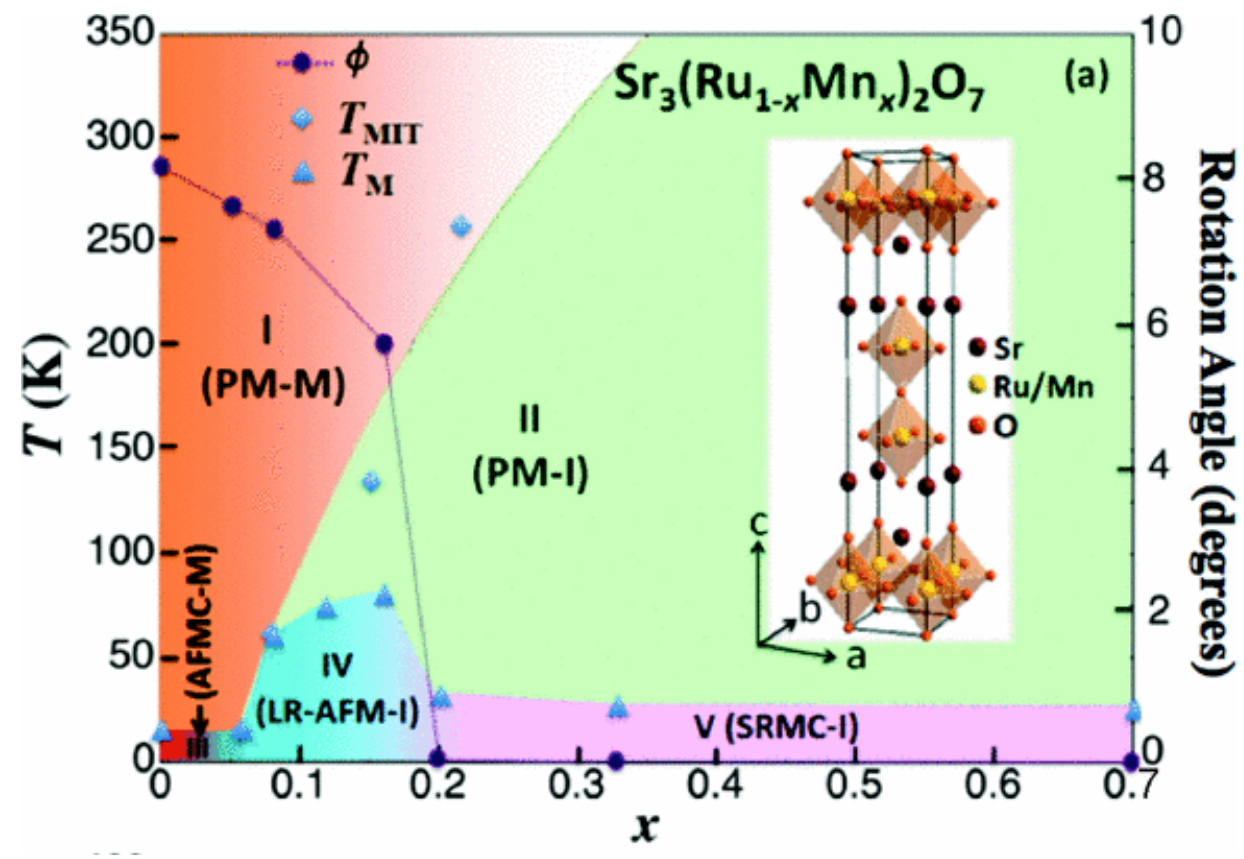

Figure 6.4: The schematic phase diagram of $\mathrm{Sr}_{3}\left(\mathrm{Ru}_{1-\mathrm{x}} \mathrm{Mn}_{\mathrm{x}}\right)_{2} \mathrm{O}_{7}$ as a function of $\mathrm{Mn}$ doping concentration. Region I is a paramagnetic metallic (PM-M) phase; region II is a paramagnetic insulating (PM-I) phase; region III is a metallic phase with AFM correlation (AFMC-M); and region IV is a long-range AFM insulating phase (LRAFM-I). The unit cell (with square lattice dimensions) has been shown in inset for reference. [182].

low-energy antiferromagnetically ordered states that energetically compete with ferromagnetism. Interestingly, we further find that these low-energy antiferromagnetic (AFM) states show substantial in-plane transport anisotropy, which we discuss in relation to nematicity in the next chapter.

Members of the Ruddlesden-Popper (RP) series of strontium ruthenate compounds, $\mathrm{Sr}_{\mathrm{n}+1} \mathrm{Ru}_{\mathrm{n}} \mathrm{O}_{3 \mathrm{n}+1}$, have many interesting characteristics. The $n=\infty$ member $\mathrm{SrRuO}_{3}$ is a rare $4 d$ itinerant ferromagnet [188, 189]. The $n=1$ member $\mathrm{Sr}_{2} \mathrm{RuO}_{4}$, however, is a known unconventional superconductor $[190,191,192]$. The $n=2$ 
bilayer compound, $\mathrm{Sr}_{3} \mathrm{Ru}_{2} \mathrm{O}_{7}$, the focus of the present work, shows quantum criticality under magnetic field. Its phase diagram shows a metamagnetic transition with a critical point that can be tuned to near zero temperature by applying magnetic field $[163,193,173,194,195]$. Borzi and co-workers reported a strong in-plane conductivity anisotropy in this near tetragonal compound around the critical point and characterized it as nematic $[181,180]$. More broadly, $\mathrm{Sr}_{3} \mathrm{Ru}_{2} \mathrm{O}_{7}$ presents an interesting case of a nearly ferromagnetic (FM) $4 d$ material with a layered crystal structure and considerable tunability of properties [175, 196, 166, 168, 197, 198, 199, 200, 201, 202, 203].

In general, various low-temperature properties of a system situated near a magnetic quantum critical point (QCP), including transport, are strongly influenced by its associated spin fluctuations, sometimes up to relatively high temperatures $[170,171,174,204]$. This is the case in $\mathrm{Sr}_{3} \mathrm{Ru}_{2} \mathrm{O}_{7}$, implying that the spin fluctuations associated with the critical point are relatively strong in this material. The underlying quantum fluctuations also lead to a suppression of magnetic order [205]. In addition, they also present challenges to the characterization of such systems [206]. Commonly employed density functional theory (DFT) approximations, such as the local density approximation (LDA), behave like a mean-field theory in this regard and do not capture the effect of such spin fluctuations that arise near a quantum critical point [207]. These large fluctuations lead to a systematic overestimation of ground state magnetizations in DFT calculations [208, 209].

We note that the overestimation of magnetizations and magnetic moments in standard density functional calculations for materials is unusual. In weak and moderately 
correlated magnetic materials standard DFT yields generally good agreement with experiment. This includes materials such as the $3 d$ ferromagnets $(\mathrm{Fe}, \mathrm{Co}, \mathrm{Ni}$, and a wide variety of intermetallics based on them) $[210,211,212]$, as well as the ferromagnetic perovskite $\mathrm{SrRuO}_{3}[188,213]$, which is chemically and structurally very similar to $\mathrm{Sr}_{3} \mathrm{Ru}_{2} \mathrm{O}_{7}$. In strongly correlated systems, such as Mott insulators, the moments are often strongly underestimated by standard DFT calculations. For example, in the undoped parents of the high-temperature cuprate superconductors, DFT calculations fail to produce the experimentally observed antiferromagnetic ground states [214]. In these systems, the Coulomb repulsion, which is needed to localize the electrons, is inadequately represented in standard DFT calculations. Adding an additional Hubbard $\mathrm{U}$ then improves the description, including reproduction of the ground state of undoped cuprates $[215,216]$.

While such strongly correlated materials, where standard DFT calculations underestimate magnetic ordering and do not properly describe the ground state, are relatively common, materials where such calculations overestimate the magnetic moments are much less common. These are cases where spin fluctuations, often associated with nearby quantum critical points, are strong enough to significantly reduce the bare DFT moments. This has been discussed in terms of a bare DFT energy surface as a function of magnetization that is then renormalized by spin fluctuations using a fluctuation amplitude and a fluctuation renormalized Landau theory analogous to lowest order self-consistent phonon theory [217, 218]. Applying this in a quantitative way to predict the renormalized magnetic properties from first principles is not straightforward due to the difficulty in determining a cutoff to distinguish 
spin fluctuations associated with the critical point, not included in standard DFT, from higher-energy spin fluctuations that are included [208]. However, by comparing standard DFT calculations with experiment, estimates have been made of fluctuation amplitudes [208, 209]. Not surprisingly, the addition of Coulomb correlations by methods such as LDA $+U$ degrades agreement with experiment in these cases since it introduces shifts opposite to those needed [219]. Furthermore, the magnitude of this type of deviation between DFT and experiment has been used as a signature to identify materials near magnetic quantum critical points [209, 220, 221, 222], including successful predictions confirmed by subsequent experiments, as in the cases of hydrated $\mathrm{Na}_{\mathrm{x}} \mathrm{CoO}_{2}$ and $\mathrm{YFe}_{2} \mathrm{Ge}_{2}$ [223, 224, 225, 226, 227, 228, 229].

It is also of interest to note the connection of $\mathrm{Sr}_{3} \mathrm{Ru}_{2} \mathrm{O}_{7}$ and its magnetism to other members of the RP series, $(\mathrm{Sr}, \mathrm{Ca})_{\mathrm{n}+1} \mathrm{Ru}_{\mathrm{n}} \mathrm{O}_{3 \mathrm{n}+1}$. As mentioned, $\mathrm{SrRuO}_{3}$ is a ferromagnet [230] with itinerant character that is well described by LDA calculations as far as its magnetism is concerned [188, 231, 232, 233]. Furthermore, details of its electronic structure, including, for example, LDA-based predictions of a negative spin polarization, have been confirmed in detail by experiments [234, 235, 236].

Theoretical work indicates significant sensitivity of the magnetism to structure in this compound [231, 237, 238]. Experimentally, alloying with Ca leads to increased distortion of the ideal perovskite structure through octahedral tilts. This is accompanied by a decrease in the magnetic ordering temperature until a critical point is reached at $\sim 70 \% \mathrm{Ca}$, beyond which a highly renormalized near ferromagnetic metal is found [239, 240]. 
The importance of octahedral tilts and rotation in relation to magnetism is also found in single-layer $(\mathrm{Sr}, \mathrm{Ca})_{2} \mathrm{RuO}_{4} \cdot \mathrm{Sr}_{2} \mathrm{RuO}_{4}$ is a paramagnetic Fermi liquid that exhibits unconventional superconductivity at low temperature [190, 192]. There has been debate about the extent and nature of correlations in this material $[241,242,243$, 244]. However, it is generally agreed that the Fermi surface agrees with that predicted by LDA calculations [245, 246], although with mass renormalization [247, 248], that spin fluctuations likely play an important role in the superconductivity [213, 249, 250] and that these spin fluctuations have a substantial itinerant origin. This itinerant behavior includes the observation of incommensurate spin fluctuations predicted on the basis of Fermi-surface nesting [251]. Alloying with $\mathrm{Ca}$ in $(\mathrm{Sr}, \mathrm{Ca})_{2} \mathrm{RuO}_{4}$ again demonstrates sensitivity to structure. Initially there is an increasing ferromagnetic susceptibility as the octahedra rotate, followed by a crossover, and eventually near pure $\mathrm{Ca}_{2} \mathrm{RuO}_{4}$ the development of an antiferromagnetic insulating phase with a strong change in the Ru-O bond lengths reflecting distortion of the octahedra [252, 253].

In any case, the fluctuation-dissipation theorem, which relates the amplitude of the fluctuations to the dissipation, given by an integral involving the imaginary part of the susceptibility, implies an enhanced imaginary component of the magnetic susceptibility associated with the sizable fluctuations in materials near magnetic quantum critical points. This in turn points towards the presence of strongly competing orders in materials that show strong spin fluctuations but no order, as discussed previously [218]. Besides an overly strong tendency towards ferromagnetism, both FM and AFM fluctuations $[170,254]$ may coexist in this ruthenate system $[171,250]$. Here we report 
a search for such competing orders including commonly discussed AFM states as well as the so-called E-type order that occurs with heavy Mn doping [182].

\subsubsection{Computational Methods}

We searched initially for various possible magnetic orders using projector augmented wave (PAW) pseudopotentials as implemented in the Vienna Ab initio Simulation Package (VASP) [24, 25]. An energy cutoff of $500 \mathrm{eV}$ was used. Energy and force convergence criteria were chosen as $10^{-7} \mathrm{eV}$ and $0.01 \mathrm{eV} \AA^{-1}$, respectively. The Brillouin zone (BZ), in this case, was sampled on a $5 \times 5 \times 5 \mathrm{k}$ mesh. We used both LDA and the Perdew-Burke-Ernzerhof (PBE) generalized gradient approximation [17]. We also checked the structural predictions of these two functionals. We find that the PBE functional leads to a unit cell volume 1.6\% larger than experiment (average lattice parameter error of $+0.5 \%$ ), while the LDA leads to an underestimate of the unit cell volume by $6.6 \%$ (average lattice parameter error of $-2.2 \%$ ). These are within the range of typical errors for these functionals and the somewhat smaller lattice parameters predicted by LDA relative to PBE is also as usual.

Following this survey, we then investigated the low-lying states in detail using the general potential linearized augmented plane wave (LAPW) [6] method as implemented in the WIEN2k code [5]. The LAPW sphere radii for Ru and Sr atoms were both chosen as RMT=2.1 Bohr, while for O atoms RMT=1.55 Bohr was used. The basis size was set by plane-wave cutoff $K_{\max }$ with $R_{\min } K_{\max }=7.0$. This leads to an effective $\mathrm{RK}_{\max }=9.5$ for the metal atoms. The self-consistent calculations were 
performed using a BZ sampling of at least $1000 \mathrm{k}$ points in the respective BZs. Transport integrals were done using the BoltzTraP code [7]. Dense Brillouin zone sampling with $\mathbf{k}$ meshes of dimensions $30 \times 16 \times 16$ or higher was used for these calculations.

$\mathrm{Sr}_{3} \mathrm{Ru}_{2} \mathrm{O}_{7}$ has a layered perovskite structure that is formed by two sheets of cornersharing $\mathrm{RuO}_{6}$ octahedra connected via a shared apical oxygen (figure 6.5). Interestingly, the metal ions still occupy the ideal tetragonal symmetry sites similar to the $n$ $=1$ compound $\mathrm{Sr}_{2} \mathrm{RuO}_{4}$, although the $\mathrm{Ru}-\mathrm{O}-\mathrm{Ru}$ bonds are bent due to the counterrotation of the octahedra about the $c$ axis. These rotations amount to approximately $7^{\circ}$ and are opposite for the two sheets making up a bilayer. This in combination with the stacking of the bilayers reduces the overall symmetry so that finally the compound has an orthorhombic crystal structure, space group Ccca (No. 68) [174, 255, 167, 256].

The lattice parameters for our calculations were taken from experiment, specifically the measurements performed on single crystals, as reported by Kiyanagi et al. [175]. These are $a=5.4979 \AA, b=5.5008 \AA$, and $c=26.7327 \AA$. The internal positions of the atoms were relaxed. Details of the structure are given below:

\subsubsection{Results and discussion on the magnetic order}

$\mathrm{Sr}_{3} \mathrm{Ru}_{2} \mathrm{O}_{7}$ is a known metal and despite having a susceptibility peak at around $\sim 18 \mathrm{~K}[197,167]$, it displays no long-range magnetic order [168]. Multiple reports, however, suggest temperature-dependent competing FM and AFM spin fluctuations, although the nature of the AFM fluctuations has not been established $[201,170,172,257,258,259,260]$. 


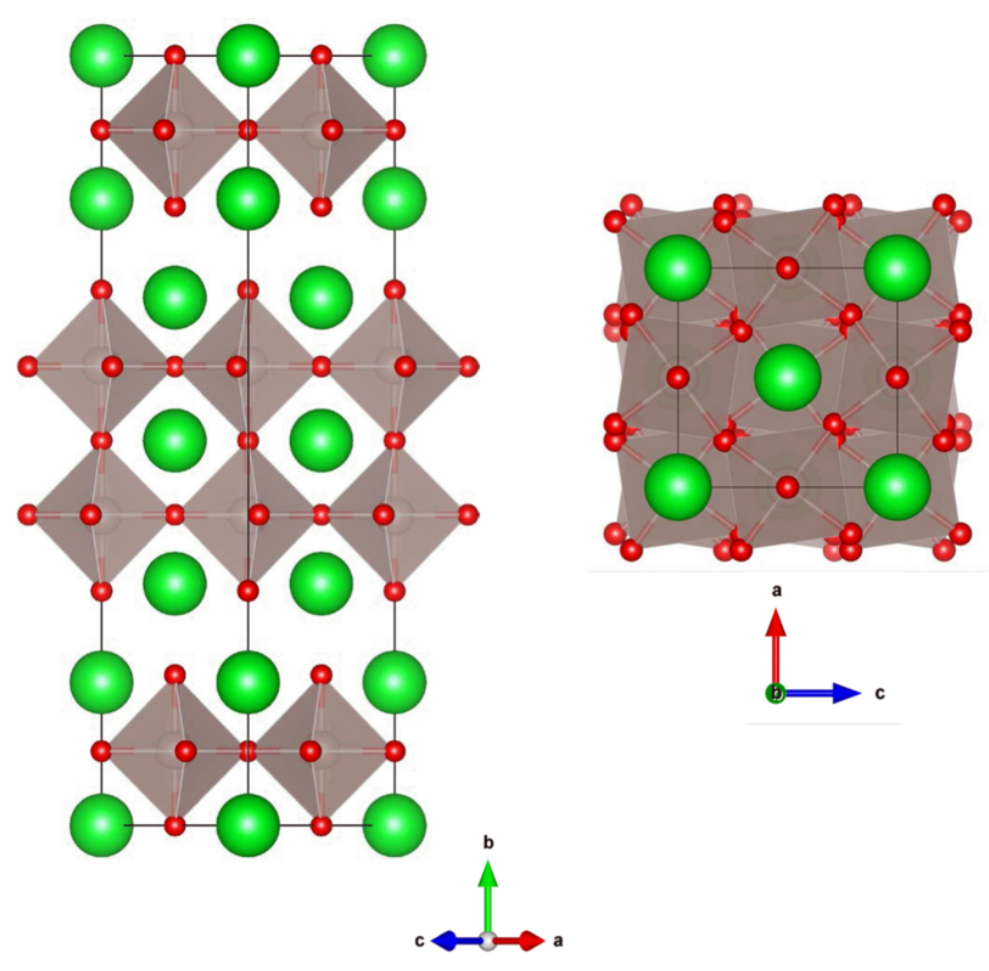

Figure 6.5: Crystal structure of orthorhombic $\mathrm{Sr}_{3} \mathrm{Ru}_{2} \mathrm{O}_{7}$, showing layering along $c$ (left) and the view along the $c$ axis, illustrating octahedral rotations (right).

Table 6.1: Relaxed atomic coordinates for $\mathrm{Sr}_{3} \mathrm{Ru}_{2} \mathrm{O}_{7}$. Coordinates are given in the standard setting, $C c c e$, origin choice 2 for spacegroup number 68 . The lattice parameters from experiment (see text) with this setting are, $a=5.4979 \AA, b=20.7327$ and $\AA$, $c=5.5008 \AA$.

\begin{tabular}{cccc}
\hline \hline Site & $x$ & $y$ & $z$ \\
\hline Ru $8 f$ & 0.0000 & 0.1517 & 0.7500 \\
Sr 4a & 0.0000 & 0.7500 & 0.7500 \\
Sr $8 f$ & 0.0000 & 0.9367 & 0.7500 \\
O $4 b$ & 0.0000 & 0.2500 & 0.7500 \\
O $8 f$ & 0.0000 & 0.0534 & 0.7500 \\
O $16 i$ & 0.2815 & 0.8473 & 0.7500 \\
\hline \hline
\end{tabular}

Both experimental and theoretical investigations show that the material lies close 
to a magnetic instability $[168,198,261]$. Apart from applying an external magnetic field along various directions, investigations by perturbing the system via uniaxial pressure [255, 262, 263], doping by both magnetic impurities [198, 199] and nonmagnetic impurities [257, 264] find various magnetic behaviors. DFT investigations find a ferromagnetic instability in contrast to the experimentally observed enhanced paramagnetic state $[168,176,265]$. As mentioned, this type of error is a characteristic of a material near a quantum critical point. In the case of $\mathrm{Sr}_{3} \mathrm{Ru}_{2} \mathrm{O}_{7}$, the predicted ferromagnetism has an itinerant origin, coming from a high density of states associated with the structure of the $\mathrm{t}_{2 g}$ bands, specifically Van Hove singularities in the $\mathrm{d}_{x y}$ band. This Stoner mechanism is similar to $\mathrm{SrRuO}_{3}$ and $\mathrm{Sr}_{2} \mathrm{RuO}_{4}$ [200, 213, 231, 176, 266]. The finding of an incorrect ferromagnetic state is not affected by spin-orbit coupling. In our calculations, which were done in a scalar relativistic approximation, we found a spin magnetization of $4.7 \mu_{\mathrm{B}}$ per unit cell (four Ru atoms, including the interstitial and $\mathrm{O}$ components) in the LDA, which is reduced by less than $10 \%$ to $4.4 \mu_{\mathrm{B}}$ per cell when spin orbit is included. Adding Coulomb correlations using the $\mathrm{LDA}+U$ method [216], with a moderate value $U=4 \mathrm{eV}$ and the standard fully localized limit double counting strongly increases the magnetization to $7.9 \mu_{\mathrm{B}}$ per cell, opposite to what is needed to produce agreement with experiment. This is not unexpected, since degradation of standard DFT results with the addition of $U$ has been noted in other itinerant magnetic systems previously [212, 267]

As mentioned, $\mathrm{Sr}_{3} \mathrm{Ru}_{2} \mathrm{O}_{7}$ displays a metamagnetic transition at a field strength of approximately 7-8 T [185]. However, doping using magnetic impurities [268] such as Fe [198] and Mn [199, 202, 202, 269, 270] as well as nonmagnetic Ti [264] has 
been found to yield different AFM orders [269]. In general, the relationship between these and the properties of the undoped compound is unclear, since these dopants produce strong perturbations of the system. Nonetheless, one notable order is the double stripe $E$-type order that is observed with heavy Mn doping [182], although it is reported to have a short correlation length $[198,182]$. It is to be noted that in particular it is quite unclear that the E-type order found in Mn-doped samples is reflective of the properties of undoped $\mathrm{Sr}_{3} \mathrm{Ru}_{2} \mathrm{O}_{7}$. This is because the Mn doping is also accompanied by sizable distortions in the crystal structure along with bandwidth changes that may stabilize antiferromagnetic structures [271, 272]. Furthermore, doping in $\mathrm{Sr}_{3} \mathrm{Ru}_{2} \mathrm{O}_{7}$ is often accompanied with transition to a state of more insulating transport $[198,199,182,264,271,273]$, while pristine $\mathrm{Sr}_{3} \mathrm{Ru}_{2} \mathrm{O}_{7}$ is clearly metallic and itinerant. This has led to a focus on other orders as possible competing orders to ferromagnetism in pristine $\mathrm{Sr}_{3} \mathrm{Ru}_{2} \mathrm{O}_{7}$. For example, based on their hybrid functional calculations, Rivero and co-workers reported other AFM orders both of metallic and insulating nature that may be obtained via strain, particularly a layered $A$-type AFM $[262,274,275,276]$.

Here we performed a search for possible long-range AFM orders (within collinear magnetism) in relation to both FM and nonmagnetic orders initially through PAW calculations (figure 6.6). We find the FM state as the ground state for both LDA and PBE functionals. Besides the FM order, the lowest energies are for the $E$-type order. There are two such states that are slightly nondegenerate due to orthorhombicity. PBE shows stronger magnetism over LDA including larger magnetic energies and higher moments. The three other commonly discussed AFM states lie much 
higher in energy in the order, $A<C<G$ (figure 6.6). No self-consistent solution was found for the $G$-type order within the LDA. The bottom six $S$-labeled AFM orders lie somewhere in the middle of the whole range. Details of the magnetic energies as obtained from these initial PAW calculations are shown below in figure 6.7 and table 6.3
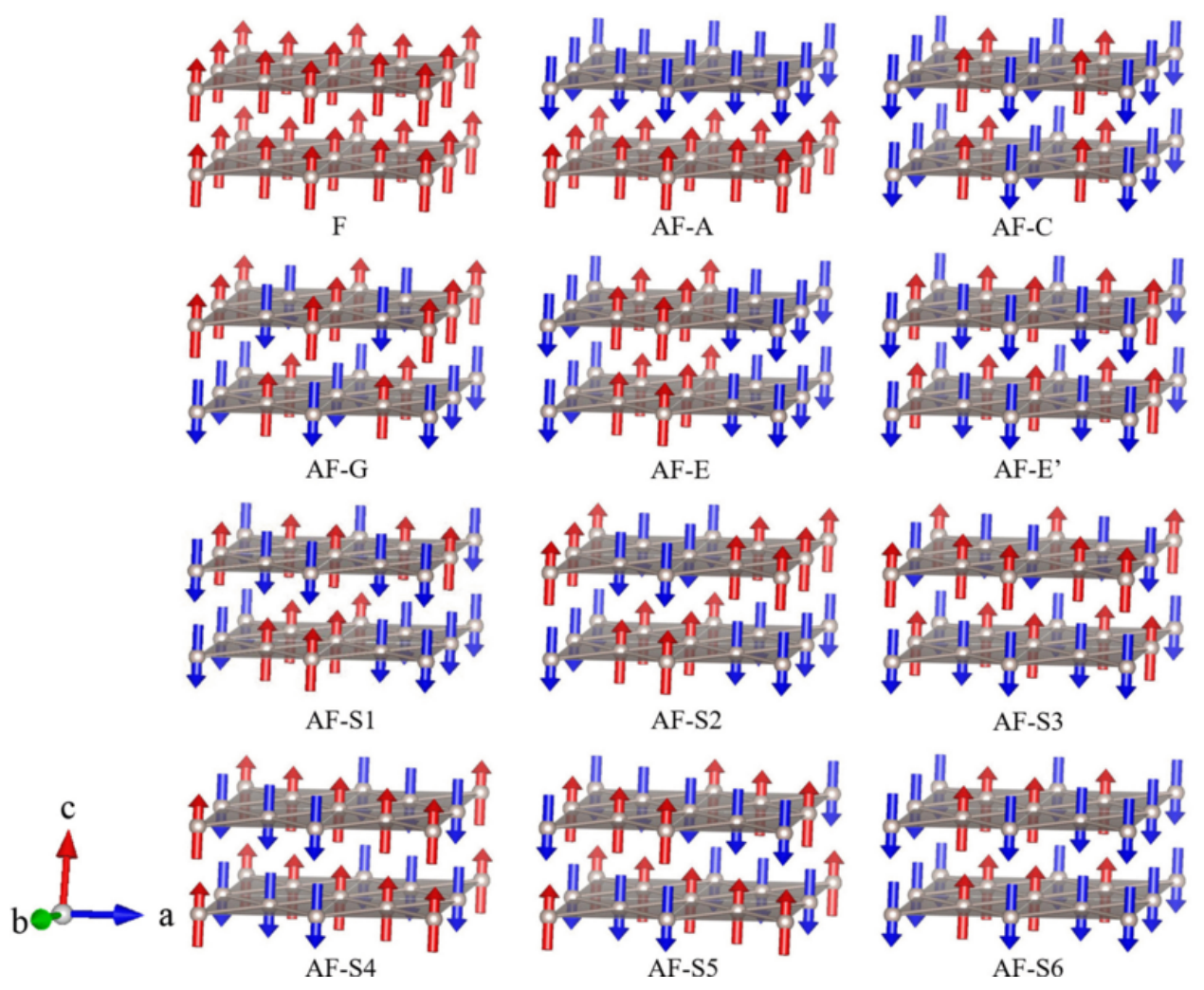

Figure 6.6: Various magnetic configurations investigated. Only Ru atoms are shown. These include ferromagnetism, $A$-type, $G$-type and $C$-type antiferromagnetism, which are common orders for perovskites, two $E$-type orders, which are slightly nondegenerate due to the orthorhombic crystal symmetry, and more complex orders with larger unit cells.

The lack of solution for $G$ type and the variability of the moments between the different states is a characteristic of itinerant magnetism, as is the fact that the energy differences between different orders are of similar order to the energy difference 
between the non-spin-polarized and the lowest-energy FM state. Thus local moment pictures, such as short-range Heisenberg models, are not well suited to this material. Furthermore, the $A$-type order, which consists of oppositely aligned FM layers stacked across the bilayer, lies much higher in energy than both of the $E$ types and the FM order. This means that interactions between the layers within a bilayer are strong.

We now turn to the detailed results obtained with the LAPW method $[201,6]$. The energetics and magnetic moments are in table 6.2. Most importantly, we find that the two metastable $E$-type AFM states carry large magnetic moments $\left(\sim 1.08 \mu_{\mathrm{B}}\right.$ within $\mathrm{PBE}$ and $\sim 0.85 \mu_{\mathrm{B}}$ within $\left.\mathrm{LDA}\right)$. As expected, these are the orders that consistently lie closest to the FM ground state.

Table 6.2: Energy ordering of various magnetic orders (per formula unit) found by LAPW calculations and their (absolute) averaged magnetic moments for both PBE and LDA functionals. Refer to figure 6.6 for naming. FM and nonmagnetic (NM) respectively stand for ferromagnetic and nonmagnetic state (zero-energy level), while all the other orders are antiferromagnetic in nature. Note that the moments reported here are those lying within the LAPW sphere radii.

\begin{tabular}{ccccc}
\hline \hline & \multicolumn{2}{c}{ PBE } & \multicolumn{2}{c}{ LDA } \\
\cline { 2 - 5 } Order & $\Delta E$ & Magn. mom. & $\Delta E$ & Magn. mom. \\
\cline { 2 - 5 } & $\mathrm{meV} /$ f.u. & $\mathrm{Ru}\left(\mu_{\mathrm{B}}\right)$ & $\mathrm{meV} /$ f.u. & $\mathrm{Ru}\left(\mu_{\mathrm{B}}\right)$ \\
\hline $\mathrm{FM}$ & -147.8 & 1.28 & -29.9 & 0.73 \\
$E$ & -131.5 & 1.08 & -28.6 & 0.85 \\
$E^{\prime}$ & -130.3 & 1.08 & -28.0 & 0.85 \\
$A$ & -95.6 & 1.06 & -21.2 & 0.60 \\
$C$ & -43.6 & 0.72 & -13.6 & 0.38 \\
$G$ & -3.2 & 0.45 & & \\
$\mathrm{NM}$ & 0 & 0.000 & 0 & 0.000 \\
\hline \hline
\end{tabular}

The sizable moments obtained and the FM ground state contradict the fact that 
$\mathrm{Sr}_{3} \mathrm{Ru}_{2} \mathrm{O}_{7}$ is an experimentally determined paramagnet. However, this is almost certainly due to the fact that the system lies close to a magnetic QCP where strong spin fluctuations suppress any long-range magnetic order in the system. Standard DFT calculations fail to describe this type of fluctuations, as has been noted for other such materials near a magnetic QCP [201, 209, 277, 278, 279]. As mentioned, this overestimation of magnetism has been used as a signature of materials that are in the vicinity of a QCP [208, 209, 218, 279].

In addition, one may note that the magnetic moments predicted for the $E$-type orders are indeed the largest among the AFM states. All the other investigated orders lie higher in energy and have lower magnetic moments. The energy difference within LDA between the FM and $E$ orders is only $1.6 \mathrm{meV}$ per formula unit on average (for the $E$ and $E^{\prime}$ ). Thus we find that the $E$-type order is very likely the order that competes with ferromagnetism in this material. It is interesting to note that the E-type order is also the order among the ones considered that breaks the tetragonal symmetry within the $\mathrm{RuO}_{2}$ plane.

Through these results we find the E-type striped AFM order is our lowest lyting metastable state, in energetically close competition with the FM ground state. In the next chapter, we will focus on the details of the electronic structure of this material and look upon transport properties. The implications of our transport results will then be discussed in relation to well known experimental results on electronic nematicity by Borzi et al. 
Table 6.3: Energetics and magnetic moments for 12 different magnetic orders as shown in figure 6.7 found using both LDA and the PBE functionals. FM and NM respectively stand for ferromagnetic and nonmagnetic orders.

\begin{tabular}{ccccc}
\hline \hline \multirow{2}{*}{ Order } & \multicolumn{2}{c}{ PBE } & \multicolumn{2}{c}{ LDA } \\
\cline { 2 - 5 } & $\Delta \mathrm{E}(\mathrm{meV})$ & $\begin{array}{c}\mathrm{Mag} \text { Mom. } \\
\mathrm{Ru}\left(\mu_{\mathrm{B}}\right)\end{array}$ & $\Delta \mathrm{E}(\mathrm{meV})$ & $\begin{array}{c}\text { Mag. Mom. } \\
\mathrm{Ru}\left(\mu_{\mathrm{B}}\right)\end{array}$ \\
\hline $\mathrm{FM}$ & -160.6 & 1.35 & -22.6 & 0.52 \\
$\mathrm{E}$ & -146.7 & 1.14 & -19.6 & 0.79 \\
$\mathrm{E}$, & -145.6 & 1.14 & -19.5 & 0.78 \\
$\mathrm{~A}$ & -106.8 & 1.13 & -17.3 & 0.48 \\
$\mathrm{C}$ & -49.4 & 0.77 & -1.7 & 0.35 \\
$\mathrm{G}$ & -14.2 & 0.48 & 0 & 0.00 \\
S1 & -87.4 & 1.16 & -14.8 & 0.84 \\
S2 & -85.2 & 0.95 & -6.1 & 0.51 \\
S3 & -84.9 & 0.95 & -6.1 & 0.51 \\
S4 & -108.2 & 1.12 & -16.3 & 0.59 \\
S5 & -67.1 & 0.97 & -5.4 & 0.40 \\
S6 & -49.5 & 0.85 & -10.4 & 0.52 \\
NM & 0 & 0 & 0 & 0 \\
\hline \hline
\end{tabular}



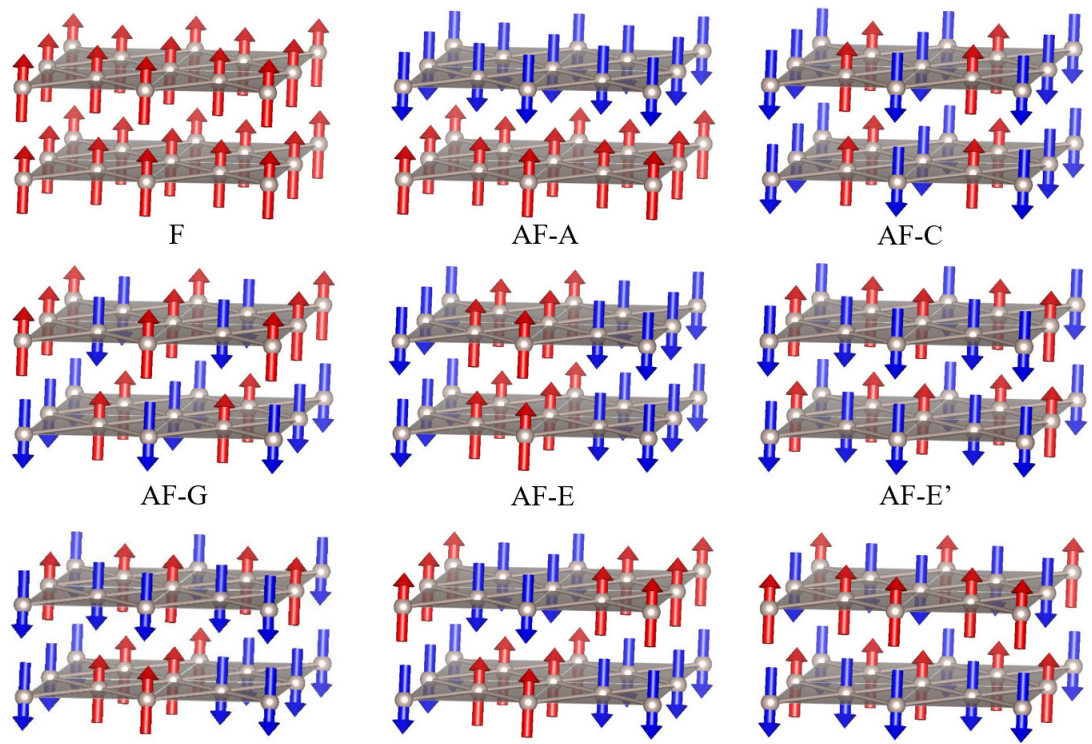

AF-E

AF-E'

AF-S1
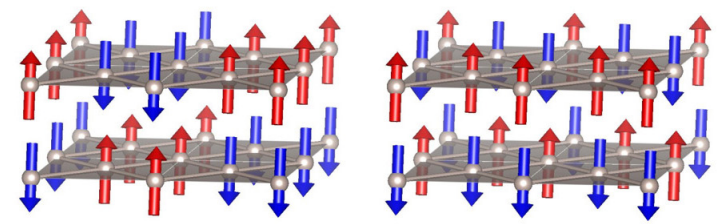

AF-S2

$\mathrm{AF}-\mathrm{S} 3$
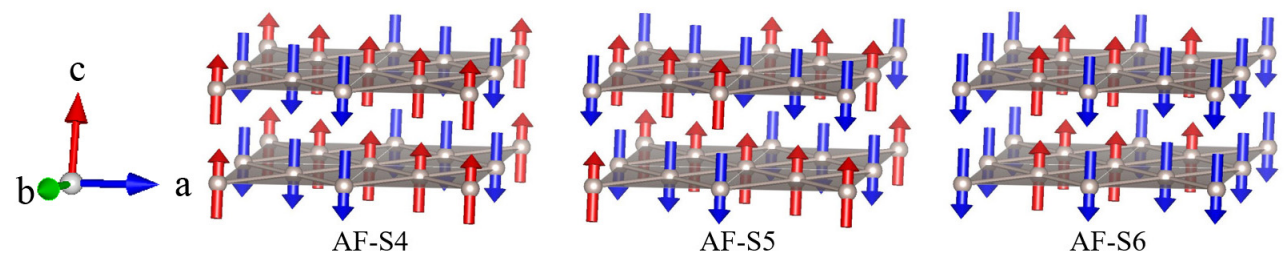

Figure 6.7: All the magnetic orders investigated in our PAW pseudopotential search strategy. Refer to 6.3 for the detailed energetics for each states.

\subsubsection{Summary}

In this chapter, I reviewed the quantum criticality phenomenon where a non-thermal parameter can be tuned to depress the transition temperature down towards zero kelvin. It was also noted how the low-temperature properties of a material near such a quantum critical point is dominated by strong fluctuations associated with it. It is thus imperative to understand the nature of these fluctuations which is closely related to the low-energy metastable states of the material itself.

Further, I studied the widely known quantum critical material: $\mathrm{Sr}_{3} \mathrm{Ru}_{2} \mathrm{O}_{7}$ where it 
was shown [163] that using magnetic field of strength around 8T, the transition temperature of the metamagnetic transition can be depressed down toward zero kelvin. This material, especially its magnetism, has been a subject of intense investigation, where the material was experimentally found to be paramagnetic. However, our firstprinciples DFT results find a ferromagnetic ground state within both LDA and PBE functionals. This is a known magnetic order disruption has been regularly seen in quantum critical material where these widely used density functionals fail to capture these strong inherent spin fluctuations. A previous doping study by Mesa et al. [182] found very weak E-type ordering in the narrow space of its rich magnetic phase diagram probed by $\mathrm{Mn}$ doping of $\mathrm{Sr}_{3} \mathrm{Ru}_{2} \mathrm{O}_{7}$.

Our first-principles results in search of these low-lying metastable states of this material using a two fold search strategy yielded that this striped $E$-type antiferromagnetically ordered state indeed does compete with its predicted ferromagnetic ground state. Due to its slight in-plane orthorhombic distortion from its closely related compound $\mathrm{Sr}_{2} \mathrm{RuO}_{4}$ structure, this gives rise to two separate slightly degenerate states (e.g.: $1.2 \mathrm{meV}$ per formula unit within PBE functional). The very high energy ordering of the other known magnetically ordered as well as the lack of G-type antiferromagnetism within LDA functional strongly suggests its magnetism to be of itinerant nature. The next chapter will focus onto the electronic structure of the material where this will be examined along with other interesting properties of this material. 


\section{Chapter 7}

\section{Electronic structure and transport anisotropy in $\mathrm{Sr}_{3} \mathrm{Ru}_{2} \mathrm{O}_{7}$ in relation to experimental observations}

In the previous chapter, the bilayered $\mathrm{Sr}_{3} \mathrm{Ru}_{2} \mathrm{O}_{7}$ was introduced. A well-known quantum critical material, its low temperature ground state properties are expected to be dominated by large fluctuations. As discussed earlier, the nature of these fluctuations are determined by its low-energy metastable states. We also noted how these strong fluctuations are also expected to disrupt any long range magnetic order present in the system. While bare density functional methods, within both LDA and GGA functionals predict its ground state to be of ferromagnetic nature, the results of our search for these lowest energy metastable states yielded two slightly non-degenerate antiferromagnetic $E$-type ordered states. Summarizing quantitatively, these are energetically within less than $20 \mathrm{meV}$ and $2 \mathrm{meV}$ per formula unit to the ground state, respectively. 
Previously, we discussed the metamagnetic transition and quantum criticality of this $\mathrm{Sr}_{3} \mathrm{Ru}_{2} \mathrm{O}_{7}$. Here, we focus on the property of electronic nematicity. An electronic nematic phase is defined when the rotational invariance is spontaneously broken and exhibits orientational (two-fold symmetry) order. One must note that this symmetry breaking is independent of the underlying crystal symmetries since it is exhibited by the strongly correlated electronic fluid only [181]. In 2005, Kee and Kim [179] reported that this metamagnetic transition in metals with a spin-dependent Fermi surface instability can occur via the formation of electronic nematic order. Later, Borzi et al. [180] confirmed the presence of such an electronic nematic fluid in high purity $\mathrm{Sr}_{3} \mathrm{Ru}_{2} \mathrm{O}_{7}$ crystals. It is interesting to note that their corresponding elastic neutron scattering could not detect any structural change within an upper limit of $10^{-5}$ in lattice anisotropy in connection to the formation of this nematic phase. For this, Borzi et al. postulated the presence of domains that cause extra scattering which quickly disappears as the field direction is tilted away from $c$-direction resulting in destruction of such domains. Later, in 2012, Mackenzie et al. concluded that the mechanism behind the formation of the nematic phase and its interrelation to quantum criticality and its metamagnetic jump is not fully understood [281]. In 2013, Stingl et al. in their contradicting study reported that for magnetic field variations ranging from below $7.8 \mathrm{~T}$, the system does retain four fold symmetry. This symmetry is broken for fields in nematic phase, i.e. of strength between $7.8 \mathrm{~T}$ and $8.1 \mathrm{~T}$ to a very small degree of 1 part in $10^{6}$. They also detected a small thermal expansion anisotropy for fields stronger than $8.1 \mathrm{~T}$ [282].

In figure 7.1, one may note that under a specific magnetic field, how the in-plane 


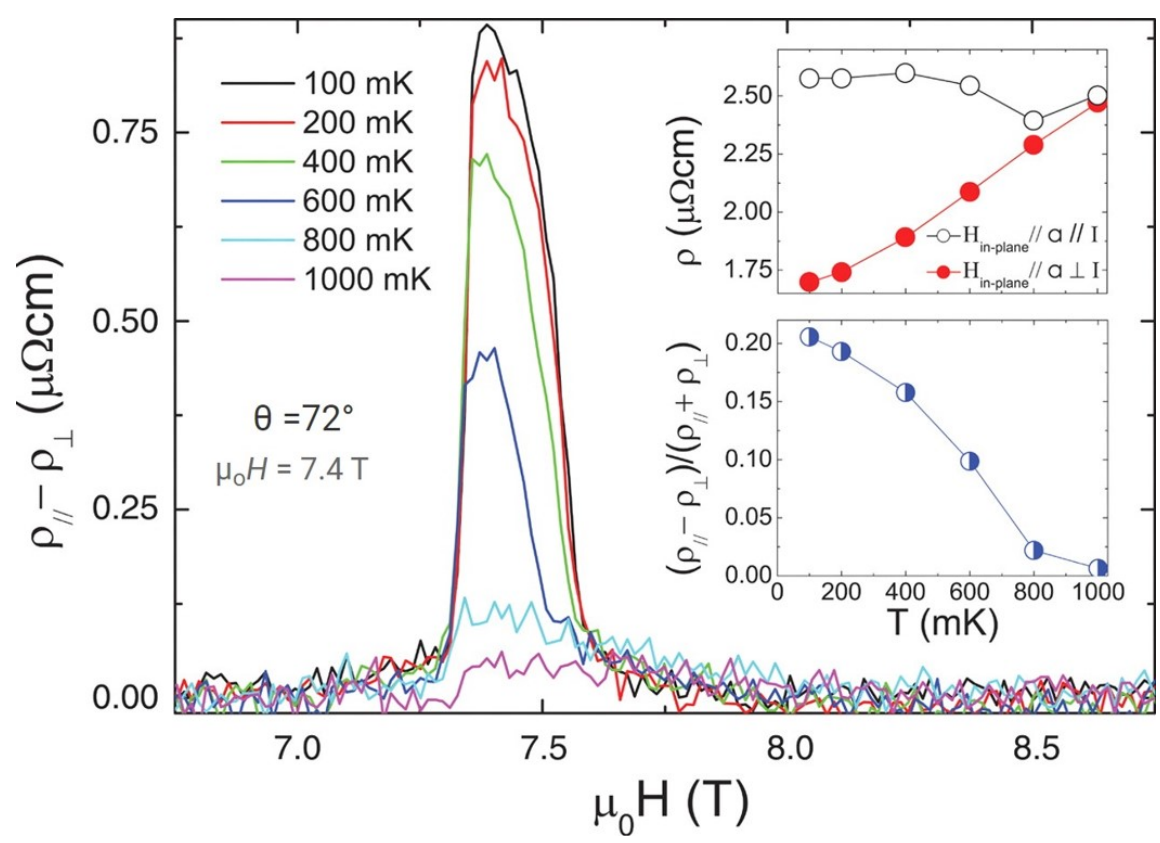

Figure 7.1: The temperature dependent difference in the in-plane resistivity $\rho_{\text {aa }}$ and $\rho_{\mathrm{bb}}$ for magnetic fields applied at $\theta=72^{\circ}$ where its in-plane component lies along $a$ direction. More details in ref. [180].

resistivity (magnetoresistivity) shows an ever-increasing anisotropy along both $a$ and $b$ axes with decreasing temperature. This exhibits the temperature dependent nematicity for $\mathrm{Sr}_{3} \mathrm{Ru}_{2} \mathrm{O}_{7}$. From the above literature review, one may conclude that the true nature of this nematic phase is not fully understood, especially in relation to the quantum critical behavior of $\mathrm{Sr}_{3} \mathrm{Ru}_{2} \mathrm{O}_{7}$. In the following section, we employ our known first-principles techniques and its derived transport calculations where we discuss the electronic structure of $\mathrm{Sr}_{3} \mathrm{Ru}_{2} \mathrm{O}_{7}$ and report out interesting results in terms of its observed in-plane anisotropy. 


\subsection{Electronic structure of $\mathrm{Sr}_{3} \mathrm{Ru}_{2} \mathrm{O}_{7}$}

We start our discussion of the with the density of states (DOS) of nonmagnetic $\mathrm{Sr}_{3} \mathrm{Ru}_{2} \mathrm{O}_{7}$, as shown in figure 7.2 shows a peak around the Fermi level. This favors magnetism through the Stoner mechanism as discussed previously [231]. For the considered $E$-type orders, the corresponding DOS (shown in the table 7.2 ) is distorted but still high near the Fermi level. Table 7.1 summarizes the DOS value observed at the Fermi level, $N\left(E_{F}\right)$ for each investigated magnetic order. Table 7.1 shows that the electronic structure remains metallic for all the spin orderings considered. As noted previously, there is strong $\mathrm{Ru} 4 d-\mathrm{O} 2 p$ hybridization evident. Table 7.2 shows the density of states as obtained through PAW pseudopotential method.

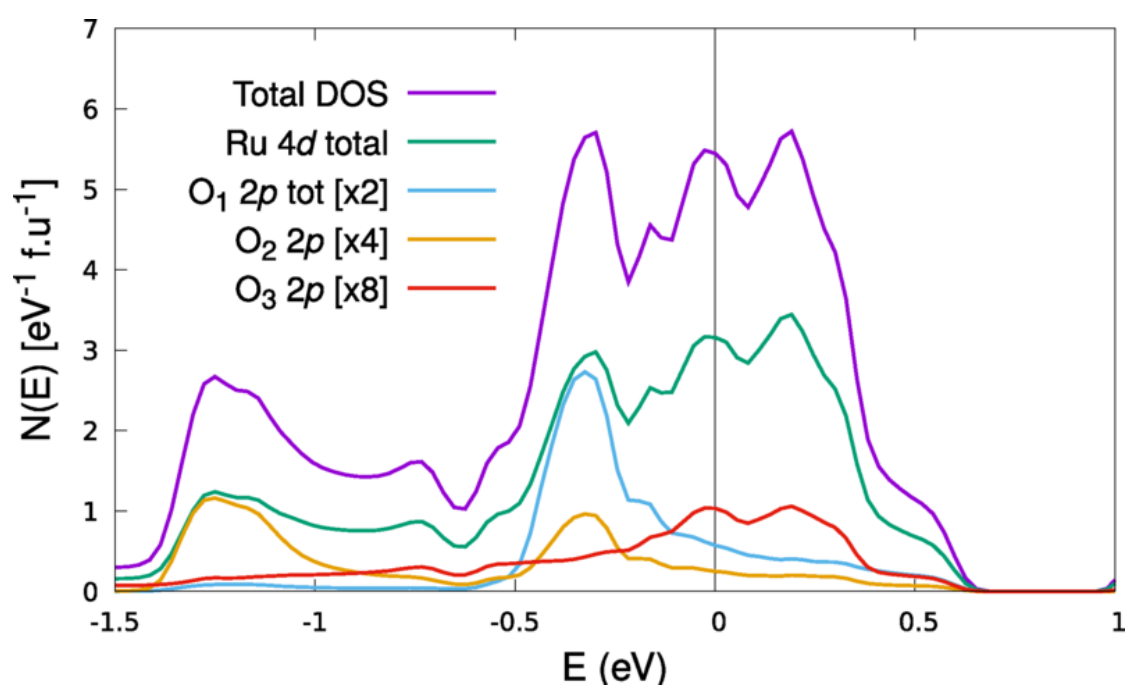

Figure 7.2: Density of states per formula unit of $\mathrm{Sr}_{3} \mathrm{Ru}_{2} \mathrm{O}_{7}$ for its nonmagnetic state showing the respective LAPW sphere projected contributions from $\mathrm{Ru} 4 d$ and $\mathrm{O}$ $2 p$ orbitals obtained within LDA. Three kinds of $\mathrm{O}$ contributions (scaled four times for visibility) are observed (see text) with their respective multiplicities, shown in parentheses. The black vertical line at $E=0$ shows the Fermi level. 
Table 7.1: Density of states (per formula unit) at the Fermi level, $N\left(E_{\mathrm{F}}\right)$ various magnetic orders with both LDA and PBE functionals. Note that for the ferromagnetic order $(\mathrm{FM})$, and for other magnetic orders, $N\left(E_{\mathrm{F}}\right)$ for each single spin channel $(\uparrow, \downarrow)$ is shown. A Gaussian broadening of $4 \mathrm{meV}$ was used. Units are $1 / \mathrm{eV}$.

\begin{tabular}{|c|c|c|}
\hline \multirow{2}{*}{ Order } & \multicolumn{2}{|c|}{ LAPW } \\
\hline & LDA & PBE \\
\hline $\mathrm{FM}(\uparrow, \downarrow)$ & $3.9 \quad 3.4$ & $0.9 \quad 4.8$ \\
\hline $\mathrm{E}$ & 5.0 & 4.5 \\
\hline$E^{\prime}$ & 5.4 & 4.7 \\
\hline $\mathrm{A}$ & 4.0 & 3.0 \\
\hline $\mathrm{C}$ & 7.8 & 7.1 \\
\hline NM & 5.4 & 6.4 \\
\hline
\end{tabular}

Table 7.2: Density of states (per formula unit) value at the Fermi level of $\mathrm{Sr}_{3} \mathrm{Ru}_{2} \mathrm{O}_{7}$ for various magnetic orders under both LDA and PBE functionals obtained using PAW pseudopotential methods. Note that for the ferromagnetic order (FM), $N\left(E_{F}\right)$ for both spin channels $(\uparrow, \downarrow)$ have been reported. A gaussian broadening 0.0003 Ry was used throughout.

\begin{tabular}{|c|c|c|}
\hline \multirow{2}{*}{ Order } & \multicolumn{2}{|c|}{ PAW-vasp } \\
\hline & LDA & PBE \\
\hline $\mathrm{FM}(\uparrow, \downarrow)$ & $38.6 \quad 23.2$ & $33.6 \quad 33.2$ \\
\hline $\mathrm{E}$ & 47.2 & 30.0 \\
\hline$E^{\prime}$ & 42.6 & 30.6 \\
\hline $\mathrm{A}$ & 36.1 & 35.1 \\
\hline $\mathrm{C}$ & 41.5 & 25.0 \\
\hline NM & 82.4 & 81.6 \\
\hline
\end{tabular}

The individual contributions from each of the three different types of $\mathrm{O}$ atoms are labeled. It can be noted that the $2 p$ contribution from the $\mathrm{O} 3$ atoms, which are the in-plane $\mathrm{O}$, is the largest in the region closest around the Fermi level. It reaffirms the fact that the material is highly two dimensional and most of the electronic transport occurs primarily in-plane. O1 and O2 are respectively the shared and SrO layer apical oxygen atoms and contribute less near the Fermi level. 
In an octahedral crystal field, the $d$ orbitals split into a lower-lying $\mathrm{t}_{2 g}$ manifold, with three bands (and six electrons) and a higher-lying $\mathrm{e}_{g}$ manifold that can accommodate four electrons. The $\mathrm{e}_{g}$ manifold is derived from $\sigma$ antibonding combinations of $\mathrm{O} p$ and $\mathrm{Ru} d$ orbitals, while the normally more narrow $\mathrm{t}_{2 g}$ manifold consists of more weakly antibonding $\pi$ combinations. $\mathrm{Ru}^{4+}$, as in $\mathrm{Sr}_{3} \mathrm{Ru}_{2} \mathrm{O}_{7}$, has four $d$ electrons, which leads to a partially filled $t_{2 g}$ manifold that is responsible for the magnetism and transport. The electronic DOS in the region near the Fermi level is derived from hybridized $\mathrm{Ru}_{2 g}$ and $\mathrm{O} p$ states.

The orbital character is often important in understanding magnetic ordering, especially in systems where transition metal-O hybridization is important, for example double exchange systems [280]. Figure 7.3 shows the projections of $\mathrm{Ru} d$ onto a site with the different magnetic orders as obtained with the PBE functional. As noted previously, non-spin-polarized $\mathrm{Sr}_{3} \mathrm{Ru}_{2} \mathrm{O}_{7}$ has a relatively narrow set of nominally $\mathrm{t}_{2 g}$ orbitals [176]. It should be noted, however, that this crystal-field notation is not strictly correct since the octahedral rotation mixes the $\mathrm{e}_{g}$ and $\mathrm{t}_{2 g}$ manifolds, and the layered structure splits the $t_{2 g}$ orbitals. There is also mixing due to symmetry lowering associated with magnetic order as well as splitting due to interactions between the two layers forming a bilayer.

However, we find that the general shape of the DOS in the energy range of the $t_{2 g}$ orbitals does not depend strongly on magnetic order, showing a higher peak around the Fermi level against a broader peak at $\sim-1 \mathrm{eV}$. The main effect of magnetism 

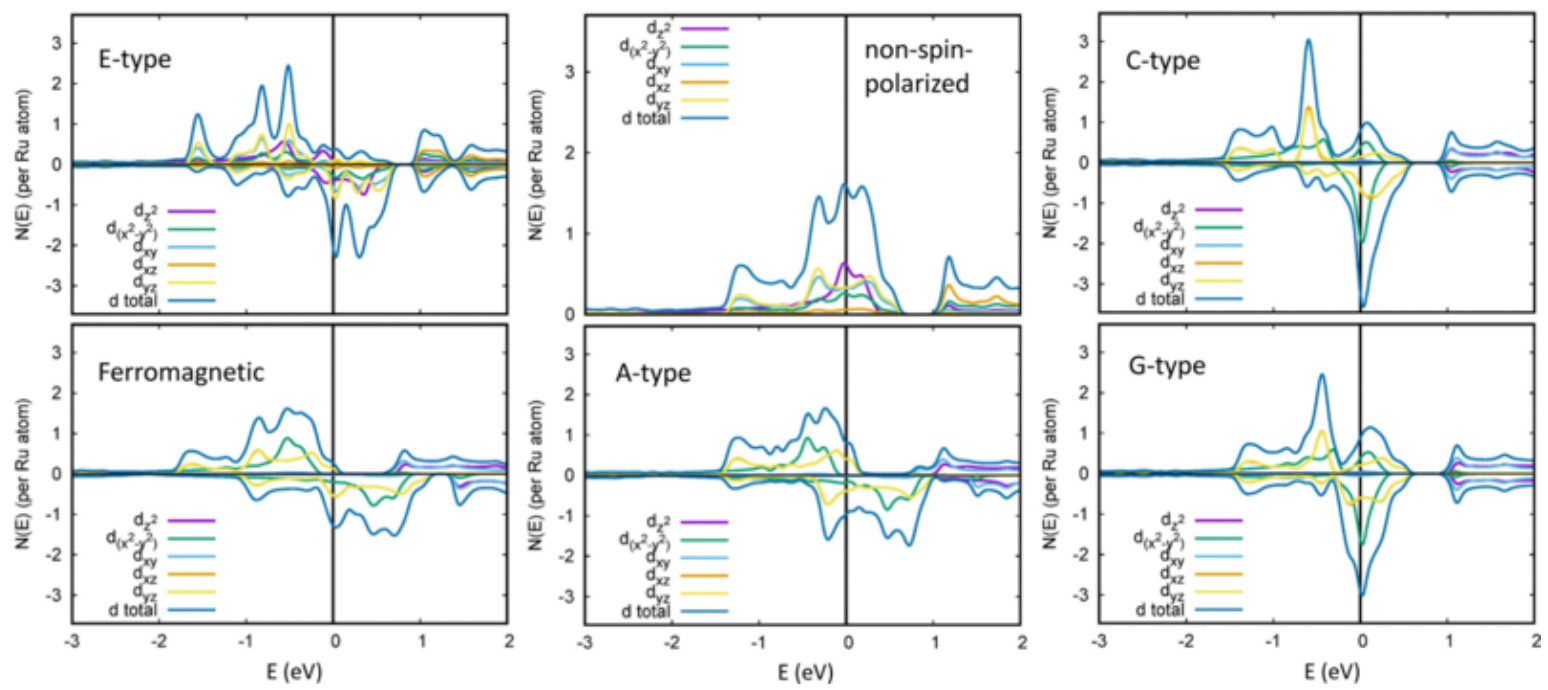

Figure 7.3: Projected DOS of $d$ character on a Ru atom in the energy range of the $\mathrm{t}_{2 g}$ bands for the different magnetic orderings as obtained with the PBE functional. Note that the individual $d$ orbitals are mixed because of the low symmetry induced by the octahedral rotations and magnetic order. The same symmetry was used for the $E$-type and non-spin-polarized. The ferromagnetic, $A$-type, $C$-type, and $G$-type were done in a smaller higher-symmetry cell which leads to a different coordinate system for the $d$ orbitals.

is to exchange split this peak into a lower-lying majority and higher-lying minority components, with the largest exchange splitting for the orders where the moment is highest. The second aspect to note is that the $E$-type order gives a strong narrowing of the individual DOS peaks in the $\mathrm{t}_{2 g}$ manifold. This leads to a greater differentiation of the orbitals. This is also the case for the $C$-type and $G$-type orders, which have nearest-neighbor antiferromagnetism in a single plane. Meanwhile the lowest-energy ferromagnetic and the $A$-type order have generally broader individual peaks. 


\subsection{Electronic transport}

In this section, we calculated the transport using Boltzmann transport theory. In tables 7.3 and 7.4, we show the reduced electrical conductivity $(\sigma / \tau)$ values for different orders obtained using both LDA and PBE functionals. The transport integrals were done for a temperature of $100 \mathrm{~K}$ in the Fermi function for computational convenience. These were calculated using the BoltzTraP code [7]. The BoltzTraP code constructs a smooth interpolation of the energy bands that passes through all the first-principles points. In our calculations we used dense first-principles meshes consisting of $30 \times 16 \times 16$ grids or better so that the interpolated bands are accurate. The BoltzTraP code then does transport integrals using this interpolation to construct the gradients that comprise the band velocities.

Table 7.3: In-plane components of the diagonalized reduced electrical conductivity tensor and the corresponding anisotropies for various magnetic orders with the LDA functional.

\begin{tabular}{cccc}
\hline \hline Order & $\begin{array}{c}\sigma / \tau \\
10^{18}(\Omega m s)^{-1}\end{array}$ & $\begin{array}{c}\text { In-plane } \\
\text { anisotropies }\end{array}$ \\
\hline$E$ & 65 & 77 & 1.17 \\
$E^{\prime}$ & 67 & 75 & 1.13 \\
$A$ & 249 & 250 & 1.00 \\
FM $(\uparrow)$ & 159 & 161 & 1.01 \\
$(\downarrow)$ & 191 & 191 & 1.00 \\
$C$ & 229 & 233 & 1.02 \\
$\mathrm{NM}$ & 266 & 274 & 1.03 \\
\hline \hline
\end{tabular}

To ensure consistency across various magnetic orders, the conductivity tensors have been appropriately diagonalized and only the in-plane directions are given. These are the two largest eigenvalues of the conductivity tensor. In tables 7.3 and 
Table 7.4: In-plane components of the diagonalized reduced electrical conductivity tensor and the corresponding anisotropies for various magnetic orders with the PBE functional.

\begin{tabular}{cccc}
\hline \hline Order & $\begin{array}{c}\sigma / \tau \\
10^{18} \\
(\Omega m s)^{-1}\end{array}$ & $\begin{array}{c}\text { In-plane } \\
\text { anisotropies }\end{array}$ \\
\hline$E$ & 27 & 49 & 1.77 \\
$E^{\prime}$ & 24 & 47 & 1.95 \\
$A$ & 174 & 177 & 1.01 \\
$\mathrm{FM}(\uparrow)$ & 11.4 & 11.5 & 1.01 \\
$(\downarrow)$ & 218 & 222 & 1.02 \\
$C$ & 187 & 189 & 1.01 \\
$G$ & 248 & 256 & 1.03 \\
$\mathrm{NM}$ & 266 & 274 & 1.03 \\
\hline \hline
\end{tabular}

7.4, noticeable anisotropy occurs among the in-plane conductivity components only in the case of the E-type magnetic order. These anisotropy values are noticeably larger than those obtained for any other orders. One may note that within LDA, the in-plane (reduced) electrical conductivity values differ by about $\sim 15 \%$. While it is perhaps not surprising that the $E$-type order gives more anisotropy considering that the pattern of magnetic moments in the $\mathrm{RuO}_{2}$ planes is anisotropic with this order, unlike other simple orders, it is important that this anisotropy in the magnetic pattern is indeed well reflected in the electronic structure at the Fermi level that controls transport. The higher-symmetry (and lower-energy) E-type order is slightly more anisotropic than the $E^{\prime}$ order. In PBE, however, the anisotropies are larger. This reflects its tendency towards larger moments. In this case, when contrasted to LDA, the ordering is reversed and $E^{\prime}$ order has higher anisotropy. 


\subsection{Summary and conclusions}

In the previous two chapters: 6 and 7, we investigated the well-known layered ruthenate $\mathrm{Sr}_{3} \mathrm{Ru}_{2} \mathrm{O}_{7}$. It is considered as one of the best studied examples that display the presence of a quantum critical point. In the presence of a magnetic field, the material shows low-temperature metamagnetic transition. More importantly, using a magnetic field of strength of about $8 \mathrm{~T}$, this critical point transition temperature can be tuned down towards zero kelvin, a signature of quantum criticality. As discussed in chapter 6 , the ground state properties of such a quantum critical material is strongly influenced by strong fluctuations arising from low-energy metastable states of the system. Thus, in order to understand its properties, it becomes imperative to determine these low energy states.

In chapter 6 , we performed a survey of various magnetically ordered states of this ruthenate material that is experimentally known to be paramagnetic. This material when doped with $\mathrm{Mn}$, is reported to display short ranged striped E-type magnetic order [182]. It is interesting to note that our first-principles search indeed predict the striped $E$-type antiferromagnetically ordered state to be the closest competitor to the ground state. It is also worth noting that density functional methods using traditional LDA or PBE functionals are known to overestimate magnetism in itinerant systems lying close to quantum criticality owing to the influence of these strong fluctuations.

In chapter 7, we focus on another novel phenomenon exhibited by layered $\mathrm{Sr}_{3} \mathrm{Ru}_{2} \mathrm{O}_{7}$. In 2007, continued exploration of its properties by Borzi et al. using certain magnetic field revealed that the in-plane electronic transport properties assume certain anisotropy. This anisotropy was found to be increasing with lowering temperature. 
Known as electronic nematicity, it is interesting to note that such an anisotropy is not accompanied by any large structural distortion of the material and is presumably the property of its electron gas itself. The puzzling nature of this phenomenon and its connection to its quantum criticality is not clearly understood. However, our further investigation of this material's transport properties showed us that among all of our investigated magnetic orders, only the striped E-type AFM order, which also is the lowest lying metastable state, is the one which shows an in-plane anisotropy of same order as observed experimentally. This warrants further study of this material, probably using inelastic neutron scattering that can investigate the plausible connection between its quantum criticality and electronic nematicity. 


\section{Chapter 8}

\section{Summary and concluding remarks}

In this thesis, I have discussed the results my investigation on various materials in relation to understand the phenomena of transport and magnetism. I have used all electron density functional based first-principles tools for this purpose. The study of the electronic structure and its interrelation to their individual crystal structure and chemical composition is intended to bring forth the respective microscopic details of these materials in relation to these properties.

For the widely known industrial compound $\mathrm{TiO}_{2}, \mathrm{I}$ found out the energy ordering of two of its polymorphs. One of most accurate all-electron LAPW method is used to investigate whether the long standing discord between the experimental versus theoretical determination of its energy ordering is due to approximations inherent to theoretical methods or some other detail in the experimental factors. I found anatase, in accord with the majority of previous theory studies, to be the ground state of this material, as opposed to experimentally determined rutile. This lead us to conclude 
that either $\mathrm{TiO}_{2}$ is the case which invokes some inherent issue with commonly used density functionals or the experimental methods needs to be revisited to address this conundrum.

I investigated the recently synthesized material $\mathrm{NaSbSe}_{2}$ which was previously known in the literature to be a cation disordered solid. Through the investigation of its electronic structure and thermoelectric transport properties using Boltzmann transport method, I determined that this material bears the properties that are favorable for the developing a thermoelectric (TE). I also used the theoretical metric called the electron fitness function to quantify this and found out that the material indeed has these properties that are at par with other state of art materials used for this purpose. Further experimental studies to realize its bulk form in TE or solar cell purposes would be interesting.

The study of transport continued in chapter 4 where in this instance I centered my discussion around the well-known Lorenz number factor in the context of TE research. I focused on one of the expressions widely popular in the literature for approximating this Lorenz number for experimental TEs. It is used to separate the electronic and lattice components of the thermal conductivity of a TE by using the experimental Seebeck coefficient directly. I performed first-principles calculations on few well-known TE materials alongside the theoretical single parabolic band model to examine the accuracy of this expression. It was found that although for a few TEs like $\mathrm{SrTiO}_{3}$ and the half-Heusler ZrNiSn, the expression works within acceptable margins, the expression gives rise to serious deviations for other practical TEs like $\mathrm{PbTe}$ and $\mathrm{Mg}_{3} \mathrm{Sb}_{2}$. It was also found how this deviation is due to the highly 
non-parabolic electronic structure of these binary TEs where these materials had large deviations over almost their entire doping range within two considered scattering mechanisms. In conclusion to these results, one must exercise caution in using this expression since it is known that high efficiency TEs are especially characterized by their highly non-parabolic band structure, the case where this approximation fails.

In chapter $5, \mathrm{I}$ focussed on magnetism in the perovskite material: $\mathrm{MnSeO}_{3}$. This material is unusual in terms of the chemistry of perovskite. This comes from the fact that this material has $\mathrm{Se}^{4+}$ ions in the $\mathrm{B}$ site whereas the metal $\mathrm{Mn}^{+}$ions occupy the A site. This is enabled by strongly distorted geometry of the structure when compared to the ideal perovskite structure. Our first principles investigation results convey that the material has a ground state magnetic order of G-type antiferromagnetism which agrees with experimental observations. Determination of other magnetic orders and their corresponding energetics lead us to conclude that the material hosts localized magnetic moment such that the on-site moment formation energy is much higher when compared to the inter-site exchange interaction energies. The strong spin-dependent hybridization of suggests analogy of this material with experimentally relevant CMR manganites.

Chapter 6 and 7 are based on the study of two aspects of the material $\mathrm{Sr}_{3} \mathrm{Ru}_{2} \mathrm{O}_{7}$. I discuss the phenomenon of quantum criticality. It is noted how through the manipulation of a non-thermal parameter of the system a quantum critical transition occurs at 0 K. A system near such a criticality is dominated by large fluctuations which in turn determine its ground state properties. $\mathrm{Sr}_{3} \mathrm{Ru}_{2} \mathrm{O}_{7}$ is a known quantum critical material 
that shows metamagnetic transition. Using a magnetic field of about $8 \mathrm{~T}$ strength, this transition temperature can depressed down to $0 \mathrm{~K}$. To understand the nature of these large fluctuations, it is important to know about the low energy metastable states of the system. For $\mathrm{Sr}_{3} \mathrm{Ru}_{2} \mathrm{O}_{7}$, we employed a two fold search strategy to find these lowest energy states. Our first density functional based first principles method yields a ferromagnetic ground state in comparison to an experimental observed paramagnetic order for $\mathrm{Sr}_{3} \mathrm{Ru}_{2} \mathrm{O}_{7}$. This implies the inability of density functionals capture the details of these fluctuations presumably causes the destruction of long range ferromagnetic order. However, owing to the slight orthorhombic distortion of the lattice, we find two striped antiferromagnetic $E$-type ordered state that lies closest in energy to the ground state. The energy ordering of other magnetic states in combination to the sharp peak of density of states at the Fermi level indicates that the magnetism in $\mathrm{Sr}_{3} \mathrm{Ru}_{2} \mathrm{O}_{7}$ is of strong itinerant nature according to the Stoner criteria.

Further, we investigate the nature of this $E$-type order using first-principles based transport calculations and find that these two states display a distinctly large anisotropy among the in-plane conductivity components. This is especially important as $\mathrm{Sr}_{3} \mathrm{Ru}_{2} \mathrm{O}_{7}$ is experimentally known to exhibit "electronic nematicity", i.e. under certain magnetic field Borzi et al. observed a similar conductivity anisotropy. Our results, especially obtaining such an anisotropy of similar order is certainly interesting since the true origin of nematicity property of $\mathrm{Sr}_{3} \mathrm{Ru}_{2} \mathrm{O}_{7}$ is not clearly known. To conclude, inelastic neutron scattering in search of this E-type order would be interesting to determine if this state indeed contributes to the critical fluctuations of this material which in turn would help understand the ground state properties of $\mathrm{Sr}_{3} \mathrm{Ru}_{2} \mathrm{O}_{7}$ better. 
The study of electronic interactions in $\mathrm{Sr}_{3} \mathrm{Ru}_{2} \mathrm{O}_{7}$ is definitely a subject of great interest in order to understand the material better. Closely related to $\mathrm{Sr}_{2} \mathrm{RuO}_{4}$, a known unconventional superconductor, such an understanding will probably shed light on pairing mechanism behind this property. In this spirit, my future research direction is to investigate magnetism in another ruthenate: $\mathrm{SrRuO}_{3}$. This material hosts $\mathrm{Ru}$ in its $\mathrm{V}$ state and forms a honeycomb lattice. The nature of magnetism lies somewhere between localized and itinerant nature. In relation to the recently synthesized $\mathrm{AgRuO}_{3}$, another ruthenate, closely related to this material, my goal would be unravel the nature of electronic interactions, active in these two materials especially magnetism and understand what makes its behaviour so different in relation to other known ruthenates of Strontium. 


\section{Chapter 9}

\section{List of publications}

- A. Putatunda, D.J. Singh, On the ground state of $\mathrm{TiO}_{2}$ as predicted by all electron density functional calculations in relation to experiment, Eur. Phys. J. B 90, $210(2017)$

- C.J. Honer, M.J. Prosniewski, A. Putatunda, D.J. Singh, Properties of the antiferromagnetic selenite $\mathrm{MnSeO}_{3}$ and its non-magnetic analogue $\mathrm{ZnSeO}$ from first principles calculations, J. Phys.: Condens. Matter 29405501 (2017)

- A. Putatunda, G. Xing, J. Sun, Y. Li, D.J. Singh, Thermoelectric properties of layered $\mathrm{NaSbSe}_{2}$, J. Phys.: Condens. Matter 30, 225501 (2018)

- A. Putatunda, D.J. Singh, Lorenz number in relation to estimates based on the Seebeck coefficient, Mater. Today Phys. 8, 49-55 (2019)

- Z. Feng, Y. Fu, A. Putatunda, Y. Zhang, D.J. Singh, Electronic structure as a guide in screening for potential thermoelectrics: Demonstration for half-Heusler 
compounds, Phys. Rev. B 100, 085202 (2019)

- A. Putatunda, G. Qin, W. Ren, D.J. Singh, Competing magnetic orders in quantum critical $\mathrm{Sr}_{3} \mathrm{Ru}_{2} \mathrm{O}_{7}$, Phys. Rev. B 102, 014442 (2020) 


\section{Bibliography}

[1] S. Kim, M. Patel, T.T. Nguyen, J. Yi, C.-P. Wong, J. Kim, Si-embedded metal oxide transparent solar cells, Nano energy 77, 105090 (2020)

[2] Y. Li, D.J. Singh, First principles based screen for identification of transparent conductors, J. Mater. Chem. C 7, 2436 (2019)

[3] E. Snider, N.D.-Gammon, R. McBride, M. Debessai, H. Vindana, K. Vencatasamy, K.V. Lawler, A. Salamat, R.P. Dias, Room-temperature superconductivity in a carbonaceous sulfur hydride, Nature 586, 373-377 (2020)

[4] P. Dai, B.C. Chakoumakos, G.F. Sun, K.W. Wong, Y. Xin, D.F. Lu, Synthesis and neutron powder diffraction study of the superconductor $\mathrm{HgBa}_{2} \mathrm{Ca}_{2} \mathrm{Cu}_{3} \mathrm{O}_{8+\delta}$ by Tl substitution, Physica C: Superconductivity 243, 201-206 (1995)

[5] K. Schwarz, P. Blaha, G.K.H. Madsen, Electronic structure calculations of solids using the WIEN2k package for material sciences, Comput. Phys. Commun. 147, $71(2002)$

[6] D.J. Singh, L. Nordstrom, Planewaves, Pseudopotentials, and the LAPW Method (New York: Springer, 2006) 
[7] G.K.H. Madsen, D.J. Singh, BoltzTraP. A code for calculating band-structure dependent quantities, Comput. Phys. Commun. 175, 67 (2006)

[8] J.P. Heremans, The ugly duckling, Nature 508, 327-328 (2014)

[9] Z. Dughaish, Lead Telluride as a Thermoelectric Material for Thermoelectric Power Generation, Physica B 322, 205-223 (2002)

[10] H.S. Kim, W. Liu, G. Chen, C.-W. Chu, Z. Ren, Relationship between thermoelectric figure of merit and energy conversion efficiency, Proc. Natl. Acad. Sci. 112, 8205-8210 (2015)

[11] G.J. Snyder, E.S. Toberer, Complex thermoelectric materials, Nature Mater. 7, $105(2008)$

[12] P.R. Tulip, Dielectric and Lattice Dynamical Properties of Molecular Crystals via Density Functional Perturbation Theory: Implementation within a First Principles Code, Doctoral Diss., The University of Durham, (2004)

[13] P. Hohenberg, W. Kohn, Inhomogeneous Electron Gas, Phys. Rev. 136, B864 (1964)

[14] D.M. Ceperley, B.J. Alder, Ground State of the Electron Gas by a Stochastic Method, Phys. Rev. Lett. 45, 566 (1980)

[15] J.P. Perdew, Y. Wang, Accurate and simple analytic representation of the electron-gas correlation energy, Phys. Rev. B 45, 13244 (1992)

[16] P.P. Rushton, Towards a Non-Local Density Functional Description of Exchange and Correlation, Doctoral Diss., The University of Durham, (2002) 
[17] J.P. Perdew, K. Burke, M. Ernzerhof, Generalized Gradient Approximation Made Simple, Phys. Rev. Lett. 77, 3865-3868 (1996)

[18] J.P. Perdew, J.A. Chevary, S.H. Vosko, K.A. Jackson, M.R. Pederson, D.J. Singh, C. Fiolhais, Atoms, molecules, solids, and surfaces: Applications of the generalized gradient approximation for exchange and correlation, Phys. Rev. B 46, 6671 (1992)

[19] Z. Wu, R.E. Cohen, More accurate generalized gradient approximation for solids, Phys. Rev. B 73, 235116 (2006)

[20] J.P. Perdew, A. Ruzsinszky, G.I. Csonka, O.A. Vydrov, G.E. Scuseria, L.A. Constantin, X. Zhou, K. Burke, Restoring the Density-Gradient Expansion for Exchange in Solids and Surfaces, Phys. Rev. Lett. 100, 136406 (2008)

[21] J.P. Perdew, A. Zunger, Self-interaction correction to density-functional approximations for many-electron systems, Phys. Rev. B 23, 5048 (1981)

[22] S.H. Vosko, L. Wilk, M. Nusair, Accurate spin-dependent electron liquid correlation energies for local spin density calculations: a critical analysis, Can. J. Phys. $58,1200(1980)$

[23] F. Tran, P. Blaha, Accurate Band Gaps of Semiconductors and Insulators with a Semilocal Exchange-Correlation Potential, Phys. Rev. Lett. 102, 226401 (2009)

[24] G. Kresse, J. Furthmüller, Efficient iterative schemes for ab initio total-energy calculations using a plane-wave basis set, Phys. Rev. B 54, 11169 (1996)

[25] G. Kresse, D. Joubert, From ultrasoft pseudopotentials to the projector augmented-wave method, Phys. Rev. B 59, 1758 (1999) 
[26] J.P. Perdew, Y. Yang, Pair-distribution function and its coupling-constant average for the spin-polarized electron gas, Phys. Rev. B 46, 12947 (1992)

[27] D.S. Sholl, J.A. Steckel, Density functional theory: a practical introduction (John Wiley \& Sons, 2009)

[28] J.C. Slater, Wave functions in a periodic potential, Phys. Rev. 51, 0846-0851 (1937)

[29] C. A.-Draxl, Augmented Planewave Methods, Phys. Scr. 2004, 48 (2004)

[30] R.M. Martin, Electronic structure: basic theory and practical methods, (Cambridge University Press, 2004)

[31] M.R. Ranade, A. Navrotsky, H.Z. Zhang, J.F. Banfield, S.H. Elder, A. Zaban, P.H. Borse, S.K. Kulkarni, G.S. Doran, H.J. Whitfield, Energetics of nanocrystalline $\mathrm{TiO}_{2}$, Proc. Natl. Acad. Sci. USA 99, 6476-6481 (2002)

[32] T. Mitsuhashi, O.J. Kleppa, Transformation Enthalpies of the $\mathrm{TiO}_{2}$ Polymorphs, J. Am. Ceram. Soc. 62, 356-357 (1979)

[33] A. Navrotsky, O.J. Kleppa, Enthalpy of the Anatase-Rutile Transformation, J. Am. Ceram. Soc. 50, 626-626 (1967)

[34] A.A. Levchenko, G. Li, J. Boerio-Goates, B.F. Woodfield, A. Navrotsky, TiO Stability Landscape: Polymorphism, Surface Energy, and Bound Water Energetics, Chem. Mater. 18, 6324-6332 (2006)

[35] A.A. Gribb, J.F. Banfield, Particle size effects on transformation kinetics and phase stability in nanocrystalline $\mathrm{Ti}_{2}$, Am. Mineral. 82, 717-728 (1997) 
[36] N. Satoh, T. Nakashima, K. Yamamoto, Metastability of anatase: size dependent and irreversible anatase-rutile phase transition in atomic-level precise titania, Sci. Rep. 3, 1959 (2013)

[37] P.P. Ahonen, E.I. Kauppinen, J.C. Joubert, J.L. Deschanvres, G. Van Tendeloo, Preparation of nanocrystalline titania powder via aerosol pyrolysis of titanium tetrabutoxide, J. Mater. Res. 14, 3938-3948 (1999)

[38] H. Zhang, J.F. Banfield, Phase transformation of nanocrystalline anatase-torutile via combined interface and surface nucleation, J. Mater. Res. 15, 437-448 (2000)

[39] K. Okada, N. Yamamoto, Y. Kameshima, A. Yasumori, K.J.D. MacKenzie, Effect of Silica Additive on the Anatase-to-Rutile Phase Transition, J. Am. Ceram. Soc. 84, 1591-1596 (2001)

[40] M. Yoshinaka, K. Hirota, O. Yamaguchi, Formation and Sintering of $\mathrm{TiO}_{2}$ (Anatase) Solid Solution in the System $\mathrm{TiO}_{2}-\mathrm{SiO}_{2}$, J. Am. Ceram. Soc. 80, 2749$2753(1997)$

[41] S.J. Smith, R. Stevens, S. Liu, G. Li, A. Navrotsky, J. Boerio-Goates, B.F. Woodfield, Heat capacities and thermodynamic functions of $\mathrm{TiO}_{2}$ anatase and rutile: Analysis of phase stability, Am. Mineral. 94, 236-243 (2009)

[42] M. Mikami, S. Nakamura, O. Kitao, H. Arakawa, X. Gonze, First-Principles Study of Titanium Dioxide: Rutile and Anatase, Jpn. J. Appl. Phys. 39, L847 (2000) 
[43] V. Milman, Properties of complex inorganic solids (Springer US, New York, 1997), pp. 19-24

[44] J. Muscat, V. Swamy, N.M. Harrison, First-principles calculations of the phase stability of $\mathrm{TiO}_{2}$, Phys. Rev. B 65, 224112 (2002)

[45] A. Fahmi, C. Minot, B. Silvi, M. Causá, Theoretical analysis of the structures of titanium dioxide crystals, Phys. Rev. B 47, 11717 (1993)

[46] Z.-H. Cui, F. Wu, H. Jiang, First-principles study of relative stability of rutile and anatase $\mathrm{TiO}_{2}$ using the random phase approximation, Phys. Chem. Chem. Phys. 18, 29914-29922 (2016)

[47] Y. Luo, A. Benali, L. Shulenburger, J.T. Krogel, O. Heinonen, P.R.C. Kent, Phase stability of $\mathrm{TiO}_{2}$ polymorphs from diffusion Quantum Monte Carlo, New J. Phys. 18, 113049 (2016)

[48] J. Trail, B. Monserrat, P. López Ríos, R. Maezono, R.J. Needs, Quantum Monte Carlo study of the energetics of the rutile, anatase, brookite, and columbite $\mathrm{TiO}_{2}$ polymorphs, Phys. Rev. B 95, 121108 (2017)

[49] P. Blaha, D.J. Singh, P.I. Sorantin, K. Schwarz, Electric-field-gradient calculations for systems with large extended-core-state contributions, Phys. Rev. B 46, $1321(1992)$

[50] F. Labat, P. Baranek, C. Domain, C. Minot, C. Adamo, Density functional theory analysis of the structural and electronic properties of $\mathrm{TiO}_{2}$ rutile and anatase polytypes: Performances of different exchange-correlation functionals, J. Chem. Phys. 126, 154703 (2007) 
[51] S.-D. Mo, W.Y. Ching, Electronic and optical properties of three phases of titanium dioxide: Rutile, anatase, and brookite, Phys. Rev. B 51, 13023 (1995)

[52] D.R. Hamann, Generalized Gradient Theory for Silica Phase Transitions, Phys. Rev. Lett. 76, 660 (1996)

[53] C.J. Howard, T.M. Sabine, F. Dickson, Structure and thermal parameters for rutile and anatase, Acta Crystallogr. Sect. B:Struct. Sci. Cryst. Eng. Mater. 47, $462(1991)$

[54] J.K. Burdett, T. Hughbanks, G.J. Miller, J.W. Richardson, J.V. Smith, Structural-Electronic Relationships in Inorganic Solids: Powder Neutron Diffraction Studies of the Rutile and Anatase Polymorphs of Titanium Dioxide at 15 and 295 K, J. Am. Ceram. Soc. 109, 3639 (1987)

[55] D.R. Hamann, Generalized Gradient Theory for Silica Phase Transitions, Phys. Rev. Lett. 76, 660-663 (1996)

[56] X.G. Ma, P. Liang, L. Miao, S.W. Bie, C.K. Zhang, L. Xu, J.J. Jiang, Pressureinduced phase transition and elastic properties of $\mathrm{TiO}_{2}$ polymorphs, Phys. Status Solidi B 246, 2132-2139 (2009)

[57] M. Lazzeri, A. Vittadini, A. Selloni, Structure and energetics of stoichiometric $\mathrm{TiO}_{2}$ anatase surfaces, Phys. Rev. B 63, 155409 (2001)

[58] M. Mohamad, B.U. Haq, R. Ahmed, A. Shaari, N. Ali, R. Hussain, A density functional study of structural, electronic and optical properties of titanium dioxide: Characterization of rutile, anatase and brookite polymorphs, Mater. Sci. Semicond. Process. 31, 405 (2015) 
[59] Y. Jing-Xin, F. Min, J. Guang-Fu, C. Xiang-Rong, Phase transition and thermodynamic properties of $\mathrm{TiO}_{2}$ from first-principles calculations, Chin. Phys. B 18, 269 (2009)

[60] B. Patra, S. Jana, L.A. Constantin, P. Samal, Correct Structural Phase Stability of $\mathrm{FeS}_{2}$, $\mathrm{TiO}_{2}$, and $\mathrm{MnO}_{2}$ from a Semilocal Density Functional, J. Phys. Chem. C 125, 4284-4291 (2021)

[61] J.K. Furdyna, S.-N. Dong, S. Lee, X. Liu, M. Dobrowolska, Chalcogenide From 3D to 2D and Beyond (Woodhead Publishing, 2020), pp. 1-30

[62] B.R. Pamplin, Super-Cell Structure of Semiconductors, Nature 188, 136-137 (1960)

[63] C.H.L. Goodman, The prediction of semiconducting properties in inorganic compounds, J. Phys. Chem. Solids 6, 305-314 (1958)

[64] S. Chen, X.G. Gong, A. Walsh, S.-H. Wei, Electronic structure and stability of quaternary chalcogenide semiconductors derived from cation cross-substitution of II-VI and I-III-VI $I_{2}$ compounds, Phys. Rev. B 79, 165211 (2009)

[65] G. Xing, J. Sun, Y. Li, X. Fan, W. Zheng, D.J. Singh, Electronic fitness function for screening semiconductors as thermoelectric materials, Phys. Rev. Materials $\mathbf{1}$, $065405(2017)$

[66] B.A. Aragaw, J. Sun, D. J. Singh, M.-W. Lee, Ion exchange-prepared NaSbSe 2 nanocrystals: electronic structure and photovoltaic properties of a new solar absorber material, RSC Adv. 7, 45470 (2017) 
[67] C. Wood, Materials for thermoelectric energy conversion, Rep. Prog. Phys. 51, $459(1988)$

[68] J. Yang, H.-L. Yip, A.K.-Y. Jen, Rational Design of Advanced Thermoelectric Materials, Adv. Energy Mater. 3, 549 (2013)

[69] X. Zhang, L.-D. Zhao, Thermoelectric materials: Energy conversion between heat and electricity, J. Materiomics 1, 92 (2015)

[70] V.A. Bazakutsa, N.I. Gnidash, A.K. Kul'chitskaya, A.V. Salov, Photoelectric and optical properties of thin films of ternary chalcogenides of the form $\mathrm{Me}^{I} \mathrm{Sb} \mathrm{X}_{2}^{V I}$, Russ. Phys. J. 18, 472 (1975)

[71] V.A. Bazakutsa, M.P. Vasil'eva, Thermal conductivity of triple semiconductors of $A^{1} S b C_{2}^{6}$ type as a function of chemical composition and structure, J. Eng. Phys. 34, 137 (1978)

[72] J. Sun, D.J. Singh, Electronic Properties, Screening, and Efficient Carrier Transport in $\mathrm{NaSbS}_{2}$, Phys. Rev. Applied 7, 024015 (2017)

[73] S.U. Rahayu, C.-L. Chou, N. Suriyawong, B.A. Aragaw, J.-B. Shi, M.-W. Lee, Sodium antimony sulfide $\left(\mathrm{NaSbS}_{2}\right)$ : Turning an unexpected impurity into a promising, environmentally friendly novel solar absorber material, APL Mater. 4, $116103(2016)$

[74] D.J. Singh, Electronic structure calculations with the Tran-Blaha modified BeckeJohnson density functional, Phys. Rev. B 82, 205102 (2010)

[75] D. Koller, F. Tran, P. Blaha, Merits and limits of the modified Becke-Johnson exchange potential, Phys. Rev. B 83, 195134 (2011) 
[76] Y.-S. Kim, M. Marsman, G. Kresse, F. Tran, P. Blaha, Towards efficient band structure and effective mass calculations for III-V direct band-gap semiconductors, Phys. Rev. B 82, 205212 (2010)

[77] M. Gajdoš, K. Hummer, G. Kresse, J. Furthmüller, F. Bechstedt, Linear optical properties in the projector-augmented wave methodology, Phys. Rev. B 73, 045112 (2006)

[78] B. Eisenmann, H. Schäfer, About seleno- and telluroarsenites, -antimonites and -bismutites, Z. Anorg. Allg. Chem. 456, 87 (1979)

[79] K. Hoang, S.D. Mahanti, Atomic and electronic structures of $I-V-V I_{2}$ ternary chalcogenides, J. Sci.: Adv. Mater. Dev. 1, 51 (2016)

[80] V.I. Bilozertseva, H.M. Khlyap, P.S. Shkumbatyuk, N.L. Dyakonenko, S.S. Krivonos, Sb-contained thin films Structural and electric properties, Mater. Sci.Pol. 24, 929 (2006)

[81] R.E. Cohen, Origin of ferroelectricity in perovskite oxides, Nature 358, 136 (1992)

[82] D. Chattopadhyay, H. J. Queisser, Electron scattering by ionized impurities in semiconductors, Rev. Mod. Phys. 53, 745 (1981)

[83] P. Ghosez, First-principles study of the dielectric and dynamical properties of barium titanate, Doctoral diss., Université Catholique de Louvain (1997)

[84] M.-H. Du, D.J. Singh, Enhanced Born charges in III-VII, IV-VII 2 , and V-VII 3 compounds, Phys. Rev. B 82, 045203 (2010) 
[85] M.D. Neilsen, V. Ozolins, J.P. Heremans, Lone pair electrons minimize lattice thermal conductivity, Energy Environ. Sci. 6, 570 (2013)

[86] D.S. Parker, A.F. May, D.J. Singh, Benefits of Carrier-Pocket Anisotropy to Thermoelectric Performance: The Case of p-type AgBiSe $e_{2}$, Phys. Rev. Applied $\mathbf{3}$, $064003(2015)$

[87] X. Chen, D. Parker, D.J. Singh, Importance of non-parabolic band effects in the thermoelectric properties of semiconductors, Sci. Rep. 3, 3168 (2013)

[88] N.A. Mecholsky, L. Resca, I.L. Pegg, M. Fornari, Theory of band warping and its effects on thermoelectronic transport properties, Phys. Rev. B 89, 155131 (2014)

[89] K. Kuroki, R. Arita, "Pudding Mold" Band Drives Large Thermopower in $\mathrm{Na}_{x} \mathrm{CoO}_{2}$, J. Phys. Soc. Japan 76, 083707 (2007)

[90] D. Parker, X. Chen, D.J. Singh, High Three-Dimensional Thermoelectric Performance from Low-Dimensional Bands, Phys. Rev. Lett. 110, 146601 (2013)

[91] D.I. Bilc, G. Hautier, D. Waroquiers, G.-M. Rignanese, P. Ghosez, LowDimensional Transport and Large Thermoelectric Power Factors in Bulk Semiconductors by Band Engineering of Highly Directional Electronic States, Phys. Rev. Lett. 114, 136601 (2015)

[92] G. Xing, J. Sun, K.P. Ong, X. Fan, W. Zheng, D.J. Singh, Perspective: n-type oxide thermoelectrics via visual search strategies, APL Mater. 4, 053201 (2016)

[93] K. Shirai, K. Yamanaka, Mechanism behind the high thermoelectric power factor of SrTiO3 by calculating the transport coefficients, J. Appl. Phys. 113, 053705 (2013) 
[94] L.D. Hicks, M.S. Dresselhaus, Effect of quantum-well structures on the thermoelectric figure of merit, Phys. Rev. B 47, 12727 (1993)

[95] R. Fei, A. Faghina, R. Sokalski, J.A. Yan, C. Lo, L. Yang, Enhanced Thermoelectric Efficiency via Orthogonal Electrical and Thermal Conductances in Phosphorene, Nano Lett. 14, 6393 (2014)

[96] Y. Pei, X. Shi, A. LaLonde, H. Wang, L. Chen, G.J. Snyder, Convergence of electronic bands for high performance bulk thermoelectrics, Nature 473, 66-69 (2011)

[97] W. Lui, X. Tan, K. Yin, H. Liu, X. Tang, J. Shi, Q. Zhang, C. Uher, Convergence of Conduction Bands as a Means of Enhancing Thermoelectric Performance of n-type $M g_{2} S i_{1-x} S n_{x}$ Solid Solutions, Phys. Rev. Lett. 108, 166601 (2012)

[98] C. Jeong, R. Kim, M.S. Lundstrom, On the best bandstructure for thermoelectric performance: A Landauer perspective, J. Appl. Phys. 111, 113707 (2012)

[99] J. Yang, L. Xi, W. Qiu, L. Wu, X. Shi, L. Chen, J. Yang, W. Zhang, C. Uher, D.J. Singh, On the tuning of electrical and thermal transport in thermoelectrics: an integrated theory-experiment perspective, NPJ Comput. Mater. 2, 15015 (2016)

[100] J. Shuai, J. Mao, S. Song, Q. Zhang, G. Chen, Z. Ren, Recent progress and future challenges on thermoelectric Zintl materials, Mater. Today Phys. 1, 74 (2017)

[101] J. Li, Z. Chen, X. Zhang, Y. Sun, J. Yang, Y. Pei, Electronic origin of the high thermoelectric performance of GeTe among the p-type group IV monotellurides, NPG Asia Mater. 9, e353 (2017) 
[102] H. Tamaki, H. K. Sato, T. Kanno, Isotropic Conduction Network and Defect Chemistry in $\mathrm{Mg}_{3+\delta} \mathrm{Sb}_{2}$-Based Layered Zintl Compounds with High Thermoelectric Performance, Adv. Mater. 28, 10182 (2016)

[103] J. Shuai, J. Mao, S. Song, Q. Zhu, J. Sun, Y. Wang, R. He, J. Zhou, G. Chen, D.J. Singh, Z. Ren, Tuning the carrier scattering mechanism to effectively improve the thermoelectric properties, Energy Environ. Sci. 10, 799 (2017)

[104] L.D. Chen, X.Y. Huang, M. Zhou, X. Shi, W.B. Zhang, The high temperature thermoelectric performances of $\mathrm{Zr}_{0.5} \mathrm{Hf}_{0.5} \mathrm{Ni}_{0.8} \mathrm{Pd}_{0.2} \mathrm{Sn}_{0.99} \mathrm{Sb}_{0.01}$ alloy with nanophase inclusions, J. Appl. Phys. 99, 064305 (2006)

[105] C. Uher, H.J. Goldsmid, Separation of the electronic and lattice thermal conductivities in Bismuth crystals, Phys. Status Solidi B 65, 765-772 (1974)

[106] H. Mizuno, S. Mossa, J.-L. Barrat, Beating the amorphous limit in thermal conductivity by superlattices design, Sci. Rep. 5, 14116 (2015)

[107] Y. Kawaharada, K. Kurosaki, M. Uno, S. Yamanaka, Thermoelectric properties of $\mathrm{CoSb}_{3}$, J. Alloy. Comp. 315, 193 (2001)

[108] J. Yang, L. Xi, W. Qiu, L. Wu, X. Shi, L. Chen, J. Yang, W. Zhang, C. Uher, D.J. Singh, On the tuning of electrical and thermal transport in thermoelectrics: an integrated theory-experiment perspective, NPJ Comput. Mater. 2, 15015 (2016)

[109] K.C. Lukas, W.S. Liu, G. Joshi, M. Zebarjadi, M.S. Dresselhaus, Z.F. Ren, G. Chen, C.P. Opeil, Experimental determination of the Lorenz number in $C u_{0.01} B i_{2} T_{2.7} S e_{0.3}$ and $B i_{0.88} S b_{0.12}$, Phys. Rev. B 85, 205410 (2012) 
[110] W. Liu, J. Hu, S. Zhang, M. Deng, C.-G. Han, Y. Liu, New trends, strategies and opportunities in thermoelectric materials: a perspective, Mater. Today Phys. 1, 50-60 (2017)

[111] M. Yao, C. Opeil, S. Wilson, M. Zebarjadi, Experimental determination of phonon thermal conductivity and Lorenz ratio of single-crystal bismuth telluride, MRS Commun. 7, 922-927 (2017)

[112] E. Maciá, Thermal conductivity in complex metallic alloys: beyond WiedemannFranz law, Phys. Rev. B 79, 245112 (2009)

[113] E. Flage-Larsen, Ø. Prytz, The Lorenz function: its properties at optimum thermoelectric figure-of-merit, Appl. Phys. Lett. 9, 202108 (2011)

[114] G.S. Kumar, G. Prasad, R.O. Pohl, Experimental determinations of the Lorenz number, J. Mater. Sci. 28, 4261-4272 (1993)

[115] N. Hinsche, I. Mertig, P. Zahn, Lorenz function of $B i_{2} T e_{3} / S b_{2} T e_{3}$ superlattices, J. Electron. Mater. 42, 1406-1410 (2013)

[116] M. Thesberg, H. Kosina, N. Neophytou, On the Lorenz number of multiband materials, Phys. Rev. B 95, 125206 (2017)

[117] H.-S. Kim, Z.M. Gibbs, Y. Tang, H. Wang, G.J. Snyder, Characterization of Lorenz number with Seebeck coefficient measurement, APL Mater. 3, 041506 (2015)

[118] J. Liu, P. Wang, M. Wang, R. Xu, J. Zhang, J. Liu, D. Li, N. Liang, Y. Du, G. Chen, G. Tang, Achieving high thermoelectric performance with $\mathrm{Pb}$ and $\mathrm{Zn}$ 
codoped polycrystalline SnSe via phase separation and nanostructuring strategies, Nano Energy 53, 683-689 (2018)

[119] L. Zhang, J. Wang, Z. Cheng, Q. Sun, Z. Li, S. Dou, Lead-free SnTe-based thermoelectrics: enhancement of thermoelectric performance by doping with $\mathrm{Gd} / \mathrm{Ag}$, J. Mater. Chem. A 4, 7936-7942 (2016)

[120] S. Welzmiller, F. Fahrnbauer, F. Hennersdorf, S. Dittmann, M. Liebau, C. Fraunhofer, W.G. Zeier, G.J. Snyder, O. Oeckler, Increasing seebeck coefficients and thermoelectric performance of $\mathrm{Sn} / \mathrm{Sb} / \mathrm{Te}$ and $\mathrm{Ge} / \mathrm{Sb} / \mathrm{Te}$ materials by $\mathrm{Cd}$ doping, Adv. Electron. Mater. 1, 1500266 (2015)

[121] T. Grossfeld, A. Sheskin, Y. Gelbstein, Y. Amouyal, Microstructure evolution of Ag-alloyed PbTe-based compounds and implications for thermoelectric performance, Crystals 7, 281 (2017)

[122] J. Zhang, D. Wu, D. He, D. Feng, M. Yin, X. Qin, J. He, Extraordinary thermoelectric performance realized in n-type PbTe through multiphase nanostructure engineering, Adv. Mater. 29, 1703148 (2017)

[123] X. Wang, V. Askarpour, J. Maassen, M. Lundstrom, On the calculation of Lorenz numbers for complex thermoelectric materials, J. Appl. Phys. 123, 055104 $(2018)$

[124] J.M. Ziman, Principles of the Theory of Solids (2 ${ }^{\text {nd }}$ edition, Cambridge University Press, London, England, 1972)

[125] S. Ahmad, S.D. Mahanti, Energy and temperature dependence of relaxation time and Wiedemann-Franz law on PbTe, Phys. Rev. B 81, 165203 (2010) 
[126] R.W. McKinney, P. Gorai, V. Stevanović, E.S. Toberer, Search for new thermoelectric materials with low Lorenz number, J. Mater. Chem. A 5, 17302-17311 (2017)

[127] M. Lundstrom, C. Jeong, Near-Equilibrium Transport: Fundamentals and Applications, (World Scientific Publishing Co., Singapore, 2013)

[128] A.K. Ganguli, Y.U. Kwon, J.D. Corbett, Three novel zintl phases in the magnesium-lanthanum-antimony system based on intergrowth of $\mathrm{LaSb}$ and $\mathrm{Mg}_{3} \mathrm{Sb}_{2}$ slabs, Inorg. Chem. 32, 4354-4359 (1993)

[129] Y. Noda, K. Masumoto, S. Ohba, Y. Saito, K. Toriumi, Y. Iwata, I. Shibuya, Temperature dependence of atomic thermal parameters of lead chalcogenides, PbS, PbSe and PbTe, Acta Crystallogr. C: Struct. Chem. 43, 1443-1445 (1987)

[130] L. Cao, E. Sozontov, J. Zegenhagen, Cubic to tetragonal phase transition of $\mathrm{SrTiO}_{3}$ under epitaxial stress: an X-ray backscattering study, Phys. Status Solidi A 181, 387-404 (2000)

[131] J. Schmitt, Z.M. Gibbs, G.J. Snyder, C. Felser, Resolving the true band gap of ZrNiSn half-Heusler thermoelectric materials, Mater. Horiz. 2, 68-75 (2015)

[132] J. Zhang, L. Song, S.H. Pedersen, H. Yin, L.T. Hung, B.B. Iversen, Discovery of high-performance low-cost n-type $\mathrm{Mg}_{3} \mathrm{Sh}_{2}$-based thermoelectric materials with multi-valley conduction bands, Nat. Commun. 8, 13901 (2017)

[133] E. Witkoske, X. Wang, M. Lundstrom, V. Askarpour, J. Maassen, Thermoelectric band engineering: the role of carrier scattering, J. Appl. Phys. 122, 175102 (2017) 
[134] C.-H. Su, Experimental determination of lattice thermal conductivity and lorenz number as functions of temperature for n-type PbTe, Mater. Today Phys. 5, 58-63 (2018)

[135] D.K. Bhat, U.S. Shenoy, Enhanced thermoelectric performance of bulk tin telluride: synergistic effect of calcium and indium co-doping, Mater. Today Phys. $\mathbf{4}$, $12-18(2018)$

[136] J. Sun, J. Shuai, Z. Ren, D.J. Singh, Computational modelling of the thermoelectric properties of p-type Zintl compound $\mathrm{CaMg}_{2} \mathrm{Bi}_{2}$, Mater. Today Phys. 2, 40-45 (2017)

[137] W.G. Zeier, A. Zevalkink, Z.M. Gibbs, G. Hautier, M.G. Kanatzidis, G.J. Snyder, Thinking like a chemist: intuition in thermoelectric materials, Angew. Chem. Int. Ed. 55, 6826-6841 (2016)

[138] S. Blundell, Magnetism in condensed matter (Oxford University Press, New York, 2001)

[139] J.A. Alonso, M.J. Martínez-Lope, C.d.l. Calle, A. Muñoz, E. Morán, G. Demazeau, High-pressure synthesis and study of the crystal and magnetic structures of the distorted $\mathrm{SeMO}_{3}(\mathrm{M}=\mathrm{Mn}$, Co, Ni, Zn) perovskites, J. Phys.: Conf. Ser. 121, $032004(2008)$

[140] K. Kohn, K. Inoue, O. Horie, S. Akimoto, Crystal chemistry of $\mathrm{MSeO}_{3}$ and $\mathrm{MTeO}_{3}(\mathrm{M}=\mathrm{Mg}, \mathrm{Mn}, \mathrm{Co}, \mathrm{Ni}, \mathrm{Cu}$, and $\mathrm{Zn})$, J. Solid State Chem. 18, 27-37 (1976) 
[141] K. Kohn, S. Akimoto, K. Inoue, K. Asai, O. Horie, Crystal Structure and Magnetic Properties of $\mathrm{MnSeO}_{3}, \mathrm{CoSeO}_{3}, \mathrm{NiSeO}_{3}$ and $\mathrm{CuSeO}_{3}$, J. Phys. Soc. Jpn. 38, 587-587 (1975)

[142] A. Muñoz, J.A. Alonso, M.J. Martínez-Lope, H. Falcón, M. García-Hernández, E. Morán, High-pressure synthesis and study of the crystal and magnetic structure of the distorted $\mathrm{SeNiO}_{3}$ and $\mathrm{SeMnO}_{3}$ perovskites, Dalton Trans. 41, 4936-4943 (2006)

[143] M.R. Michel, Electronic structure study of copper-containing perovskites, Doctoral Diss. UCL (University College London), (2010)

[144] M.A. Peña, J.L.G. Fierro, Chemical Structures and Performance of Perovskite Oxides, Chem. Rev. 101, 1981-2018 (2001)

[145] L. Pauling, The principles determining the structure of complex ionic crystals, J. Am. Chem. Soc. 51 1010-1026 (1929)

[146] G.H. Jonker, J.H.Van Santen, Ferromagnetic compounds of manganese with perovskite structure, Physica 16 337-349 (1950)

[147] J.H. Van Santen, G.H. Jonker, Electrical conductivity of ferromagnetic compounds of manganese with perovskite structure, Physica 16 599-600 (1950)

[148] R. von Helmolt, J. Wecker, B. Holzapfel, L. Schultz, K. Samwer, Giant negative magnetoresistance in perovskitelike $\mathrm{La}_{2 / 3} \mathrm{Ba}_{2 / 3} \mathrm{MnO}_{x}$ ferromagnetic films, Phys. Rev. Lett. 71, 2331 (1993)

[149] M.S. Islam, R.A. Davines, J.D. Gale, Hop, skip or jump? Proton transport in the $\mathrm{CaZrO}_{3}$ perovskite oxide, Chem. Commun. 2001, 661-662 (2001) 
[150] M.W. Lufaso, P.M. Woodward, Prediction of the crystal structures of perovskites using the software program SPuDS, Acta Crystallogr. B 57, 725-738 (2001)

[151] J.B. Goodenough, An interpretation of the magnetic properties of the perovskitetype mixed crystals $L a_{1-x} S r_{x} \mathrm{CoO}_{3-\lambda}$, J Phys. Chem. Solids 6, 287-297 (1958)

[152] J. Kanamori, Superexchange interaction and symmetry properties of electron orbitals, J. Phys. Chem. Solids 10, 87-98 (1959)

[153] E.E. Rodriguez, F. Poineau, A. Llobet, B.J. Kennedy, M. Avdeev, G.J. Thorogood, M.L. Carter, R. Seshadri, D.J. Singh, A.K. Cheetham, High Temperature Magnetic Ordering in the $4 d$ Perovskite $\mathrm{SrTcO}_{3}$, Phys. Rev. Lett. 106, 067201 (2011)

[154] L.M. Sandratskii, P. Bruno, J. Kudrnovský, On-site Coulomb interaction and the magnetism of (GaMn)N and (GaMn)As, Phys. Rev. B 69, 195203 (2004)

[155] P.W. Anderson, New Approach to the Theory of Superexchange Interactions, Phys. Rev. 115, 2 (1959)

[156] C. Zener, Interaction between the d-Shells in the Transition Metals. II. Ferromagnetic Compounds of Manganese with Perovskite Structure, Phys. Rev. 82, 403 (1951)

[157] P.W. Anderson, H. Hasegawa, Considerations on Double Exchange, Phys. Rev. 100, 675 (1955)

[158] W.E. Pickett, D.J. Singh, Electronic structure and half-metallic transport in the $L a_{1-x} \mathrm{Ca}_{x} \mathrm{MnO}_{3}$ system, Phys. Rev. B 53, 1146 (1996) 
[159] A. Koizumi, S. Miyaki, Y. Kakutani, H. Koizumi, N. Hiraoka, K. Makoshi, N. Sakai, K. Hirota, Y. Murakami, Study of the $e_{g}$ Orbitals in the Bilayer Manganite $\mathrm{La}_{2-2 x} \mathrm{Sr}_{1+2 x} \mathrm{Mn}_{2} \mathrm{O}_{7}$ by Using Magnetic Compton-Profile Measurement, Phys. Rev. Lett. 86, 5589 (2001)

[160] M. Bowen, M. Bibes, A. Barthélémy, J.-P. Contour, A. Anane, Y. Lemaitre, A. Fert, Nearly total spin polarization in $\mathrm{La}_{2 / 3} \mathrm{Sr}_{1 / 3} \mathrm{MnO}_{3}$ from tunneling experiments, Appl. Phys. Lett. 82, 233 (2003)

[161] The idea of criticality, The Frontier Science of Emergent Materials, URL: http://www.physics.rutgers.edu/qcritical/frontier3.htm

[162] P. Coleman, A.J. Schofield, Quantum criticality, Nature 433, 226-229 (2005)

[163] S.A. Grigera, R.S. Perry, A.J. Schofield, M. Chiao, S.R. Julian, G.G. Lonzarich, S.I. Ikeda, Y. Maeno, A.J. Mills, A.P. Mackenzie, Magnetic Field-Tuned Quantum Criticality in the Metallic Ruthenate $\mathrm{Sr}_{3} \mathrm{Ru}_{2} \mathrm{O}_{7}$, Science 294, 329-332 (2001)

[164] N.D. Mathur, F.M. Grosche, S.R. Julian, I.R. Walker, D.M. Freye, R.K.W. Haselwimmer, G.G. Lonzarich, Magnetically mediated superconductivity in heavy fermion compounds, Nature 394, 39-43 (1998)

[165] S.A. Grigera, A.P. Mackenzie, A.J. Schofield, S.R. Julian, G.G. Lonzarich, A metamagnetic quantum critical endpoint in $\mathrm{Sr}_{3} \mathrm{Ru}_{2} \mathrm{O}_{7}$

[166] G. Cao, S. McCall, J.E. Crow, Observation of itinerant ferromagnetism in layered $\mathrm{Sr}_{3} \mathrm{Ru}_{2} \mathrm{O}_{7}$ single crystals, Phys. Rev. B 55, R672(R) (1997) 
[167] Q. Huang, J.W. Lynn, R.W. Erwin, J. Jarupatrakorn, R.J. Cava, Oxygen displacements and search for magnetic order in $\mathrm{Sr}_{3} R u_{2} O_{7}$, Phys. Rev. B 58, 8515 (1998)

[168] S.-I. Ikeda, Y. Maeno, S. Nakatsuji, M. Kosaka, Y. Uwatoko, Ground state in $\mathrm{Sr}_{3} \mathrm{Ru}_{2} \mathrm{O}_{7}$ : Fermi liquid close to a ferromagnetic instability, Phys. Rev B 62, R6089(R) (2000)

[169] S.-I. Ikeda, N. Shirakawa, S. Koiwai, A. Uchida, M. Kosaka, Y. Uwatoko, The pressure dependence of magnetization of layered perovskite oxides with $4 \mathrm{~d}$-electron metal, Phys. C Supercond. its Appl. 364-365, 376-365 (2001)

[170] L. Capogna, E.M. Forgan, S.M. Hayden, A. Wildes, J.A. Duffy, A.P. Mackenzie, R.S. Perry, S. Ikeda, Y. Maeno, and S.P. Brown, Observation of two-dimensional spin fluctuations in the bilayer ruthenate $\mathrm{Sr}_{3} \mathrm{Ru}_{2} \mathrm{O}_{7}$ by inelastic neutron scattering, Phys. Rev. B 67, 012504 (2003)

[171] K. Kitagawa, K. Ishida, R.S. Perry, T. Tayama, T. Sakakibara, Y. Maeno, Metamagnetic Quantum Criticality Revealed by ${ }^{17} O-N M R$ in the Itinerant Metamagnet $\mathrm{Sr}_{3} \mathrm{Ru}_{2} \mathrm{O}_{7}$, Phys. Rev. Lett. 95, 127001 (2005)

[172] R.S. Perry, L.M. Galvin, S.A. Grigera, L. Capogna, A.J. Schofield, A.P. Mackenzie, M. Chiao, S.R. Julian, S.I. Ikeda, S. Nakatsuji, Y. Maeno, and C. Pfleiderer, Metamagnetism and Critical Fluctuations in High Quality Single Crystals of the Bilayer Ruthenate $\mathrm{Sr}_{3} R u_{2} \mathrm{O}_{7}$, Phys. Rev. Lett. 86, 2661 (2001) 
[173] S.A. Grigera, R.A. Borzi, A.P. Mackenzie, S.R. Julian, R.S. Perry, and Y. Maeno, Angular dependence of the magnetic susceptibility in the itinerant metamagnet $S_{3} R u_{2} O_{7}$, Phys. Rev. B 67, 214427 (2003)

[174] H. Shaked, J.D. Jorgensen, O. Chmaissem, S. Ikeda, Y.Maeno, Neutron Diffraction Study of the Structural Distortions in $\mathrm{Sr}_{3} \mathrm{Ru}_{2} \mathrm{O}_{7}$, J. Solid State Chem. 154, $361-367$ (2000)

[175] R. Kiyanagi, K. Tsuda, N. Aso, H. Kimura, Y. Noda, Y. Yoshida, S.-I. Ikeda, Y. Uwatoko, Investigation of the Structure of Single Crystal $\mathrm{Sr}_{3} \mathrm{Ru}_{2} \mathrm{O}_{7}$ by Neutron and Convergent Beam Electron Diffractions, J. Phys. Soc. Jpn. 73, 639-642 (2004)

[176] D.J. Singh, I.I. Mazin, Electronic structure and magnetism of $\mathrm{Sr}_{3} \mathrm{Ru}_{2} \mathrm{O}_{7}$, Phys. Rev. B 63, 165101 (2001)

[177] B. Binz, M. Sigrist, Metamagnetism of itinerant electrons in multi-layer ruthenates, Europhys. Lett. 65, 816 (2004)

[178] S.A. Grigera, P. Gegenwart, R.A. Borzi, F. Weickert, A.J. Schofield, R.S. Perry, T. Tayama, T. Sakakibara, Y. Maeno, A.G. Green, A.P. Mackenzie, DisorderSensitive Phase Formation Linked to Metamagnetic Quantum Criticality, Science 306, 1154-1157 (2004)

[179] H.-Y. Kee, Y.B. Kim, Itinerant metamagnetism induced by electronic nematic order, Phys. Rev. B 71, 184402 (2005)

[180] R.A. Borzi, S.A. Grigera, J. Farrell, R.S. Perry, S.J.S. Lister, S.L. Lee, D.A. Tennant, Y. Maeno, A.P. Mackenzie, Formation of a Nematic Fluid at High Fields in $\mathrm{Sr}_{3} \mathrm{Ru}_{2} \mathrm{O}_{7}$, Science 315, 214-217 (2007) 
[181] E. Fradkin, S.A. Kivelson, M.J. Lawler, J.P. Eisenstein, A.P. Mackenzie, Nematic Fermi Fluids in Condensed Matter Physics, Annu. Rev. Condens. Matter Phys. 1, 153-178 (2010)

[182] D. Mesa, F. Ye, S. Chi, J.A. Fernandez-Baca, W. Tian, B. Hu, R. Jin, E.W. Plummer, J. Zhang, Single-bilayer E-type antiferromagnetism in Mn-substituted $\mathrm{Sr}_{3} \mathrm{Ru}_{2} \mathrm{O}_{\mathbf{7}}$ : Neutron scattering study, Phys. Rev. B 85, 180410(R) (2012)

[183] S. Sachdev, Quantum Phase Transitions (2nd edition, Cambridge University Press, 2011)

[184] P. Gegenwart, Q. Si, F. Steglich, Quantum criticality in heavy-fermion metals, Nat. Phys. 4, 186-197 (2008)

[185] Y. Tokiwa, M. Mchalwat, R.S. Perry, P. Gegenwart, Multiple Metamagnetic Quantum Criticality in $\mathrm{Sr}_{3} \mathrm{Ru}_{2} \mathrm{O}_{7}$, Phys. Rev. Lett. 116, 226402 (2016)

[186] K. Iwaya, S. Satow, T. Hanaguri, N. Shannon, Y. Yoshida, S.I. Ikeda, J.P. He, Y. Kaneko, Y. Tokura, T. Yamada, H. Takagi, Local Tunneling Spectroscopy across a Metamagnetic Critical Point in the Bilayer Ruthenate $\mathrm{Sr}_{3} \mathrm{Ru}_{2} \mathrm{O}_{7}$

[187] W. Wu, A. McCollam, S.A. Grigera, R.S. Perry, A.P. Mackenzie, S.R. Julian, Quantum critical metamagnetism of $\mathrm{Sr}_{3} \mathrm{Ru}_{2} \mathrm{O}_{7}$ under hydrostatic pressure, Phys. Rev. B 83, 045106 (2011)

[188] D.J. Singh, Electronic and magnetic properties of the 4d itinerant ferromagnet $\mathrm{SrRuO}_{3}$, J. Appl. Phys. 79, 4818 (1996) 
[189] P.B. Allen, H. Berger, O. Chauvet, L. Forro, T. Jarlborg, A. Junod, B. Revaz, G. Santi, Transport properties, thermodynamic properties, and electronic structure of $\mathrm{SrRuO}_{3}$, Phys. Rev. B 53, 4393 (1996)

[190] Y. Maeno, H. Hashimoto, K. Yoshida, S. Nishizaki, T. Fujita, J.G. Bednorz, F. Lichtenberg, Superconductivity in a layered perovskite without copper, Nature 372, 532-534 (1994)

[191] K. Ishida, H. Mukuda, Y. Kitaoka, K. Asayama, Z.Q. Mao, Y. Mori, Y. Maeno, Spin-triplet superconductivity in $\mathrm{Sr}_{2} \mathrm{RuO}_{4}$ identified by ${ }^{17} \mathrm{O}$ Knight shift, Nature 396, 658-660 (1998)

[192] A. Pustogow, Y. Luo, A. Chronister, Y.-S. Su, D.A. Sokolov, F. Jerzembeck, A.P. Mackenzie, C.W. Hicks, N. Kikugawa, S. Raghu, E.D. Bauer, S.E. Brown, Constraints on the superconducting order parameter in $\mathrm{Sr}_{2} \mathrm{RuO}_{4}$ from oxygen-17 nuclear magnetic resonance

[193] C. Lester, S. Ramos, R.S. Perry, T.P. Croft, R.I. Bewley, T. Guidi, P. Manuel, D.D. Khalyavin, E.M. Forgan, S.M. Hayden, Field-tunable spin-density-wave phases in $\mathrm{Sr}_{3} \mathrm{Ru}_{2} \mathrm{O}_{7}$, Nature Mater. 14, 373-378 (2015)

[194] J.A.N. Bruin, R.A. Borzi, S.A. Grigera, A.W. Rost, R.S. Perry, A. P. Mackenzie, Study of the electronic nematic phase of $\mathrm{Sr}_{3} \mathrm{Ru}_{2} \mathrm{O}_{7}$ with precise control of the applied magnetic field vector, Phys. Rev. B 87, 161106(R) (2013)

[195] C.M. Puetter, J.G. Rau, H.-Y. Kee, Microscopic route to nematicity in $\mathrm{Sr}_{3} \mathrm{Ru}_{2} \mathrm{O}_{7}$, Phys. Rev. B 81, 081105(R) (2000) 
[196] R.J. Cava, H.W. Zandbergen, J.J. Krajewski, W.F. Peck Jr., B. Batlogg, S. Carter, R.M. Fleming, O.Zhou, L.W. Rupp Jr., Sr $2 \mathrm{RuO}_{4} .0 .25 \mathrm{CO}_{2}$ and the Synthesis and Elementary Properties of $\mathrm{Sr}_{3} \mathrm{Ru}_{2} \mathrm{O}_{7}$, J. Solid State Chem. 116, 141 (1995)

[197] S.-I. Ikeda, Y. Maeno, Magnetic properties of bilayered $S r_{3} R u_{2} O_{7}$, Phys. B: Condens. Matter 259-261, 947 (1999)

[198] M. Zhu, Y. Wang, P.G. Li, J.J. Ge, W. Tian, D. Keavney, Z.Q. Mao, X. Ke, Tipping the magnetic instability in paramagnetic $\mathrm{Sr}_{3} \mathrm{Ru}_{2} \mathrm{O}_{7}$ by Fe impurities, Phys. Rev. B 95, 174430 (2017)

[199] R. Mathieu, A. Asamitsu, Y. Kaneko, J.P. He, X.Z. Yu, R. Kumai, Y. Onose, N. Takeshita, T. Arima, H. Takagi, Y. Tokura, Impurity-induced transition to a Mott insulator in $\mathrm{Sr}_{3} \mathrm{Ru}_{2} \mathrm{O}_{7}$, Phys. Rev. B 72, 092404 (2005)

[200] A. Tamai, M.P. Allan, J.F. Mercure, W. Meevasana, R. Dunkel, D.H. Lu, R.S. Perry, A.P. Mackenzie, D.J. Singh, Z.-X. Shen, F. Baumberger, Fermi Surface and van Hove Singularities in the Itinerant Metamagnet $\mathrm{Sr}_{3} \mathrm{Ru}_{2} \mathrm{O}_{7}$, Phys. Rev. Lett. 101, 026407 (2008)

[201] I.I. Mazin, M.D. Johannes, L. Boeri, K. Koepernik, D.J. Singh, Problems with reconciling density functional theory calculations with experiment in ferropnictides, Phys. Rev. B 78, 085104 (2008)

[202] C. Chen, J. Kim, V.B. Nascimento, Z. Diao, J. Teng, B. Hu, G. Li, F. Liu, J. Zhang, R. Jin, E.W. Plummer, Hidden phases revealed at the surface of doublelayered $\operatorname{Sr}_{3}\left(R u_{1-\mathrm{x}} \mathrm{Mn}_{\mathrm{x}}\right)_{2} \mathrm{O}_{7}$, Phys. Rev. B 94, 085420 (2016) 
[203] M.P. Allan, A. Tamai, E. Rozbicki, M.H. Fischer, J. Voss, P.D.C. King, W. Meevasana, S. Thirupathaiah, E. Rienks, J. Fink, D.A. Tennant, R.S. Perry, J.F. Mercure, M.A. Wang, J. Lee, C.J. Fennie, E.A. Kim, M.J. Lawler, K.M. Shen, A.P. Mackenzie, Z.X. Shen, F. Baumberger, Formation of heavy d-electron quasiparticles in $\mathrm{Sr}_{3} \mathrm{Ru}_{2} \mathrm{O}_{7}$, New J. Phys. 15, 063029 (2013)

[204] M.B. Stone, M.D. Lumsden, R. Jin, B.C. Sales, D. Mandrus, S.E. Nagler, Y. Qiu, Temperature-dependent bilayer ferromagnetism in $\mathrm{Sr}_{3} \mathrm{Ru}_{2} \mathrm{O}_{7}$, Phys. Rev. B 73, $174426(2006)$

[205] T. Moriya, Spin Fluctuations in Itinerant Electron Magnetism (Springer, 1985)

[206] R.B. Laughlin, G.G. Lonzarich, P. Monthoux, D. Pines, The quantum criticality conundrum, Adv. Phys. 50, 361 (2001)

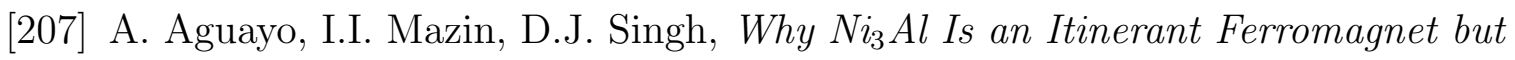
$\mathrm{Ni}_{3} \mathrm{Ga}$ Is Not, Phys. Rev. Lett. 92, 147201 (2004)

[208] P. Larson, I.I Mazin, D.J. Singh, Magnetism, critical fluctuations, and susceptibility renormalization in Pd, Phys. Rev. B 69, 064429 (2004)

[209] I.I. Mazin, D.J. Singh, Spin fluctuations and the magnetic phase diagram of $Z r Z n_{2}$, Phys. Rev. B 69, 020402(R) (2004)

[210] O Gunnarsson, Band model for magnetism of transition metals in the spindensity-functional formalism, J. Phys. F: Met. Phys. 6, 587 (1976)

[211] A.R. Williams, V.L. Moruzzi, C.D. Gelatt Jr., J. Kubler, K. Schwarz, Aspects of transition-metal magnetism, J. Appl. Phys. 53, 2019 (1982) 
[212] Y. Fu, D.J. Singh, Density functional methods for the magnetism of transition metals: SCAN in relation to other functionals, Phys. Rev. B 100, 045126 (2019)

[213] I.I. Mazin, D. J. Singh, Ferromagnetic Spin Fluctuation Induced Superconductivity in $\mathrm{Sr}_{2} \mathrm{RuO}_{4}$, Phys. Rev. Lett. 79, 733 (1997)

[214] W.E. Pickett, Electronic structure of the high-temperature oxide superconductors, Rev. Mod. Phys. 61, 433 (1989)

[215] V.I. Anisimov, F. Aryasetiawan, A.I. Lichtenstein, First-principles calculations of the electronic structure and spectra of strongly correlated systems: the $L D A+U$ method, J. Phys.: Condens. Matter 9, 767 (1997)

[216] S.L. Dudarev, G.A. Botton, S.Y. Savrasov, C.J. Humphreys, A.P. Sutton, Electron-energy-loss spectra and the structural stability of nickel oxide: An LSDA+U study, Phys. Rev. B 57, 1505 (1998)

[217] M. Shimizu, Itinerant electron magnetism, Rep. Prog. Phys. 44, 329 (1981)

[218] , S.N. Kaul, Spin-fluctuation theory for weak itinerant-electron ferromagnets: revisited, J. Phys.: Condens. Matter 11, 7597 (1999)

[219] M.D. Johannes, I.I. Mazin, D.J. Singh, Optical properties and correlation effects in $\mathrm{Na}_{\mathrm{x}} \mathrm{CoO}_{2}$, Phys. Rev. B 71, 205103 (2005)

[220] A. Leithe-Jasper, W. Schnelle, H. Rosner, M. Baenitz, A. Rabis, A.A. Gippius, E.N. Morozova, H. Borrmann, U. Burkhardt, R. Ramlau, U. Schwarz, J.A. Mydosh, Y. Grin, V. Ksenofontov, S. Reiman, Weak itinerant ferromagnetism and electronic and crystal structures of alkali-metal iron antimonides: $\mathrm{NaFe}_{4} \mathrm{Sb}_{12}$ and $K \mathrm{Fe}_{4} \mathrm{Sb}_{12}$, Phys. Rev. B 70, 214418 (2004) 
[221] V.V. Krishnamurthy, J.C. Lang, D. Haskel, D.J. Keavney, G. Srajer, J.L. Robertson, B.C. Sales, D.G. Mandrus, D.J. Singh, and D.I. Bilc, Ferrimagnetism in $\mathrm{EuFe}_{4} \mathrm{Sb}_{12}$ due to the Interplay of $f$-Electron Moments and a Nearly Ferromagnetic Host, Phys. Rev. Lett. 98, 126403 (2007)

[222] D.J. Singh, Evidence for proximity of $\mathrm{YFe}_{2} \mathrm{Si}_{2}$ to a magnetic quantum critical point, Phys. Rev. B 93, 245155 (2016)

[223] D.J. Singh, Quantum critical behavior and possible triplet superconductivity in electron-doped $\mathrm{CoO}_{2}$ sheets, Phys. Rev. B 68, 020503(R) (2003)

[224] Y. Ihara, H. Takeya, K. Ishida, H. Ikeda, C. Michioka, K. Takada, T. Sasaki, H. Sakurai, E. Takayama-Muromachi, Unconventional Superconductivity Induced by Quantum Critical Fluctuations in Hydrate Cobaltate $\mathrm{Na}_{\mathrm{x}}\left(\mathrm{H}_{3} \mathrm{O}\right)_{\mathrm{z}} \mathrm{CoO}_{2} \cdot \mathrm{yH}_{2} \mathrm{O}$ Relationship between Magnetic Fluctuations and Superconductivity Revealed by Co Nuclear Quadrupole Resonance, J. Phys. Soc. Jpn. 75, 124714 (2006)

[225] Y. Ihara, H. Takeya, K. Ishida, C. Michioka, K. Yoshimura, K. Takada, T. Sasaki, H. Sakurai, E. Takayama-Muromachi, Quantum critical behavior in superconducting $\mathrm{Na}_{\mathrm{x}}\left(\mathrm{H}_{3} \mathrm{O}\right)_{\mathrm{z}} \mathrm{CoO}_{2} \cdot y \mathrm{H}_{2} \mathrm{O}$ observed in a high-field Co NMR experiment, Phys. Rev. B 75, 212506 (2007)

[226] D.J. Singh, Superconductivity and magnetism in $\mathrm{YFe}_{2} \mathrm{Ge}_{2}$, Phys. Rev. B 89, $024505(2014)$

[227] N. Sirica, F. Bondino, S. Nappini, I. Pis, L. Poudel, A.D. Christianson, D. Mandrus, D.J. Singh, N. Mannella, Spectroscopic evidence for strong quantum 
spin fluctuations with itinerant character in $\mathrm{YFe}_{2} \mathrm{Ge}_{2}$, Phys. Rev. B 91, 121102(R) (2015)

[228] D. Zhao, H.L. Wo, J. Li, D.W. Song, L.X. Zheng, S.J. Li, L.P. Nie, X.G. Luo, J. Zhao, T. Wu, X. H. Chen, Approaching itinerant magnetic quantum criticality through a Hund's coupling induced electronic crossover in the $\mathrm{YFe}_{2} \mathrm{Ge}_{2}$ superconductor, Phys. Rev. B 101, 064511 (2020)

[229] H. Wo, Q. Wang, Y. Shen, X. Zhang, Y. Hao, Y. Feng, S. Shen, Z. He, B. Pan, W. Wang, K. Nakajima, S. Ohira-Kawamura, P. Steffens, M. Boehm, K. Schmalzl, T.R. Forrest, M. Matsuda, Y. Zhao, J.W. Lynn, Z. Yin, J. Zhao, Coexistence of Ferromagnetic and Stripe-Type Antiferromagnetic Spin Fluctuations in $\mathrm{YFe}_{2} \mathrm{Ge}_{2}$, Phys. Rev. Lett. 122, 217003 (2019)

[230] A. Kanbayashi, Magnetic Properties of New Cubic-Perovskite Mixtures; $\left(L a_{0.5} N a_{0.5}\right) R u O_{3}$ and $\left(C a_{1-\mathrm{x}} A_{\mathrm{x}}\right) R u O_{3} \quad(A=M g, S r)$, J. Phys. Soc. Jpn. 44, 108 (1978)

[231] I.I. Mazin, D.J. Singh, Electronic structure and magnetism in Ru-based perovskites, Phys. Rev. B 56, 2556 (1997)

[232] K. Maiti, R.S. Singh, Evidence against strong correlation in 4 d transition-metal oxides $\mathrm{CaRuO}_{3}$ and $\mathrm{SrRuO}_{3}$, Phys. Rev. B 71, 161102(R) (2005).

[233] N. Miao, N.C. Bristowe, B. Xu, M.J. Verstraete, P. Gosez, First-principles study of the lattice dynamical properties of strontium ruthenate, J. Phys.: Condens. Matter 26, 035401 (2014) 
[234] B. Nadgorny, M.S. Osofsky, D.J. Singh, G.T. Woods, R.J. Soulen Jr., Measurements of spin polarization of epitaxial $\mathrm{SrRuO}_{3}$, Appl. Phys. Lett. 82, 427 (2003)

[235] P. Raychaudhuri, A.P. Mackenzie, J.W. Reiner, M.R. Beasley, Transport spin polarization in $\mathrm{SrRuO}_{3}$ measured through point-contact Andreev reflection, Phys. Rev. B 67, 020411(R) (2003)

[236] D.C. Worledge, T.H. Geballe, Negative Spin-Polarization of $\mathrm{SrRuO}_{3}$, Phys. Rev. Lett. 85, $5182(2000)$

[237] J.M. Rondinelli, N.M. Caffrey, S. Sanvito, N.A. Spaldin, Electronic properties of bulk and thin film SrRuO$O_{3}$ : Search for the metal-insulator transition, Phys. Rev. B 78, 155107 (2008)

[238] A.T. Zayak, X. Huang, J.B. Neaton, K. M. Rabe, Structural, electronic, and magnetic properties of $\mathrm{SrRuO}_{3}$ under epitaxial strain, Phys. Rev. B 74, 094104 (2006)

[239] G. Cao, O. Korneta, S. Chikara, L.E. DeLong, P. Schlottmann, Non-Fermiliquid behavior in single-crystal $\mathrm{CaRuO}_{3}$ : Comparison to ferromagnetic $\mathrm{SrRuO}$, Solid State Commun. 148, 305 (2008)

[240] N. Kikugawa, L. Balicas, A.P. Mackenzie, Physical Properties of SingleCrystalline $\mathrm{CaRuO}_{3}$ Grown by a Floating-Zone Method, J. Phys. Soc. Jpn. 78, $014701(2009)$

[241] Z.V. Pchelkina, I.A. Nekrasov, T. Pruschke, A. Sekiyama, S. Suga, V.I. Anisimov, D. Vollhardt, Evidence for strong electronic correlations in the spectra of $\mathrm{Sr}_{2} \mathrm{RuO}_{4}$, Phys. Rev. B 75, 035122 (2007) 
[242] D.J. Singh, Comment on "Evidence for strong electronic correlations in the spectra of $\mathrm{Sr}_{2} \mathrm{RuO}_{4}$ ”, Phys. Rev. B 77, 046101 (2008)

[243] N.J.C. Ingle, K.M. Shen, F. Baumberger, W. Meevasana, D.H. Lu, Z.-X. Shen, S. Nakatsuji, Z.Q. Mao, Y. Maeno, T. Kimura, Y. Tokura, Quantitative analysis of $\mathrm{Sr}_{2} \mathrm{RuO}_{4}$ angle-resolved photoemission spectra: Many-body interactions in a model Fermi liquid, Phys. Rev. B 72, 205114 (2005).

[244] A. Damascelli, D.H. Lu, K.M. Shen, N.P. Armitage, F. Ronning, D.L. Feng, C. Kim, Z.X. Shen, T. Kimura, Y. Tokura, Z.Q. Mao, Y. Maeno, Fermi Surface, Surface States, and Surface Reconstruction in $\mathrm{Sr}_{2} R u \mathrm{O}_{4}$, Phys. Rev. Lett. 85, 5194 (2000)

[245] T. Oguchi, Electronic band structure of the superconductor $\mathrm{Sr}_{2} \mathrm{RuO}_{4}$, Phys. Rev. B 51, 1385(R) (1995)

[246] D.J. Singh, Relationship of $\mathrm{Sr}_{2} \mathrm{RuO}_{4}$ to the superconducting layered cuprates, Phys. Rev. B 52, 1358 (1995)

[247] T. Katsufuji, M. Kasai, Y. Tokura, In-Plane and Out-of-Plane Optical Spectra of $\mathrm{Sr}_{2} \mathrm{RuO}_{4}$, Phys. Rev. Lett. 76, 126 (1996)

[248] C. Bergemann, S.R. Julian, A.P. Mackenzie, S. NishiZaki, Y. Maeno, Detailed Topography of the Fermi Surface of $\mathrm{Sr}_{2} \mathrm{RuO}_{4}$, Phys. Rev. Lett. 84, 2662 (2000)

[249] M. Sigrist, D. Agterberg, T.M. Rice, M.E. Zhitomirsky, $\mathrm{Sr}_{2} \mathrm{RuO}_{4}-\mathrm{A}$ realization of spin triplet pairing?, Physica C 282-287, 214 (1997) 
[250] I.I. Mazin, D. J. Singh, Competitions in Layered Ruthenates: Ferromagnetism versus Antiferromagnetism and Triplet versus Singlet Pairing, Phys. Rev. Lett. 82, $4324(1999)$

[251] M. Braden, Y. Sidis, P. Bourges, P. Pfeuty, J. Kulda, Z. Mao, Y. Maeno, Inelastic neutron scattering study of magnetic excitations in $\mathrm{Sr}_{2} \mathrm{RuO}_{4}$, Phys. Rev. B 66, $064522(2002)$

[252] S. Nakatsuji, S.I. Ikeda, Y. Maeno, $\mathrm{Ca}_{2} \mathrm{RuO}_{4}$ : New Mott Insulators of Layered Ruthenate, J. Phys. Soc. Jpn. 66, 1868 (1997)

[253] J.P. Carlo, T. Goko, I.M. Gat-Malureanu, P.R. Russo, A.T. Savici, A.A. Aczel, G.J. MacDougall, J.A. Rodriguez, T.J. Williams, G.M. Luke, C.R. Wiebe, Y. Yoshida, S. Nakatsuji, Y. Maeno, T. Taniguchi, Y. J. Uemura, New magnetic phase diagram of $(\mathrm{Sr}, \mathrm{Ca})_{2} \mathrm{Ru} \mathrm{O}_{4}$, Nature Mater. 11, 323 (2012)

[254] R.S. Perry, L.M. Galvin, A.P. Mackenzie, D.M. Forsythe, S.R. Julian, S.I. Ikeda, Y. Maeno, Hall effect of $\mathrm{Sr}_{3} R u_{2} \mathrm{O}_{7}$, Physica B: Condens. Matter 284-288, 1469 (2000)

[255] S.-I. Ikeda, N. Shirakawa, T. Yanagisawa, Y. Yoshida, S. Koikegami, S. Koike, M. Kosaka, Y. Uwatoko, Uniaxial-Pressure Induced Ferromagnetism of Enhanced Paramagnetic $\mathrm{Sr}_{3} \mathrm{Ru}_{2} \mathrm{O}_{7}$, J. Phys. Soc. Jpn. 73, 1322-1325 (2004)

[256] B. Hu, G.T. McCandless, M. Menard, V.B. Nascimento, J.Y. Chan, E.W. Plummer, R. Jin, Surface and bulk structural properties of single-crystalline $\mathrm{Sr}_{3} \mathrm{Ru}_{2} \mathrm{O}_{7}$, Phys. Rev. B 81, 184104 (2010) 
[257] J. Hooper, M.H. Fang, M. Zhou, D. Fobes, N. Dang, Z.Q. Mao, C.M. Feng, Z.A. Xu, M.H. Yu, C.J. O'Connor, G.J. Xu, N. Andersen, M. Salamon, Competing magnetic fluctuations in $\mathrm{Sr}_{3} R u_{2} \mathrm{O}_{7}$ probed by Ti doping, Phys. Rev. B 75, 060403(R) (2007)

[258] C. Piefke, F. Lechermann, LDA + slave boson approach to the correlated electronic structure of the metamagnetic bilayer ruthenate $\mathrm{Sr}_{3} \mathrm{Ru}_{2} \mathrm{O}_{7}$, Phys. Status Solidi B 248, 2269-2275 (2011)

[259] M. Behrmann, C. Piefke, F. Lechermann, Multiorbital physics in Fermi liquids prone to magnetic order, Phys. Rev. B 86, 045130 (2012)

[260] C. Autieri, M. Cuoco, C. Noce, Structural and electronic properties of $\mathrm{Sr}_{2} R u \mathrm{O}_{4} / \mathrm{Sr}_{3} R u_{2} \mathrm{O}_{7}$ heterostructures, Phys. Rev. B 89, 075102 (2014)

[261] S. Mukherjee, W.-C. Lee, Structural and magnetic field effects on spin fluctuations in $\mathrm{Sr}_{3} \mathrm{Ru}_{2} \mathrm{O}_{7}$, Phys. Rev. B 94, 064407 (2016)

[262] P. Rivero, V. Meunier, W. Shelton, Revealing out-of-equilibrium hidden phases in $\mathrm{Sr}_{3} R u_{2} \mathrm{O}_{7}$ by applying stress, Phys. Rev. B 97, 134116 (2018)

[263] P. Rivero, R. Jin, C. Chen, V. Meunier, E. Plummer, W. Shelton, Predicting hidden bulk phases from surface phases in bilayered $\mathrm{Sr}_{3} \mathrm{Ru}_{2} \mathrm{O}_{7}$, Sci. Rep. 7, 10265 (2017)

[264] P. Steffens, J. Farrell, S. Price, A.P. Mackenzie, Y. Sidis, K. Schmalzl, M. Braden, ncommensurate magnetic ordering in Ti-doped $\mathrm{Sr}_{3} R u_{2} \mathrm{O}_{7}$, Phys. Rev. B 79, $054422(2009)$ 
[265] I. Hase, Y. Nishihara, Electronic Structure of $\mathrm{Sr}_{3} R u_{2} \mathrm{O}_{7}$, J. Phys. Soc. Jpn. 66, $3517(1997)$

[266] W.-C. Lee, D.P. Arovas, C. Wu, Quasiparticle interference in the unconventional metamagnetic compound $\mathrm{Sr}_{3} R u_{2} \mathrm{O}_{7}$, Phys. Rev. B 81, 184403 (2010)

[267] M. Cococcioni, S. de Gironcoli, Linear response approach to the calculation of the effective interaction parameters in the $L D A+U$ method, Phys. Rev. B 71, $035105(2005)$

[268] M.A. Hossain, I. Zegkinoglou, Y.-D. Chuang, J. Geck, B. Bohnenbuck, A.G.C. Gonzalez, H.-H. Wu, C. Schüßler-Langeheine, D.G. Hawthorn, J.D. Denlinger, R. Mathieu, Y. Tokura, S. Satow, H. Takagi, Y. Yoshida, Z. Hussain, B. Keimer, G.A. Sawatzky, A. Damascelli, Electronic superlattice revealed by resonant scattering from random impurities in $\mathrm{Sr}_{3} \mathrm{Ru}_{2} \mathrm{O}_{7}$, Sci. Rep. 3, 2299 (2013)

[269] M.A. Hossain, Z. Hu, M.W. Haverkort, T. Burnus, C.F. Chang, S. Klein, J.D. Denlinger, H.-J. Lin, C.T. Chen, R. Mathieu, Y. Kaneko, Y. Tokura, S. Satow, Y. Yoshida, H. Takagi, A. Tanaka, I.S. Elfimov, G.A. Sawatzky, L.H. Tjeng, A. Damascelli, Crystal-Field Level Inversion in Lightly Mn-Doped $\mathrm{Sr}_{3} \mathrm{Ru}_{2} \mathrm{O}_{7}$, Phys. Rev. Lett. 101, 016404 (2008)

[270] G. Li, Q. Li, M. Pan, B. Hu, C. Chen, J. Teng, Z. Diao, J. Zhang, R. Jin, E.W. Plummer, Atomic-Scale Fingerprint of Mn Dopant at the Surface of $\mathrm{Sr}_{3}\left(R u_{1-\mathrm{x}} \mathrm{Mn}_{\mathrm{x}}\right)_{2} \mathrm{O}_{7}$, Sci. Rep. 3, 2882 (2013) 
[271] B. Hu, G.T. McCandless, V.O. Garlea, S. Stadler, Y. Xiong, J.Y. Chan, E.W. Plummer, R. Jin, Structure-property coupling in $\operatorname{Sr}_{3}\left(R u_{1-\mathrm{x}} \mathrm{Mn}_{\mathrm{x}}\right)_{2} \mathrm{O}_{7}$, Phys. Rev. B 84, $174411(2011)$

[272] C. Autieri, Antiferromagnetic and xy ferro-orbital order in insulating $\mathrm{SrRuO}_{3}$ thin films with SrO termination, J. Phys.: Condens. Matter 28, 426004 (2016)

[273] J. Leshen, M. Kavai, I. Giannakis, Y. Kaneko, Y. Tokura, S. Mukherjee, W.-C. Lee, P. Aynajian, Emergent charge order near the doping-induced Mott-insulating quantum phase transition in $\mathrm{Sr}_{3} \mathrm{Ru}_{2} \mathrm{O}_{7}$, Commun. Phys. 2, 36 (2019)

[274] P. Rivero, V. Meunier, W. Shelton, Half-metallic ferromagnetism in $\mathrm{Sr}_{3} \mathrm{Ru}_{2} \mathrm{O}_{7}$, Phys. Rev. B 95, 195106 (2017)

[275] D.O. Brodsky, M.E. Barber, J.A.N. Bruin, R.A. Borzi, S.A. Grigera, R.S. Perry, A.P. Mackenzie, C.W. Hicks, Strain and vector magnetic field tuning of the anomalous phase in $\mathrm{Sr}_{3} \mathrm{Ru}_{2} \mathrm{O}_{7}$, Sci. Adv. 3, e1501804 (2017)

[276] P.B. Marshall, K. Ahadi, H. Kim, S. Stemmer, Electron nematic fluid in a strained $\mathrm{Sr}_{3} \mathrm{Ru}_{2} \mathrm{O}_{7}$ flim, Phys. Rev. B 97, 155160 (2018)

[277] A. Subedi, D.J. Singh, Band structure and itinerant magnetism in quantum critical $\mathrm{NbFe}_{2}$, Phys. Rev. B 81, 024422 (2010)

[278] D.J. Singh, Prospects for quantum criticality in perovskite $\operatorname{SrRhO}_{3}$, Phys. Rev. B 67, 054507 (2003)

[279] D.J. Singh, Electronic structure and weak itinerant magnetism in metallic $Y_{2} N i_{7}$, Phys. Rev. B 92, 174403 (2015) 
[280] W. Brzezicki, C. Noce, A. Romano, M. Cuoco, Zigzag and Checkerboard Magnetic Patterns in Orbitally Directional Double-Exchange Systems, Phys. Rev. Lett. 114, $247002(2015)$

[281] A.P. Mackenzie, J.A.N. Bruin, R.A. Borzi, A.W. Rost, S.A. Grigera, Quantum criticality and the formation of a putative electronic liquid crystal in $\mathrm{Sr}_{3} \mathrm{Ru}_{2} \mathrm{O}_{7}$, Physica C 481, 207-214 (2012)

[282] C. Stingl, R.S. Perry, Y. Maeno, P. Gegenwart, Electronic nematicity and its relationto quantum criticality in $\mathrm{Sr}_{3} \mathrm{Ru}_{2} \mathrm{O}_{7}$ studied by thermal expansion, Phys. Status Solidi B 250, 450-456 (2013) 


\section{VITA}

I was born in the suburbs of Kolkata, the capital city of West Bengal state of India. My family moved to Kanpur in Northern India where I grew up and finished my schooling. Here I first developed my interest in Physics. I went on obtain my integrated masters degree in Physics from National institute of Science Education and Research where I was first introduced to research and got to know about various advanced topics. During these years I developed a knack for computational physics. Upon graduating, Dr. David Singh offered me to work with him for my doctoral thesis. In 2016, I moved to Columbia, Missouri to start my PhD journey. I was able to start my research work in my first year under Dr. Singh's guidance. Upon receiving research assistantship, I continued my work on electronic structure of materials with his valuable financial aid. I also taught few undergraduate laboratory courses to gain teaching experience. I expect to graduate with my doctoral degree in 2021. 